\title{
Flexibility Envelopes For Power System Operational Planning
}

\author{
Hussam Nosair

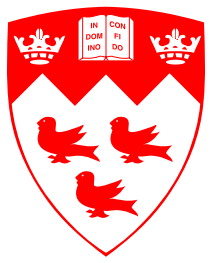 \\ Department of Electrical \& Computer Engineering \\ McGill University \\ Montreal, Canada
}

December 2015

A thesis submitted to McGill University in partial fulfillment of the requirements for the degree of Doctor of Philosophy.

(c) 2015 Hussam Nosair 


\begin{abstract}
Operational planning is an integral part of maintaining economic operation of the bulk power system and keeping the lights on. Traditionally, power systems have been designed without ramping capability concerns, such that operational planning needed only focus on capacity adequacy, while assuming sufficient ramping capability. Today, the integration of renewable energy sources has led to increased variability and uncertainty in the power balance, thus warranting the need to carefully assess the ramping adequacy of resources on top of capacity requirements. Moreover, the advent of energy-constrained resources, such as energy storage and demand response, has warranted the need to plan power systems in the face of energy scarcity, in addition to ramping and capacity scarcity. Consequently, traditional operational planning is becoming less capable of handling the new reality of modern sustainable power systems integrating high levels of renewable energy. This has led to the emerging concept of power system flexibility, to emphasize on the need to plan capacity, ramping, and energy altogether. Concurrently, there has also been an increasing interest in stochastic planning to handle the increased uncertainty. That being said, the current state of the art on operational planning remains confined to the traditional thinking, especially when defining operating reserve requirements. To that end, this dissertation proposes a modernization of the concept of operating reserve, in light of the emerging concept of power system flexibility. The concept of a flexibility requirement envelope is introduced to accurately capture the capacity, ramping, and energy requirements arising from variability and uncertainty. Equally, the concept of a flexibility envelope is proposed to capture the capacity, ramping, and energy capability of a power system resource. Adequate system flexibility is asserted when the aggregate flexibility envelope of power system resources encloses the flexibility requirement envelope while performing operational planning. The concept of envelope enclosure is formulated both deterministically and probabilistically, and its effectiveness is illustrated via several examples throughout the dissertation. Furthermore, the dissertation proposes dynamical operational planning, which is expected to open new avenues in operational planning research and practice.
\end{abstract}




\section{Résumé}

La planification opérationnelle fait partie intégrante du maintien d'un régime opérationnel économique et fiable des réseaux électriques. Traditionnellement, les réseaux ont été conçus sans avoir à se soucier des phénomènes de rampes de puissance, de telle sorte que la planification opérationnelle devait uniquement traiter l'adéquation des capacités en partant du principe que les capacités de rampe des équipements du réseau étaient suffisantes. De nos jours, l'intégration des sources d'énergie renouvelables a contribué à augmenter la variabilité et l'incertitude dans l'équilibre de puissance entre la production et la charge. Cela nécessite d'évaluer précisément l'adéquation des capacités de rampe et de puissance des ressouces du réseau. De plus, l'arrivée de ressources contraignantes en termes d'énergie, que ce soit par rapport au stockage de l'énergie ou au pilotage de charge, rend nécessaire la planification de systèmes électriques capables de faire face à la raréfaction de l'énergie, de puissance disponible et de rampe. Par conséquent, la planification opérationnelle traditionnelle devient moins apte à gérer la nouvelle réalité des systèmes électriques durables modernes qui intègrent fortement les énergies renouvelables. Ceci a conduit à l'émergence du concept de flexibilité du système électrique, soulignant l'importance d'une planification conjointe de la puissance disponible, des rampes et de l'énergie. En parallèle, on observe également un intérêt croissant pour la planification stochastique afin de gérer l'incertitude croissante. Cela étant, l'état actuel de la technique concernant la planification opérationnelle reste confiné à la pensée traditionnelle, et notamment lorsqu'il s'agit de définir les contraintes de réserves d'exploitation. A cette fin, ce mémoire propose de moderniser le concept de réserve d'exploitation à la lumière du concept émergeant de la flexibilité. Le concept d'une enveloppe des exigences de flexibilité est proposé pour capter le besoin en puissance disponible, en rampe et énergie qui découlent de la variabilité et de l'incertitude mentionnées plus haut. De la même manière, le concept d'une enveloppe de flexibilité est développé pour déterminer les moyens en termes de puissance, en rampe et en énergie d'une ressource d'un système électrique. Une flexibilité de système adéquate est appliquée, lorsque l'enveloppe de flexibilité totale des ressources du système électrique comprend l'enveloppe des exigences de flexibilité, lors de la planification opérationnelle. Le concept d'enveloppe est formulé à la fois via une approche déterministe et via une approche probabiliste. Son efficacité est illustrée grâce à plusieurs exemples dans cette thèse. En outre, cette thèse évoque une planification opérationnelle dynamique, qui devrait ouvrir de 
nouvelles voies dans le domaine de la planification opérationnelle et faire avancer l'état de la technique. 


\section{Acknowledgments}

This dissertation has been made possible with the financial support of the McGill Engineering Doctoral Awards (MEDA) and the Natural Sciences and Engineering Research Council of Canada (NSERC).

Furthermore, I would like to extend my warmest gratitude and appreciation to my $\mathrm{PhD}$ advisor, Prof. Francois Bouffard, for his continuous support and mentorship throughout the four years of PhD studies.

Moreover, I would like to sincerely thank the thesis advisory committee, consisting of Prof. Ioannis Psaromiligkos and Prof. Aditya Mahajan, for their advice and supervisory roles throughout the PhD program.

Overall, I've had a pleasant experience being part of McGill University which happens to be a renowned institution in the eye of the world. 


\section{Contents}

1 Introduction $\quad 1$

1.1 The North American Bulk Power System in Transition . . . . . . . . . . 1

1.2 Power System Operation and Planning Overview . . . . . . . . . . . 2

1.3 The Challenge of Accommodating High Levels of Renewables . . . . . . . . 7

1.4 The Emerging Concept of Power System Flexibility . . . . . . . . . . 8

1.5 Flexibility Resources . . . . . . . . . . . . . . . . . . . . . 11

1.6 State of the Art on Flexibility Planning . . . . . . . . . . . . . . 12

1.7 Gaps in the State of the Art . . . . . . . . . . . . . . . . 15

1.8 Proposing the Flexibility Envelopes Concept . . . . . . . . . . . . . 17

1.9 Dissertation Outline . . . . . . . . . . . . . . . . . . . . . . 18

1.10 Claim Of Originality . . . . . . . . . . . . . . . . . . . . . 19

2 Traditional Planning with Flexibility Envelopes 22

2.1 Quantifying Operational Flexibility Requirements . . . . . . . . . . . . 23

2.1.1 The Periodogram of Flexibility Requirements . . . . . . . . . 23

2.1.2 The Flexibility Requirement Envelope _. . . . . . . . . . . 24

2.2 Quantifying Flexibility of Power System Resources . . . . . . . . . . . 27

2.2.1 Modeling Flexibility Potential Dynamics . . . . . . . . . . 27

2.2.2 The Flexibility Envelope of Power System Resources _ . . . . . . 28

2.3 Example: Traditional Planning with Flexibility Envelopes _ . . . . . . . . 29

2.3.1 Mathematical Program . . . . . . . . . . . . . . . . . . 30

2.3 .2 Problem Setup . . . . . . . . . . . . . . . . . . . . 34

2.3 .3 Results and Discussion . . . . . . . . . . . . . . . . 37

2.4 Comparison to Stochastic Planning . . . . . . . . . . . . . . . . . 38 
2.5 Summary . . . . . . . . . . . . . . . . . . . . . . 40

3 Energy-Centric Planning with Flexibility Envelopes 42

3.1 Introduction . . . . . . . . . . . . . . . . . . . . . 42

3.2 Quantifying Energy Flexibility Requirements . . . . . . . . . . . . . . 44

3.2.1 The Flexible Power Requirement Envelope . . . . . . . . . . . . . 45

3.2.2 The Flexible Energy Requirement Envelope - First Approach . . . . 48

3.2.3 The Flexible Energy Requirement Envelope-Second Approach _. 49

3.2.4 Comparison of the Two Approaches . . . . . . . . . . . . . 50

3.3 The Flexible Energy Envelope of Power System Resources _. . . . . . . . 51

3.4 Energy-Centric Planning with Envelopes . . . . . . . . . . . . . . 55

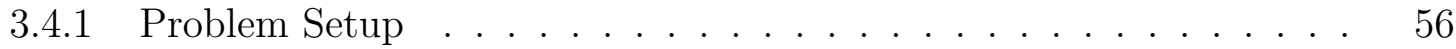

3.4 .2 Mathematical Program . . . . . . . . . . . . . . . . . 60

3.4 .3 Results and Discussion . . . . . . . . . . . . . . . . 64

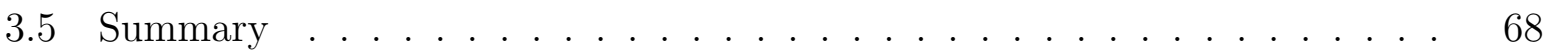

4 Probabilistic Planning with Flexibility Envelopes $\quad 70$

4.1 Introduction . . . . . . . . . . . . . . . . . . . 70

4.2 The Flexibility Requirement Envelope _. . . . . . . . . . . . . . . 73

4.3 The Flexibility Envelope of Power System Resources _ . . . . . . . . 75

4.4 Probabilistic Planning with Envelopes _ . . . . . . . . . . . . 77

4.4 .1 Problem Setup . . . . . . . . . . . . . . . 77

$4.4 .2 \quad$ Mathematical Program . . . . . . . . . . . . . . . . . 79

4.4 .3 Results and Discussion . . . . . . . . . . . . . . . . 83

4.5 Conclusion . . . . . . . . . . . . . . . . . . . . . . . . 88

5 Dynamical Planning With Flexibility Envelopes $\quad 90$

5.1 Introduction . . . . . . . . . . . . . . . . . . . . 90

5.2 Preliminaries . . . . . . . . . . . . . . . . . . . . . 92

5.2 .1 Variability vs Uncertainty . . . . . . . . . . . . . . . . . 92

5.2 .2 Relevance to Tracking Theory . . . . . . . . . . . . . . . . 93

5.2 .3 State-Space Interpretation . . . . . . . . . . . . . . . . 95

5.3 The Flexibility Envelope of a Power System Resource . . . . . . . . . . . 95

5.4 The Envelope of Flexibility Requirements . . . . . . . . . . . . . . . . 100 
5.5 Flexibility Adequacy Assessment _. . . . . . . . . . . . . . . . . . 105

5.5.1 Flexibility Aggregation . . . . . . . . . . . . . . . . . . 105

5.5 .2 Envelope Enclosure . . . . . . . . . . . . . . . . . . . . . . 106

5.5.3 Flexibility Adequacy of Envelope Enclosure . . . . . . . . . . 107

5.6 Dynamical Planning with Envelopes . . . . . . . . . . . . . . . . . . . 108

5.6.1 Min-Max Approach to Envelope Tracking . . . . . . . . . . . 108

5.6.2 Optimal Envelope Tracking Problem . . . . . . . . . . . . . . . 109

5.7 Application Example . . . . . . . . . . . . . . . . . . . . . . . . 111

5.7 .1 Problem Setup . . . . . . . . . . . . . . . . . . . . . 111

5.7 .2 Unit Commitment Problem . . . . . . . . . . . . . . . . . . 114

5.7.3 Unit Commitment Results and Discussion . . . . . . . . . . . . 118

$5.7 .4 \quad$ Economic Dispatch Problem . . . . . . . . . . . . . . . . 120

5.7.5 Economic Dispatch Results and Discussion . . . . . . . . . . . 121

5.8 Summary . . . . . . . . . . . . . . . . . . . . . . 121

6 Conclusion 123

6.1 Dissertation Overview . . . . . . . . . . . . . . . . . . . . . 123

6.2 Recommendations for Future Work . . . . . . . . . . . . . . . . . . 125

$\begin{array}{ll}\text { References } & 127\end{array}$ 


\section{List of Figures}

1.1 Timescales for power system operation and planning [1] . . . . . . . . . 2

1.2 Unexpected ramping event in the ERCOT power system jeopardizing the system's security $[2] . \ldots \ldots \ldots$. . . . . . . . . . . . . . 9

2.1 Schematic representation of the periodogram of wind generation output variability on a $\log -\log$ scale [3]. Note: $10^{-5} \mathrm{~Hz} \approx 24$ hours and $10^{-4} \mathrm{~Hz} \approx 1$ hour. 23

2.2 Schematic illustration of RES output consistency with its envelope . . . . 25

2.3 The flexibility requirement envelope arising from fitting the Laplace distribution to step changes of wind generation output as a function of $\tau$. . . 26

2.4 Schematic illustration of the concept of a flexibility dynamics for a scheduled flexibility resource. . . . . . . . . . . . . . . . . . . . 28

2.5 Illustrative example of a generic upward flexibility envelope. . . . . . . . . 30

2.6 Schematic representation of FE-RHED . . . . . . . . . . . . . . . . 31

2.7 Single realization of net load random process with caps on RES capacity and ramping rate. . . . . . . . . . . . . . . . . . . . 35

2.8 Histograms of EC and ENS for each dispatch approach. . . . . . . . . . . 39

3.1 Laplace PDF fitted to power step changes at $\tau=60$ minutes. . . . . . . . . 46

3.2 Standard deviation of power step changes as a function of $\tau$. . . . . . . . 47

3.3 Flexible power requirement envelopes plotted as integer multiples of the standard deviation $\sigma_{P} \ldots \ldots \ldots \ldots$. . . . . . . . . . . . . . . . . . 47

3.4 Laplace PDF fitted to energy step changes at $\tau=60$ minutes. . . . . . . . 48

3.5 Standard deviation of energy step changes as a function of $\tau$. . . . . . . 49

$3.6 \sigma_{E}$ (dashed) compared to $\sigma_{\Sigma P}(\tau)$ (solid) . . . . . . . . . . . . . 51

3.7 Sample power envelope of a thermal generation unit. . . . . . . . . . . . 52 
3.8 Sample power envelope of an energy storage unit. . . . . . . . . . . . 53

3.9 Energy envelope of the thermal generation unit as a function of $\tau$. . . . 54

3.10 Energy envelope of the energy storage unit as a function of $\tau$. . . . . 54

3.11 Schematic illustration of a single decision making epoch. . . . . . . . 56

3.12 Sample realizations of the net load random process bounded by the $90^{\text {th }}$ percentile flexible power envelope. . . . . . . . . . . . . 57

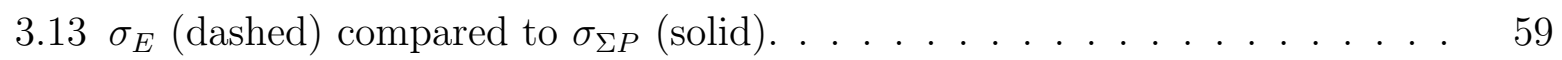

3.14 Integral of the sample scenarios in Fig. 3.12, enclosed by the $90^{\text {th }}$ percentile flexible energy requirement envelope. . . . . . . . . . . . . . 59

3.15 Histograms of generators' total dispatch cost under the three economic dispatch strategies. . . . . . . . . . . . . . 66

3.16 Histograms of the 25 MWh storage's state of charge. . . . . . . . . 67

3.17 Histogram of the 75 MWh storage's state of charge. . . . . . . . . . 69

4.1 Laplace PDF given $\mu=0$ and $b=1$. The dashed lines discretize the PDF uniformly by integer increments of $\sigma=\beta \sqrt{2} \ldots \ldots \ldots \ldots . \ldots 74$

4.2 The flexibility requirement envelopes of the BPA data for one hour duration, computed as integer increments of the standard deviation-Fig. $4.1 \ldots 76$

4.3 Schematic illustration of a single decision making epoch . . . . . . . . . 78

4.4 Fifty sample realizations of the net load random process (in blue) bounded by the flexibility requirement envelopes according to Table 4.1 (in red). . .

4.5 Stacked plot of the expected energy served (EES) by the $\mathcal{I}_{g}$ resources. The resources are stacked upward from the cheapest to the most expensive, starting from the bottom. . . . . . . . . . . . . . . . 85

4.6 Plot of expected energy not served (EENS) . . . . . . . . . . . 86

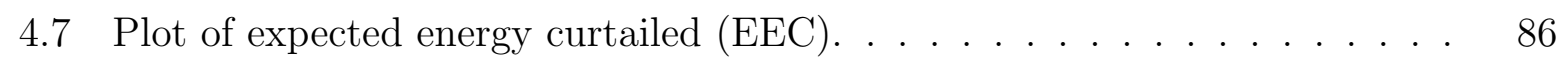

4.8 Plot of expected dispatch cost of the $\mathcal{I}_{g}$ resources. . . . . . . . . . . 87

4.9 Plot of overall expected cost including costs of EENS and EEC. . . . . . . 88

5.1 Schematic illustration of net load variability and uncertainty. . . . . . . . 93

5.2 Schematic illustration of the flexibility envelope of a resource. . . . . . . 96

5.3 Phase-plane characteristics of a power system resource, indicating the range of ramp rates the resource can sustain ( $y$-axis) depending on its output level

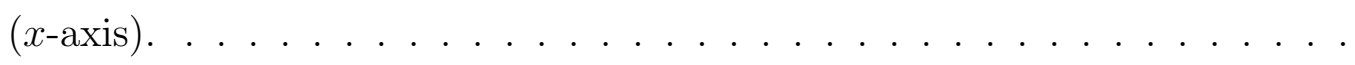


5.4 Flexibility envelope of a power system resource. . . . . . . . . . . . . . 100

5.5 Schematic illustration of the flexibility requirement envelope. . . . . . . . 101

5.6 Plot of $\left(\ell(k), \ell^{(1)}(k), \ell^{(2)}(k)\right)$ and their corresponding projections for 1000 randomly chosen samples from the BPA net load dataset. . . . . . . . . . . 102

5.7 Phase-plane characteristics of BPA's net load dataset. . . . . . . . . . . . . 104

5.8 Plot of the flexibility requirement envelope of BPA's net load dataset. . . . 105

5.9 Illustration of flexibility requirement envelope enclosure. . . . . . . . . . 106

5.10 Phase-plane characteristics of the net load. Solid lines represent the flexibility requirement envelope in the phase plane. . . . . . . . . . . . . . . 112

5.11 Time-domain plot of the flexibility requirement envelope and some sample net load random process realizations. . . . . . . . . . . . . . 113

5.12 Unit commitment solved repeatedly for a range of $\ell(0)$ values. Results for Portfolio 1 (solid). Results for Portfolio 2 (dashed). . . . . . . . . . . . . . 119 


\section{List of Tables}

2.1 Resources' Capacity, Ramping, and Cost Characteristics . . . . . . . . . 35

2.2 Expected Energy Served (EES) by Resources (MWh) . . . . . . . . . . . . 38

2.3 Expected Energy Not Served (EENS), Expected Energy Curtailed (EEC), Expected Net-Load Energy (ENLE) and Total Expected Cost Implications 38

3.1 Resources' Capacity, Ramping, and Cost Characteristics . . . . . . . . . 60

3.2 Storage Capacity, Power, and Efficiency Characteristics . . . . . . . . . . . 60

3.3 Expected Energy Dispatched and Expected Costs of Conventional Resources 65

4.1 Percentiles of the symmetric Laplace distribution for integer increments of the standard deviation . . . . . . . . . . . . . . . . . . . 75

4.2 Percentiles of the symmetric Laplace distribution for $20 \%$ increments of the percentiles ............................. 75

4.3 The standard deviation of the BPA data as a function of $\tau$. . . . . . . 76

4.4 Resources' Capacity, Ramping, and Cost Characteristics . . . . . . . . . . 80

4.5 Simulated Economic Dispatch Policies . . . . . . . . . . . . . . . . . 83

5.1 Characteristics of $n^{\text {th }}$-Order Differences (Values Are $\times 10^{2}$ ) $\ldots \ldots$. . . . 102

5.2 Operational Characteristics and Costs of Resources-Portfolio 1 . . . . . 112

5.3 Operational Characteristics and Costs of Resources-Portfolio 2 . . . . 113

5.4 Expected Energy Not Served (EENS), Expected Energy Curtailed (EEC), and Total Expected Cost $(\mathrm{EC}) \ldots \ldots \ldots$. . . . . . . . . 121 


\title{
List of Acronyms
}

\author{
MILP Mixed Integer Linear Program \\ RES Renewable Energy Source(s) \\ BPA Bonneville Power Administration \\ ERCOT Electric Reliability Council of Texas \\ FE-RHED Flexibility Envelopes Receding Horizon Economic Dispatch \\ SR-ED Economic Dispatch with Spinning Reserve Requirement \\ M-ED Myopic Economic Dispatch \\ ES Energy Served \\ EES Expected Energy Served \\ ENS Energy Not Served \\ EENS Expected Energy Not Served \\ EC Energy Curtailed \\ EEC Expected Energy Curtailed \\ ENL Energy of Net Load \\ EENL Expected Energy of Net Load \\ PDF Probability Density Function \\ PCA Principal Component Analysis
}




\section{Nomenclature}

\section{Indices}

$\tau$ Intra-hourly receding-horizon time.

$i$ Flexibility resources.

$h$ Unit commitment hour.

$k$ Economic dispatch period.

$m$ Discretization of $\tau$ such that $\tau=m T$.

$n$ Order of differentiation.

$q$ Discretization of the probability density function.

\section{Sets}

$\mathcal{V}$ Set of all plausible realizations of the net load.

$\mathcal{X}$ Set of all feasible trajectories of a resource.

$\mathcal{H}$ Set of all feasible trajectories of aggregated resources.

$\mathcal{I}_{g}$ Set of thermal generation resources.

$\mathcal{Q}$ Set of $p_{q}^{\text {th }}$ percentiles. 


\section{Continuous Functions of Time (Chapter 4)}

$v(\tau ; k)$ Single net load realization over $\tau$ as seen at time $k$.

$e_{v}^{\uparrow}(\tau ; k)$ Upward segment of the flexibility requirement envelope over $\tau$ as seen at time $k$.

$e_{v}^{\downarrow}(\tau ; k)$ Downward segment of the flexibility requirement envelope over $\tau$ as seen at time $k$.

$x(\tau ; k)$ Single resource trajectory over $\tau$ as seen at time $k$.

$e_{x}^{\uparrow}(\tau ; k)$ Upward segment of resource's flexibility envelope over $\tau$ as seen at time $k$.

$e_{x}^{\downarrow}(\tau ; k)$ Downward segment of resource's flexibility envelope over $\tau$ as seen at time $k$.

$h(\tau ; k)$ Single aggregate trajectory of resources over $\tau$ as seen at time $k$.

$e_{h}^{\uparrow}(\tau ; k)$ Upward segment of aggregate flexibility envelope over $\tau$ as seen at time $k$.

$e_{h}^{\downarrow}(\tau ; k)$ Downward segment of aggregate flexibility envelope over $\tau$ as seen at time $k$.

\section{Parameters}

$a_{i}$ No-load cost of resource $i$.

$b_{i}$ Incremental cost of resource $i$.

$R_{i}$ Ramp rate of resource $i$.

$\mu_{i}^{c}$ Charging efficiency of storage $i$.

$\mu_{i}^{d}$ Discharging efficiency of storage $i$.

$O R_{\tau}$ Operating reserve requirement of duration $\tau$.

$P_{i}^{\max }$ Maximum power output of resource $i$.

$P_{i}^{\min }$ Minimum power output of resource $i$.

$E_{i}^{\max }$ Maximum energy capacity of resource $i$.

$E_{i}^{\text {min }}$ Minimum energy capacity of resource $i$. 
$\lambda$ Net load envelope growth rate.

$\beta$ Laplace distribution scale factor.

$\gamma$ Cost discount rate.

$p_{q}$ The $p_{q}^{t h}$ percentile.

$W_{i}^{\uparrow}$ Upward segment of flexible energy requirement envelope.

$W_{i}^{\downarrow}$ Downward segment of flexible energy requirement envelope.

$H^{-}$Backward-in-time horizon length.

$H^{+}$Forward-in-time horizon length.

$T$ Sampling (discretization) period.

$\ell^{\text {min }}$ Minimum net load output.

$\ell^{\max }$ Maximum net load output.

$\ell$ Net load forecast.

$\ell^{(n)} n^{\text {th }}$-order forward difference of the net load time series.

$f_{v}, f_{x}$ Forcing functions of first-order ordinary differential equations (ODEs).

$\tau_{v}, \tau_{x, i}$ Time constants of first-order ODEs.

\section{Variables}

$u_{i}$ Commitment status (binary) of resource $i$.

$g_{i}, y_{i}$ Scheduled power output of resource $i$.

$E_{i}$ Energy state of resource $i$.

$r_{i}^{\uparrow}$ Upward flexibility potential of resource $i$.

$r_{i}^{\downarrow}$ Downward flexibility potential of resource $i$. 
$\hat{r}_{i}^{\uparrow}$ Upward flexibility envelope of resource $i$.

$\hat{r}_{i}^{\downarrow}$ Downward flexibility envelope of resource $i$.

$s_{i}^{\uparrow}$ Upward state of charge of storage $i$.

$s_{i}^{\downarrow}$ Downward state of charge of storage $i$.

$x_{i}^{\uparrow}$ Feasible upward deviation of resource $i$.

$x_{i}^{\downarrow}$ Feasible downward deviation of resource $i$.

$\boldsymbol{x}_{i}^{\uparrow}$ Upward state trajectory of resource $i$.

$\boldsymbol{x}_{i}^{\downarrow}$ Downward state trajectory of resource $i$.

$S^{\uparrow}, S^{\downarrow}$ Scheduling slack variables.

$e_{S}^{\uparrow}, e_{S}^{\downarrow}$ Envelope slack variables.

$\Delta P(\tau ; k)$ Step change in the power output of duration $\tau$ as seen at time $k$. $\Delta E(\tau ; k)$ Step change in the energy output of duration $\tau$ as seen at time $k$. 


\section{Chapter 1}

\section{Introduction}

\subsection{The North American Bulk Power System in Transition}

A worldwide trend is currently in effect, whereby power systems are moving away from fossil-based electricity production and towards renewable low carbon sources of electricity, primarily wind and solar power. Many countries have already set up ambitious targets for integrating renewable energy sources (RES). In the United States alone, over 145000 MW of additional generation capacity projected over the next decade is to be contributed by RES, thus amounting to a $700 \%$ increase in RES penetration levels, when compared to those of the year 2008 [4]. Such extreme RES integration efforts around the globe seek to harness the economic and environmental benefits of RES, mainly energy security and mitigating climate change [5].

The North American Electric Reliability Corporation (NERC) is primarily concerned with the reliable and efficient operation of the North American bulk power system, which prompted the organization to be on the frontier of addressing the challenges brought about by the need to accommodate high levels of RES. In one of their recent reports [4], NERC has concluded that "the North American bulk power system will require significant changes to traditional methods used for system planning and operation," to allow high penetration levels of RES in the near future without sacrificing system reliability. The primary driver behind such conclusion is the inevitable increase in variability and uncertainty in the power system, beyond what current practices were designed to handle. In response, this dissertation is an attempt at this challenge, by providing a mix of traditional and non-traditional paradigms catering to short-term operational planning under high RES penetration levels. 


\subsection{Power System Operation and Planning Overview}

Operation and planning in the North American bulk power system is hierarchical in nature, spanning several time frames, as illustrated in Fig. 1.1. The figure summarizes the time frames pertinent to the scope of this dissertation, which also happen to be the most impacted by the increased variability and uncertainty in the power system, as a result of RES integration.

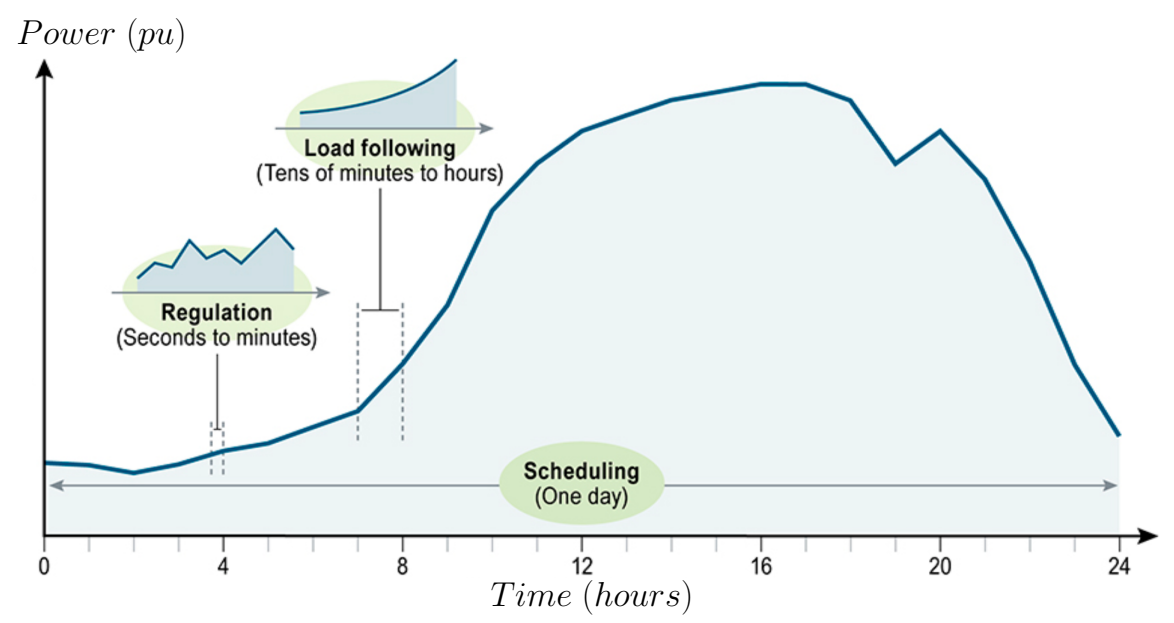

Fig. 1.1 Timescales for power system operation and planning [1].

\section{Long-term adequacy studies}

The North American bulk power system may as well be considered the biggest industrial system across North America. Such mega-structure would require several years of continuous planning ahead of time to accommodate generation growth and transmission expansion in line with demand growth. Traditionally, long-term adequacy studies have focused on generation capacity and transmission planning, while assuming sufficient ramping capability of resources. Subsequently, integration studies started to emerge, to evaluate the impact of RES penetration on power system operation in the long-term planning context [6]. Although integration studies ensured adequate capacity was available to cope with RES uncertainties, they fell short of evaluating the system's ability to use this capacity to meet variability over various time horizons [7]. Consequently, this prompted the recent emergence of power system flexibility studies to plan both capacity and ramping capability of 
resources. Flexibility studies are now considered the state of the art on long-term adequacy planning - surveyed later in this chapter. As conveyed by the short literature survey, this remains a wide open area of research, whereas classic capacity adequacy studies are yet to adapt to the multidimensional aspects of flexibility (i.e. capacity, ramping, and energy) in sustainable power systems. In this dissertation, we provide a flexibility assessment framework that can be integrated in production cost planning, to carry out long-term flexibility planning.

\section{Day-ahead scheduling (unit commitment)}

Unit commitment is concerned with determining the mix from a pool of resources, to be used in the upcoming day of operations. In North America, unit commitment is performed once a day, by which the generation schedules are due mid-day on the prior day. Resources are committed on an hourly basis and are scheduled such that their total power output meets the forecasted net load (i.e. load less non-dispatchable RES generation) for each hour of the day. Unit commitment also ensures that the committed resources have sufficient headroom/footroom to ramp up/down, to follow the intra-hourly deviations from the net load's hourly forecasts and to respond to system contingencies, i.e. sudden loss of generation and/or losses of transmission lines. This headroom/footroom capacity is termed operating reserve.

In the industry ${ }^{1}$, a typical mixed-integer linear program (MILP) representing this planning stage can be formulated as following:

$$
\min _{u_{i}, g_{i}} \sum_{h=1}^{24} \sum_{i \in \mathcal{I}_{g}} a_{i} u_{i}(h)+b_{i} g_{i}(h)
$$

\footnotetext{
${ }^{1}$ The power system industry in the United States relies primarily on mixed integer linear programming (MILP) to perform day-ahead power system planning.
} 
subject to

$$
\begin{array}{rlrl}
\sum_{i \in \mathcal{I}_{g}} g_{i}(h) & =\ell(h) ; & h=1, \ldots, 24 \\
\sum_{i \in \mathcal{I}_{g}} g_{i}(h)-x_{i}^{\downarrow}(h) & \geq \mathrm{OR}_{\tau}(h) ; & h=1, \ldots, 24 ; \tau=10 \\
\sum_{i \in \mathcal{I}_{g}} x_{i}^{\uparrow}(h)-g_{i}(h) \geq \mathrm{OR}_{\tau}(h) ; & h=1, \ldots, 24 ; \tau=10 \\
-60 \cdot R_{i} \leq g_{i}(h)-g_{i}(h-1) \leq 60 \cdot R_{i} ; & i \in \mathcal{I}_{g} ; h=2, \ldots, 24 \\
u_{i}(h) P_{i}^{\min } \leq x_{i}^{\downarrow}(h) \leq g_{i}(h) ; & i \in \mathcal{I}_{g} ; h=1, \ldots, 24 \\
g_{i}(h)-x_{i}^{\downarrow}(h) & \leq \tau \cdot R_{i} ; & i \in \mathcal{I}_{g} ; h=1, \ldots, 24 ; \tau=10 \\
g_{i}(h) & \leq x_{i}^{\uparrow}(h) \leq u_{i}(h) P_{i}^{\max } ; & i \in \mathcal{I}_{g} ; h=1, \ldots, 24 \\
x_{i}^{\uparrow}(h)-g_{i}(h) & \leq \tau \cdot R_{i} ; & i \in \mathcal{I}_{g} ; h=1, \ldots, 24 ; \tau=10
\end{array}
$$

where $g_{i}(h)$ is the scheduled output level of resource $i \in \mathcal{I}_{g}$ for the $h^{\text {th }}$ hour, $u_{i}(h)$ is the binary commitment state (on/off) of the $i^{t h}$ resource for the $h^{\text {th }}$ hour, $\left\{a_{i}, b_{i}\right\}$ are cost parameters, $\left\{P_{i}^{\max }, P_{i}^{\min }, R_{i}\right\}$ are the maximum output level (MW), the minimum input level (MW), and the maximum ramp rate (MW/minute) of the $i^{\text {th }}$ resource, $\ell(h)$ is the net load forecast for the $h^{\text {th }}$ hour, and $\mathrm{OR}_{\tau}(h)$ is the $\tau$-minutes operating reserve requirements for the $h^{\text {th }}$ hour.

The objective (1.1) is to minimize the commitment cost (via parameter $a_{i}$ ) and the energy generation cost (via parameter $b_{i}$ ) over the full day ahead (i.e. 24 hours), by committing the right mix of resources at each hour $h$. Constraint (1.2) ensures the power balance is met at each hour $h$, by matching the resources' dispatch to the hourly net load forecast. Constraints (1.3) and (1.4) ensure the operating reserve available from committed resources covers the $\tau$-minutes operating reserve requirements $\mathrm{OR}_{\tau}$ for the $h^{\text {th }}$ hour. Here, $\tau$ is usually taken to be 10 minutes, which represents combined primary and secondary frequency control reserve requirements. Constraint (1.5) ensures that the committed resources can follow the hourly step changes in the net load, subject to ramping constraints of resources, which represents the tertiary reserve requirement. Constraints (1.6)-(1.9) bound the $i^{\text {th }}$ resource's dynamics by its maximum ramping rate, maximum output level, and minimum output level. Finally, for the sake of simplicity, other constraints that are nontrivial (e.g. minimum uptime and downtime) or related to the transmission network 
are left out. Although such constraints are important in power system planning, they are not central to the conceptual development in this dissertation. A more complete MILP formulation of the traditional unit commitment problem can be found in [8].

Furthermore, the increased variability and uncertainty in the power system, as a result of accommodating high RES levels, has warranted the emergence of stochastic unit commitment methodologies [9]. These methodologies do not include constraints (1.3) and (1.4) in their formulation. Instead, the operating reserve is planned implicitly by imposing power balance on the probability-weighted stochastic scenarios. The advantage, here, over traditional planning with explicit reserve requirements is that stochastic unit commitment leads to a valuable reduction in planning costs in the face of increased variability and uncertainty [10]. In this dissertation, we propose another stochastic planning approach that circumvents the main drawback of current stochastic methodologies, namely the "curse of dimensionality."

\section{Load following (economic dispatch)}

Economic dispatch is concerned with determining power set points of resources, once they have been committed on the day before. The resources' output levels are updated on a sub-hourly basis, given the updated net load forecast and current commitment status of the power system resources. Typically, this process is carried out every 10 minutes, such that it provides sub-hourly tracking of the net load (a.k.a. load following), by deploying the planned operating reserve capacity. A traditional myopic linear program representing this planning stage can be formulated as the following:

$$
\min \sum_{i \in \mathcal{I}_{g}} b_{i} g_{i}(h+k)
$$

subject to

$$
\begin{aligned}
\sum_{i \in \mathcal{I}_{g}} g_{i}(h+k) & =\ell(h+k) & & \\
-10 \cdot R_{i} & \leq g_{i}(h+k)-g_{i}(h+k-1) \leq 10 \cdot R_{i} ; & & i \in \mathcal{I}_{g} \\
P_{i}^{\min } & \leq g_{i}(h+k) \leq P_{i}^{\max } ; & & i \in \mathcal{I}_{g}
\end{aligned}
$$


where $k$ is the economic dispatch period of the $h^{\text {th }}$ unit commitment hour. In this myopic formulation, the system operator needs only ensure that resources respect their ramp limits, when ramping from the previous economic dispatch period. At best, constraints (1.3) and (1.4), can be adapted to this formulation, to continuously replenish the operating reserve $\mathrm{OR}_{\tau}$ as time advances. The no-load cost $a_{i}$ and the unit commitment variable $u_{i}$ are removed from economic dispatch.

Moreover, there are further variations on the economic dispatch problem in the literature, such as predictive economic dispatch [11], and min-max economic dispatch [12]. Such predictive (non-myopic) strategies are in a better position to handle the increased variability and uncertainty as a result of RES integration. In this dissertation, we propose another predictive approach that captures and plans the intra-hourly reserve requirements, while performing economic dispatch in a receding horizon fashion.

\section{Operating reserve}

The net load is bound to deviate continuously from its forecast level in real time. This leads to power imbalances between supply and demand. For this reason, resources need to deviate from their scheduled output levels to follow the net load and maintain balance, via reserve deployment. Reserve may come from the committed resources (on-line or spinning), or it may come from off-line resources (non-spinning) that can be brought on-line on short notice. In North America, there are four main reserve types or categories, whereas, in reality, there is a lack of consensus across industry and academia on the exact categorization and operation of reserve types [13]. Without loss of generality, the four main types of operating reserve are primary (instantaneous), secondary (fast), tertiary (slow), and contingency reserves. Primary reserve is deployed within 30 seconds, via frequency-driven control, to follow the second-to-second variations in the net load with the objective of limiting frequency drift. Secondary reserve is deployed within 10 minutes, via economic dispatch, to follow the net load realizations (i.e. minute-to-minute variations). Tertiary reserve is deployed within an hour, often via supplementary unit commitment, to offset hourly net load realizations (i.e. hour-to-hour variations). Finally, contingency reserve is used in the event of sudden loss of generation or transmission outage. In North America, this reserve capacity is set to cover the loss of the largest generator - termed $N-1$ security.

With respect to constraints (1.3) and (1.4), there are several ways to calculating the 
operating reserve requirements $\mathrm{OR}_{\tau}$, which will be surveyed later in this chapter. The simplest method is to set $\mathrm{OR}_{\tau}$ to a certain percentile of the empirical distribution of the $\tau$-minute net load step changes (i.e. the net load forecast error). In this dissertation, we propose extending $\mathrm{OR}_{\tau}$ from being calculated for a single $\tau$ (e.g. only for 10 minutes duration) to being calculated over a spectrum of $\tau$ values covering the full unit commitment hour (i.e. $\tau$ ranging from 0 to 60 minutes). Conceptually, we argue in favor of defining the operating reserve as a spectrum, rather than categorically in terms of primary, secondary, and tertiary reserve types. That is, a spectrum of reserve is capable of accurately capturing the intra-hourly capacity and ramp requirements arising from variability and uncertainty in the net load, as we will see in the following chapter.

\subsection{The Challenge of Accommodating High Levels of Renewables}

Prevailing weather conditions dictate the output of most RES, thus making them variable and uncertain due to the erratic nature of weather patterns [7]. This poses a great challenge to system operators when having to balance power production and consumption, which must be carried out in real time. In fact, it is expected that current power system operation and planning practices will become grossly obsolete with the increasing penetration of RES in power systems around the globe $[14,3,15]$.

Traditionally, system operators have had to deal with variability and uncertainty resulting mainly from demand and random generation/transmission outages. Generally, they have always had enough lead-time and ample capacity to prepare generation in advance to deal with such circumstances [16]. On the contrary, under high RES penetration, the net load exhibits higher magnitudes and rates of variability [17] with higher uncertainty. This reduces the available lead-time, creates requirements for large and sudden ramping events, and causes frequent start-ups and shut-downs for dispatchable generating units [18, 19], thus warranting the rethinking of traditional operation and planning practices to suit better 1) shorter decision-making lead-times, 2) faster changeability, and 3) higher uncertainty. In addition, other resources in the system will be required to alter their generation and/or consumption in a variable manner and fast enough to overcome those three challenges - the basic premise behind power system flexibility.

Variability and uncertainty are two different but related issues. Variability arises because RES have maximum generation limits that change with time, while uncertainty arises 
because this limit cannot be known with perfect accuracy [20]. In particular, under high RES penetration, the impact of intra-hourly variability and uncertainty can become substantial enough to put significant stress on reserve requirements in terms of necessary capacity and its ability to ramp, both upward and downward. It is thus our ambition that all devices (producing and/or consuming power) in the power system can participate collectively in balancing power across the system, by exploiting their upward and downward flexibility-i.e. by providing flexible reserve.

One real world example, which highlights the potential risks associated with improper flexibility planning in a power system, is ERCOT's event on February 26, 2008 [2], when a large ramp-down of wind generation, an unexpected loss of conventional generation, and a quicker than expected load ramp-up occurred simultaneously, thus seriously threatening the security of ERCOT's system. The system operator had to call on reserve capacity and

load shedding by industrial customers, in exchange for economic compensation. The event lasted for 2 hours as shown in Fig. 1.2.

In this dissertation, we propose the concept of an envelope to capture the intra-hourly spectrum of reserve requirements arising from the net load variability and uncertaintytermed the flexibility requirement envelope. The height and slope of the envelope quantify capacity and ramp requirements, respectively. In the next chapter, we will require the aggregate flexibility envelope of all committed resources to enclose the flexibility requirement envelope, to ensure adequate system flexibility at any given time.

\subsection{The Emerging Concept of Power System Flexibility}

The concept of flexibility in power systems has been introduced formally only recently and has been recognized officially by organizations like the International Energy Agency (IEA) [5] and NERC [4, 21]. Concurrently, academic research and industry reports have begun to emerge and assess flexibility in power system operation and planning. Currently, the two main research frontiers in this area are in terms of long-term flexibility planning and shortterm operational flexibility scheduling. Currently yet, there is no universal definition of power system flexibility. Different authors and groups have contributed their own definition, which we discuss shortly here.

Flexibility is defined in [16] as "the ability of a system to deploy its resources to respond to changes in net load, where net load is defined as the remaining system load not served 


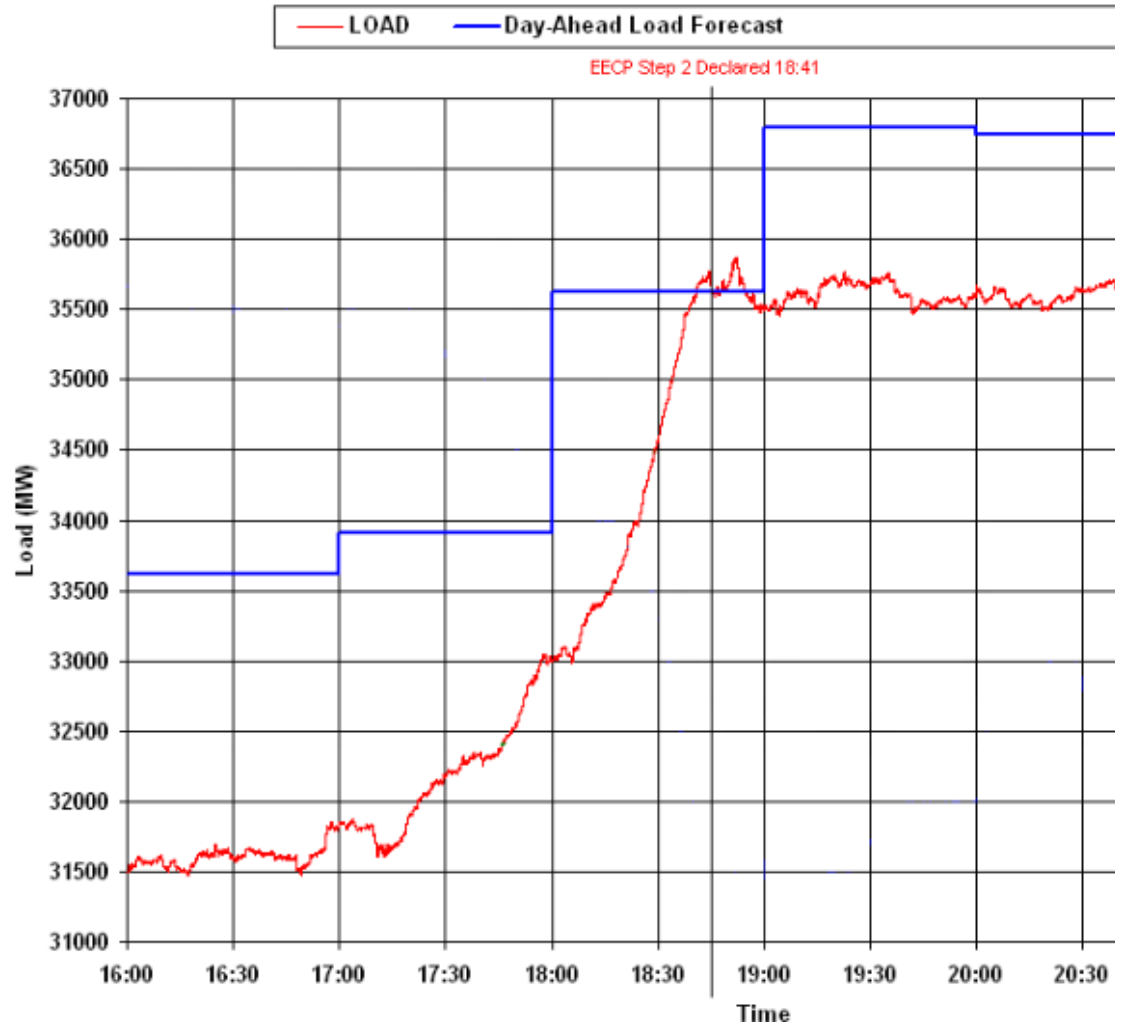

Fig. 1.2 Unexpected ramping event in the ERCOT power system jeopardizing the system's security [2]. 
by variable generation." A definition in [5] states that "flexibility expresses the extent to which a power system can modify its electricity production and consumption in response to variability, expected or otherwise." From an operational point of view, flexibility is seen in [3] as "the potential for capacity to be deployed within a certain time-frame" to respond to changes in net load. Similarly, [22] and [23] define operational flexibility "in terms of power capacity (MW), ramp rate $(\mathrm{MW} / \mathrm{min})$, that is, the ability to increase energy production with a certain rate, and ramp duration (min), that is, the ability to sustain ramping for a given duration." A lower level definition is adopted in [24], where flexibility is said to encompass controllability and observability information about the underlying power system which is constrained by the dynamics of its resources. Tying technical flexibility with economics, [19] defines flexibility as "the ability of a power system to cope with variability and uncertainty in both generation and demand, while maintaining a satisfactory level of reliability at a reasonable cost, over different time horizons." Similarly, flexibility is defined in [25] as "the system's capability to respond to a set of deviations that are identified by risk management criteria through deploying available control actions within predefined time-frame and cost thresholds."

Therefore, a power system must be flexible in its resources, operation, and planning to permit the integration of higher RES targets. The more flexible a power system is, the more variability and uncertainty it can handle, and the more RES levels can be achieved without sacrificing system reliability. This can be realized by diversifying the resources available in the power system, such that they exhibit various flexibility characteristics and flexibility costs, as argued in [26]. The challenge then becomes how to manage and optimize this flexibility potential according to system needs in the most economical way. The proposed concept of flexibility envelopes in this dissertation provides such means.

Having excess reserve capacity does not necessarily ensure power system security, especially under significant RES penetration [27]; this capacity must also be "flexible" enough to be deployed in good time. The system operator must validate the ramp-ability of reserve capacity implemented by its portfolio of resources, subject to transmission constraints. This portfolio has to be capable of providing power setpoint adjustments (both upward and downward), within the time scales and power volumes entailed by the realization of the net load variability and uncertainty, and within the transmission capacity of the network.

Moreover, the design of the transmission network can have a significant impact on the flexibility provided by resources. For instance, transmission switching technology has 
begun to emerge as a source of flexibility in the grid [28], while the locations of flexibility resources become highly relevant due to potential congestion [29]. In this dissertation, we do not perform transmission planning, nor do we consider the network's topology when performing flexibility planning. In the interest of time and scope, we are not addressing the effect of the transmission network on the proposed flexibility envelopes, nor the inherent flexibility of the grid itself. These are important matters which are planned for future research.

\subsection{Flexibility Resources}

Relying on existing resources in traditional power systems, which consists mainly of conventional generation units, is likely to be inadequate under high RES penetration [26]. Therefore, investors must seek to diversify their flexibility portfolio by including a wide range of technologies. Flexibility resources or technologies can be further classified into flexible generation, demand response, and energy storage.

Flexibility derived from flexible generation is mainly related to the capacity, ramp rate, and startup/shutdown time of the generating unit. Although a generator may be flexible in its capacity and ramping rate, this flexibility can be hindered by the reluctance of its owners to operate the resource in a variable and/or intermittent fashion, due to the wear and tear and increased fuel consumption. Manufacturers are constantly working on improving the cycling and ramping capabilities of this resource type. Others have investigated the cost effects of generation ramping in high wind penetration systems, in comparison to alternatives like demand response and energy storage systems [30].

Demand response refers to the ability of electricity consumers to alter their consumption patterns in response to net load variability. Demand response may occur involuntarily via imposed load shedding, or it may occur voluntarily via participation in demand response programs. Some types (e.g. industrial) can be called day ahead, while others (e.g. residential) can respond in real time [19]. Demand response is potentially a great flexibility resource due to the aggregate response of end users with a broad range of load devices of differing characteristics. Examples of demand side response are residential water heaters and electric vehicles.

Large-scale energy storage are increasingly becoming valuable flexibility resources as well. The ability to convert electricity to a stored form of energy has a unique advantage in 
the sense that electricity production does not have to be consumed anymore in real time, to maintain power balance. There are diverse types of energy storage technologies [31, 32] suitable for different roles in supporting the grid. For instance, batteries and flywheels can be used for regulation, while pumped hydro can be used for day-night arbitrage. Electric vehicles are also becoming a source of distributed energy storage [33], whereas there are some types of demand response devices (e.g. residential water heaters [34]) that may act as distributed energy storage, as well, due to their inherent storage capacity.

This dissertation proposes the concept of flexibility envelopes to capture and manage the flexibility (i.e. reserve flexibility) available from such resources. The height and slope of the envelope quantify capacity and ramping capability, respectively, of a single flexibility resource (or an aggregate). Adequate flexibility is ensured, when the aggregate flexibility envelop of resources encloses the flexibility requirement envelope arising from the net load variability and uncertainty, as we will see in the following chapter.

\subsection{State of the Art on Flexibility Planning}

\section{Short-term flexibility planning}

The objective of short-term flexibility planning is to ensure economical and feasible operations by committing the right power system resources and prepositioning them correctly ahead of time. This is generally done directly in the context of day-ahead unit commitment and predictive economic dispatch. With unit commitment, reserve capacity is scheduled and, in the case of economic dispatch, the available generation capacity is deployed to meet the current levels of net load. Inappropriate short-term operation planning may lead to insufficient flexibility in real-time operation - inadequate power capacity combined with potentially inadequate maneuverability - which in turn may lead to undesirable situations such as out-of-merit dispatch, involuntary load shedding, unnecessary renewable generation curtailment and, potentially, widespread blackouts. As mentioned above, unit commitment problem formulations generally attempt at resolving this problem. However, they only focus on getting the capacity right but not necessarily its ramping capability.

Conventionally, reserve types have been categorized according to their required deployment response time and the duration during which resources providing them have to maintain their deployed capacity. The three main categories are frequency containment 
reserves (primary/instantaneous), frequency restoration reserves (secondary/fast), and replacement reserves (tertiary/slow) [35, 36, 13, 37]. Such categorization is unlikely to guarantee system's security under significant RES penetration, because it does not accurately capture reserve requirements arising from the net load variability and uncertainty; a more systematic approach is desired.

Flexibility, on the other hand, quantifies in a more general way reserve capacity dynamics of power system resources over a time continuum - the horizon of potential flexibility deployment. This allows for a more rigorous consideration of reserve requirements in terms of both capacity and ramp-ability. The heuristic distinction between the various reserve types is no longer explicitly needed, thus allowing the various power system resources, including demand response and energy storage systems, to contribute collectively to the whole spectrum of reserve requirements. Henceforth, one must ask: to what extent does the recent literature on short-term planning quantify and plan reserve requirements in light of the emerging concept of flexibility?

Estimating a power system's flexibility requirements is directly related to quantifying and modeling the net load random process. This can be modeled exogenously or endogenously with respect to the planning problem. The exogenous approach quantifies the net load random process in terms of a probability distribution or a statistic (e.g. standard deviation), which is then used to impose an explicit reserve requirement constraint in the generation planning optimization model. This quantity could be static for all planning periods, or it could be dynamic and specific to each period. The endogenous approach models the net load random process stochastically (e.g. via a scenario-tree formulation), which is then integrated into the planning problem and solved via stochastic programming techniques. In the latter case, there is no explicit reserve requirement constraint, as it is accounted for implicitly via the need to satisfy the power balance constraint in each of the scenarios $[38,10]$.

In the case of an exogenous approach, some techniques map the probability distribution or its statistics directly to a reserve requirement for a certain timeframe such as the 10-minute spinning reserve $[14,39]$. Other techniques relax the reserve requirement by first mapping the probability to a reliability criterion [35, 40, 17, 41], which allows for insufficient flexibility as long as the reliability criterion is met. Yet other techniques further relax the reserve requirement by optimizing the reserve cost against the actual benefit of its availability [42, 27, 41]. The benefit is improved operational cost and/or feasibility 
of the planning problem, since directly mapping the net load random process to reserve requirements can be a stringent constraint during planning.

On the other hand, the endogenous approach for quantifying the net load random process is suitable for stochastic-based planning (e.g. stochastic unit commitment). The main advantage here is that fully hedging against scenarios that have much lower probability of occurrences may not be needed, considering the higher reserve costs associated with these scenarios [3]. The endogenous stochastic approach is surveyed thoroughly in [9].

\section{Long-term flexibility planning}

Interest in long-term flexibility assessment and planning has begun to emerge only recently to address the issue of flexibility adequacy. To this date, there are very few methodologies that have been proposed to assess the overall flexibility in power systems. They are surveyed in [43]. The main implication here is that flexibility consideration in long-term planning may change the optimal mix of resources to be invested in [44].

A high level flexibility assessment methodology was proposed in [5] tailored to inexperienced system operators, investors, and policy makers. The assessment tool did not require extensive data and computational resources. Assessment of flexibility and potential RES penetration targets were drawn from a number of qualitative and simplified quantitative analyses, given operational assumptions about systems in question. Results from one system could not be generalized to other systems, since each system was unique and the assessment results were system-specific.

On the other hand, production cost simulation can be used to gain more accurate and detailed assessment of flexibility. After simulating unit commitment and economic dispatch runs, statistical or probabilistic analysis can be applied to simulation results to assess the system's ability to match flexibility resources to flexibility requirements over various planning horizons. Operational and network constraints can be incorporated into the simulation runs, which provides an insight into their potential flexibility impacts.

The insufficient ramping resource expectation (IRRE) assessment method was proposed in [16] and took the production cost simulation approach. Periods of increasing, or separately decreasing, net load ramps were simulated to obtain net load and generation ramping time-series. The idea was to match ramping requirements over various time horizons to available flexibility from all resources and identify situations of potentially insufficient ramp- 
ability. The IRRE metric was proposed to replace the traditional loss of load expectation (LOLE) metric which assesses capacity inadequacy but not ramp inadequacy.

A more advanced technique was proposed in [19] which was also based on production cost simulation. The unit commitment and construction (UCC) algorithm determined the optimum portfolio of flexibility resources and the appropriate time to build and commission new resources. However, this came at a higher cost of computational complexity due to the added decision variables (to construct or not to construct).

In general, production cost simulation is data and computationally intensive due to having to incorporate unit commitment and economic dispatch runs. At best, data sampling must be taken somewhat widely apart in order to keep the computational tractability under control, thus reducing the accuracy of flexibility assessment. A much less computationally intensive alternative is to replace unit commitment runs with merit-order dispatch. The IRRE methodology was updated with merit-order dispatch in [45].

The key point to take from the above is that short-term flexibility greatly impacts longterm flexibility planning, especially when production cost simulation is deployed. This makes long-term planning studies prone to the limitations of current short-term planning methodologies, as elaborated next.

\subsection{Gaps in the State of the Art}

More or less, the various approaches above followed the conventional wisdom of quantifying reserve capacity for the 10-minute deployment reserves (i.e. primary and secondary reserve requirement) and for the one-hour deployment reserve (i.e. tertiary reserve requirement). At best, a constraint was imposed on those two categories to be able to linearly ramp to the full capacity within the entailed time frames. However, the essence of the concept of power system flexibility goes beyond merely defining reserve requirements statically for one or two time frames. Such approach does not satisfactorily capture the full intra-hourly characteristics (i.e. variability and uncertainty) of the net load random process, which may lead to underestimating flexibility requirements. In this dissertation, we attempt to fully capture these intra-hourly characteristics by proposing the flexibility requirement envelope concept capable of covering a spectrum of deployment time frames.

That being said, some recent approaches divert from the conventional wisdom. The "flying-brick" concept in [22] and the "probability-box" concept in [23] quantify reserve 
requirements based on statistical analysis of variability for a spectrum of intra-hourly durations. The proposed envelopes approach of this dissertation follows along the same lines but acknowledges the time evolution of reserve requirements, which resembles the shape of an envelope or a cone. Essentially, the "flying-brick" and the "probability box" are "boxed" versions of the proposed envelope, when transforming the envelope into the capacity-rampduration space. That is, the envelope carves out some of the empty space within the boxes. This empty space in the "flying" / "probability" boxes may end up imposing unnecessarily costly reserve requirements on the system.

Furthermore, the reviewed literature looks at operating reserve as deployable power (in megawatts). The proposed envelopes approach allows reassessment of operating reserve requirements from an energy-centric (as in megawatt-hours) perspective, which is lacking in the state of the art. Modeling the operating reserve and operating reserve requirements via envelopes allows us to switch between the traditional power-centric perspective and the proposed energy-centric perspective. One potential benefit of the energy-centric perspective is the ability to seamlessly integrate energy-constrained resources in planning, as elaborated in Chapter 3, as well as to facilitate planning for multi-carrier energy systems and energy hubs $[46,47,48,49]$.

Moreover, scenario-based stochastic planning techniques suffer from the "curse of dimensionality," which renders them intractable for realistic power system planning. On the other hand, adequate scenario construction and reduction can be difficult to achieve and add another layer of computational complexity to power system planning. In response to this challenge, in Chapter 4, we propose probabilistic envelopes-based operations planning. We propose the use of several probability-weighted envelopes that are easy to calculate from net load empirical data. We show how probabilistic envelopes planning circumvents the "curse of dimensionality," thus making it a viable and computationally tractable alternative to current stochastic planning approaches.

Last, the reviewed literature plans operating reserve in terms of average linear ramping requirements. The resources are modeled with maximum constant ramping, whereas the reserve ramp requirements are calculated statistically, on average, from empirical net load step changes. Chapter 5 proposes a complete restructuring of the current reserve paradigm, by casting short-term operational planning as a dynamical problem. The proposed envelopes framework is recast such that capacity and ramping are linked dynamically via differential algebraic equations. This leads to generalizing power system flexibility to 
higher-order dynamics, which allows for a more accurate representation of flexibility resources and requirements.

\subsection{Proposing the Flexibility Envelopes Concept}

The object of this dissertation is the presentation of a systematic framework and methodology to describe the behavior of power system resources' flexibility as needed by unit commitment and economic dispatch tools. Here, we define the flexibility of a power system resource as the power capacity this resource could potentially deploy in $\tau$ units of time later, as seen at time $t[3]$. It is better characterized by limit behavior as time progresses, which corresponds to a flexibility envelope or cone. Therefore, to ensure adequate system flexibility, the flexibility cone for the entire power system must enclose, reasonably well, the corresponding cone of flexibility needs arising from RES integration.

Via the flexibility envelope concept, we provide a new basis for the development of power system operation tools which could be used to (i) assess and visualize the probable range of RES excursions in terms of time (i.e. the flexibility requirement envelope), (ii) do the same for power system resources (i.e. the flexibility envelope) and to (iii) optimally match power system resources as countermeasures to RES variability and uncertainty. For instance, the work presented in [50], describing how the "flying-brick" concept integrates within an Energy Management System (EMS), could be redone in the same context using flexibility envelopes.

The developments, here, are carried out taking a North American power system operation planning approach, where an Independent System Operator (ISO) is in charge of operations planning. Nonetheless, the concept of flexibility envelopes could still be used in electricity systems where bilateral transactions and energy-only market-clearing are used to carry out short-term generation planning, as in most European countries. Envelopes could be useful primarily for transmission system operators and flexibility resource owners in preparation for the balancing mechanism. Flexibility requirement envelopes could be used by the TSO to determine how much flexibility resources need to be procured to run the balancing mechanism reliably and economically. Moreover, in the case of flexibility resource owners, they could calculate their own flexibility envelopes while trading off gate-closure market positions for wider or smaller ranges of flexibility to be offered in the balancing mechanism with the objective of profit maximization. Lastly, the concepts here 
are also compatible with the needs of vertically-integrated utilities who have a mandate to integrate significant amounts of non-dispatchable renewable generation.

\subsection{Dissertation Outline}

\section{Chapter 2: Traditional Planning with Flexibility Envelopes}

In this chapter, the flexibility envelopes framework is formulated. First, the flexibility requirement envelope is modeled, to capture the intra-hourly variability and uncertainty arising in the net load. Second, the flexibility envelope of a single resource is modeled, to capture the intra-hourly capacity and ramping capability of the resource. Third, planning with flexibility envelopes is formulated as a linear program (LP), in the context of recedinghorizon economic dispatch. The envelope-based economic dispatch is then compared to the traditional myopic economic dispatch and economic dispatch with short-term operating (spinning) reserve constraints. The comparison is carried out on an illustrative power system, using expected energy not served (EENS) and expected energy curtailed (EEC) metrics for benchmarking the performance of the three economic dispatch strategies. The formulation of the envelope-based planning is seen as an extension of the myopic economic dispatch LP formulation and can be seamlessly integrated with traditional planning tools.

\section{Chapter 3: Energy-Centric Planning with Flexibility Envelopes}

In this chapter, the flexibility envelope framework, presented in Chapter 2, is recast from an energy-centric perspective. First, the flexibility requirement envelope is remodeled, to capture the intra-hourly variability and uncertainty in the net load's energy random process. Second, the flexible energy envelope of a single resource is remodeled, to capture the intrahourly flexible energy available from the resource. Third, planning with energy envelopes is formulated as a mixed integer linear program (MILP), in the context of receding horizon economic dispatch. An illustrative power system, containing energy storage as a flexibility resource, is used to illustrate the advantage of energy-centric planning with envelopes. The re-formulation of the flexibility envelope framework remains linear and can also be seamlessly integrated with traditional planning tools. 


\section{Chapter 4: Probabilistic Planning with Flexibility Envelopes}

In this chapter, we extend the work of Chapter 2 to probabilistic planning, as an alternative to stochastic optimization. Whereas the approach in Chapter 2 planned deterministically against the worst-case scenario (i.e. the flexibility requirement envelope), by ignoring the probability distribution of the net load uncertainty, this chapter takes into account these probabilities by planning with respect to several flexibility requirement envelopes enclosing one another, each weighted by the percentile of scenarios it encloses. The advantage of such approach is circumventing the curse of dimensionality associated with stochastic optimization, as well as circumventing the complexity of constructing scenario trees and performing appropriate scenario reduction.

\section{Chapter 5: Dynamical Planning with Flexibility Envelopes}

In this chapter, the flexibility envelope framework, presented in Chapter 2, is completely reconstructed from a trajectory tracking perspective. First, the flexibility envelope of a single resource is modeled dynamically, to capture the higher-order dynamics of the resource. Second, the flexibility requirement envelope is modeled dynamically, as well, exhibiting higher-order derivatives, to capture the state-space characteristics of the net load's variability and uncertainty. Third, planning with dynamical flexibility envelopes is formulated as a min-max tracking problem to ensure higher-order flexibility adequacy. An example is formulated as a mixed integer linear program (MILP) in the context of unit commitment. The formulation of dynamical planning with envelopes remains linear, as well, allowing it to be seamlessly integrated with traditional planning tools.

\section{Chapter 6: Conclusion and Future Research}

In this chapter, concluding remarks are made on the flexibility envelopes framework and how it fits in the bigger picture of current and future power system planning. Furthermore, we discuss several research directions that extend the work presented in this dissertation.

\subsection{Claim Of Originality}

This dissertation makes several conceptual and methodological contributions to accommodating high RES levels in power system operation and planning applications. In regards 
to conceptualization, the dissertation presents a new paradigm for defining the operating reserve which is central to short-term operational planning:

1. It argues in favor of replacing traditional reserve types with a spectrum of reserve requirements, in line with the spectral analysis of the net load random process spanning multiple timescales. Traditional reserve types, mentioned earlier, permeate the state of the art on power system planning and do not necessarily capture the full intra-hourly characteristics of the net load's variability and uncertainty. In contrast, a spectrum of reserve requirements, in the form of an envelope, captures such characteristics precisely.

2. It redefines operating reserve in terms of energy-based reserve requirements, rather than the current power-based reserve requirements. This newly proposed energycentric perspective on operating reserve is suitable for today's emerging sustainable power systems that integrate energy-constrained flexibility resources, such as energy storage and demand response.

3. It generalizes to the concept of dynamical operating reserve exhibiting higher-order dynamics. This is a radical but necessary departure from the current operating reserve practices which define capacity and average linear ramping requirements. The newly proposed dynamical operating reserve paradigm is considered to be at the frontier of the aforementioned state of the art, as of the date of this dissertation.

On the other hand, the methodological contributions of this dissertation can be summarized as following:

1. Short-term planning with flexibility envelopes is formulated as linear and mixed integer linear programs. In these formulations, the dispatched/scheduled output levels of resources are prepositioned such that the aggregate flexibility envelope of resources encloses the flexibility requirement envelope, to ensure adequate system flexibility going forward.

2. In the case of the energy-centric approach, the dispatched/scheduled state of charge of energy-constrained devices are prepositioned, such that the aggregate flexible energy envelope of all resources encloses the flexible energy requirement envelope, to ensure adequate system flexibility moving forward. Here, the formulation remains a mixed integer linear program, as well. 
3. Probabilistic planning with flexibility envelopes is proposed as a viable alternative to pure scenario-based stochastic optimization for operations planning. Here, the aggregate flexibility envelope of resources must enclose, probabilistically, several flexibility requirement envelopes that cover different percentiles of the probability distribution of the net load uncertainty. The advantage of this approach is reducing operational costs arising from costly scenarios that have low probabilities of occurrence.

4. Dynamical planning with flexibility envelopes is cast as a min-max tracking problem. The dispatched/scheduled output levels of resources are prepositioned such that the can collectively track the maximum deviation in the net load random process, which happens to be its dynamical flexibility requirement envelope. The formulation remains a mixed integer linear program, as well. Dynamical short-term operational planning is considered a challenging problem that has never been formally tackled in the literature or in practice.

Moreover, we re-emphasize that the industry favors mixed integer linear programs to carry out power system planning. This dissertation adheres to this practice, when formulating power system planning with flexibility envelopes. 


\section{Chapter 2}

\section{Traditional Planning with Flexibility Envelopes}

Modern power systems are undergoing a transitional phase, increasingly incorporating renewable energy sources (RES) to harness their economic and environmental benefits. The main challenge with this transitional phase is the management of the increased variability and uncertainty in the power balance. Legacy operation and planning practices are gradually seen as becoming inadequate or ill-adapted in addressing this challenge. One particular gap in the state of the art, which is of a great importance, is estimating the operational flexibility potential of individual power system assets and their aggregation at the system level. System operators need to evaluate and plan ahead flexibility adequacy for their power systems in order to ensure feasible and economical operation under high RES penetration. Likewise, asset owners need to integrate the notion of asset flexibility as part of their investment and operations decisions. To this end, we propose the concept of the flexibility envelope to describe the flexibility potential dynamics of a power system and its individual resources in the operational planning time frame. We demonstrate that the resulting envelope dynamics can be a starting point for flexibility adequacy planning in systems with highly variable generation. 


\subsection{Quantifying Operational Flexibility Requirements}

\subsubsection{The Periodogram of Flexibility Requirements}

Statistically, several studies $[26,51,52]$ have shown that wind (and solar) generation output variations exhibit a considerable range of ramping characteristics as reflected by the frequency content of its power spectral density. Fig. 2.1 shows a schematic representation of the periodogram (a statistical estimate of the power spectral density) of wind generation output variations. There are three distinguishable parts in the periodogram [26, 3]. Part I essentially indicates that over long enough time spans (i.e. low frequencies), the wind generation output will vary on average between zero and full capacity (1.0 per unit). Part II exhibits a linear profile in log-log scale, which is known as the Kolmogorov spectrum [26]. The wind generation output variations roll off exponentially as $f^{-5 / 3}$, indicating that variations in the output decrease as timescales become shorter. Part III is flat at nearly zero magnitude, indicating that at high frequencies (i.e. very short time intervals), the output variations are essentially filtered out by the mechanical and electrical inertias of wind turbines.

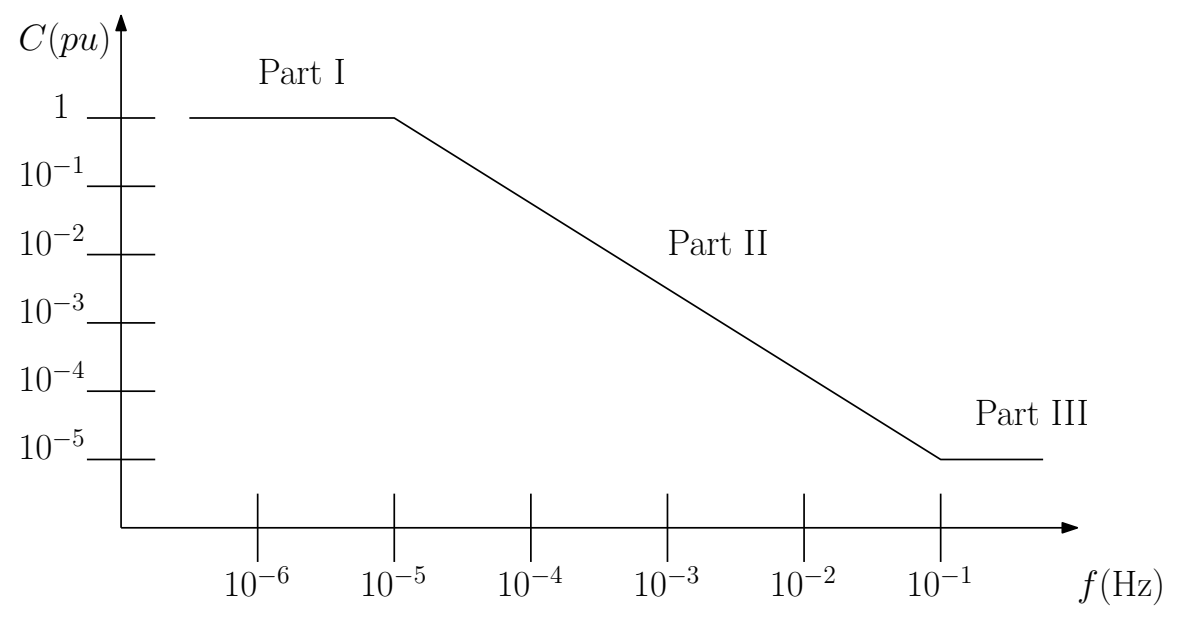

Fig. 2.1 Schematic representation of the periodogram of wind generation output variability on a log-log scale [3]. Note: $10^{-5} \mathrm{~Hz} \approx 24$ hours and $10^{-4} \mathrm{~Hz} \approx 1$ hour.

There are two main conclusions to take from this analysis: First, managing variability and uncertainty arising from RES integration requires a pool of resources with diversified flexibility characteristics to provide a matching spectrum of reserve dynamics [26, 3]. Sec- 
ond, it is not unlikely that the RES output can vary significantly within one hour. Given this empirical information, one can argue that there is inherently some value in exploiting the fact that RES output variations are well bounded within an hourly time frame.

Moreover, the state of the art literature, reviewed in Chapter 1, does not fully capture the variability and uncertainty characteristics of the net load for all timescales, as entailed by the frequency content of the periodogram. On the other hand, the proposed flexibility requirement envelope is capable of capturing such characteristics. Essentially, the flexibility requirement envelope is a transformation of the periodogram into the time domain, in the form of an envelope, whereby the envelope's height and slope bound the variability and uncertainty characteristics.

\subsubsection{The Flexibility Requirement Envelope}

The concept of an envelope implements the empirical findings illustrated above, by quantifying intra-hourly reserve capacity and ramp-ability requirements for a one hour period. Typically, the net load (i.e. load less RES output) forecast is held constant during each hourly planning period $h$, whereas the actual net load deviates from this forecast in real time ${ }^{1}$. These intra-hourly deviations during the $h^{\text {th }}$ hour, $\Delta P(\tau)$, are modeled here as step changes, with respect to the constant net load forecast, taken over a range of sub-hourly time durations $\tau \in[0,60]$ minutes:

$$
\Delta P(\tau ; h)=\ell(h+\tau)-\ell(h)
$$

where $\ell(h)$ represents the net load forecast for hour $h$ and $\ell(h+\tau)$ is the realization of the net load within hour $h$, measured $\tau$ minutes later.

A probability distribution of $\Delta P(\tau)$ can be computed empirically for every $\tau$, by constructing a relative frequency plot. This yields a stationary probability distribution that is a function of the variability calculation interval $\tau$ only, regardless of the hour $h$. Finally, plotting a certain percentile (e.g. the $95^{\text {th }}$ percentile) of the probability distribution for all $\tau$ yields an envelope that encompasses the vast majority of plausible realizations of reserve capacity requirements, whereas the slope of the envelope implicitly encompasses the

\footnotetext{
${ }^{1}$ Some systems consider the end of hour ramps to the next hour. Here, however, we assume an infinite ramp (slope) between the hourly net-load forecast step changes, while the actual inter-hourly ramp is handled by the flexibility requirement envelope.
} 
majority of plausible realizations of reserve ramping requirement.

It must be noted that we take a parametric approach in this dissertation to quantifying the flexibility requirement envelope. Other work, for example [23], argue in favor of the nonparametric approach, based on an empirical study of the poor goodness-of-fit performance of various parametric distributions. It is beyond the scope of this dissertation to assess the relative merits of each approach. The ultimate goal here is to have an adequate statistical representation of the behavior of the envelope.

Since $\Delta P(\tau)$ is assumed to be stationary random process (i.e. dependent on $\tau$ only), the per unit envelope is time-invariant and is, therefore, valid for all times and for all net load output levels. An implication of time-invariance is forward compatibility, as shown in Fig. 2.2, which means a sample realization cannot break the bounds of successive envelopes drawn at any time instant $\tau$. Therefore, enclosing the flexibility requirement envelope by the aggregate envelope of flexibility resources needs only be done at the beginning of the $h^{\text {th }}$ hour, to ensure adequate flexibility planning for the remainder of the same hour. However, in real-time operations (e.g. economic dispatch), the envelope must be optimized repeatedly, while the net load random process is being realized, because meritorder dispatch is in direct conflict with flexibility planning - it seeks to minimize dispatch costs, which may come at the expense of undermining future flexibility.

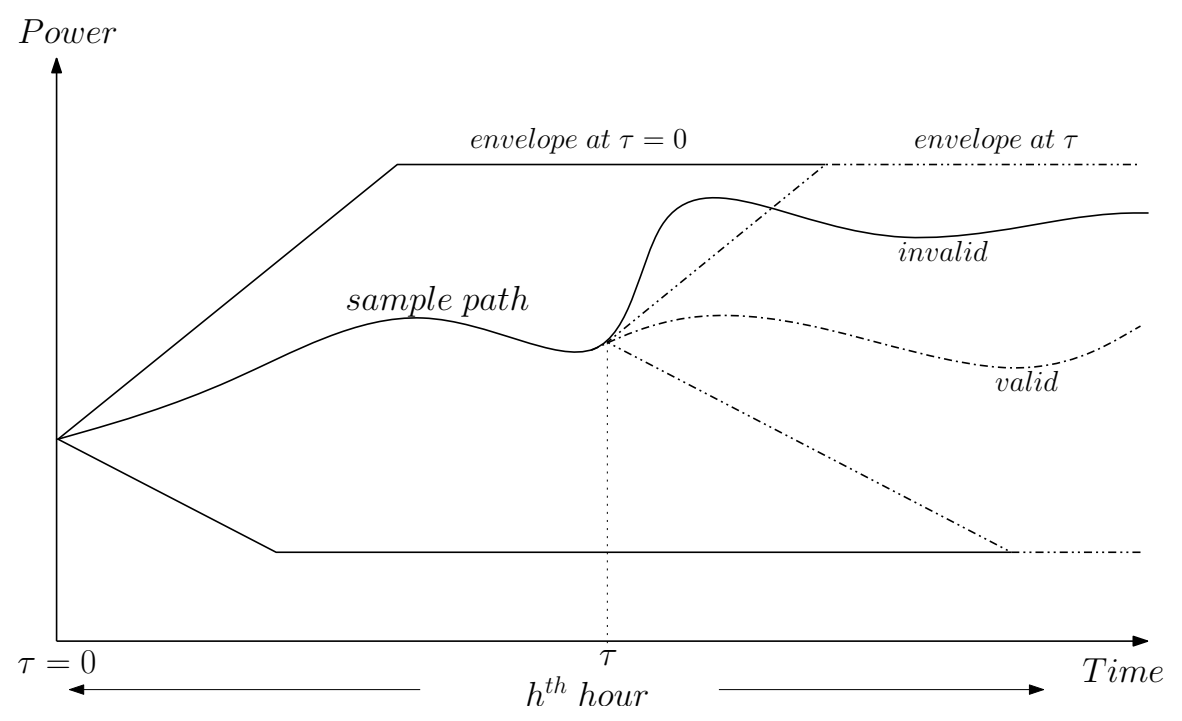

Fig. 2.2 Schematic illustration of RES output consistency with its envelope

On the other hand, it is possible for some renewable energy sources, such as solar power, 
the statistics of the output are dependent on time of day. In such case, the flexibility requirement envelope must be designed separately for each hour of the day, to avoid overly conservative flexibility planning when not needed; such as during night time when solar output variability and uncertainty are at minimum. This is a matter which lies outside of the scope of this dissertation, however.

\section{Example: Wind Power Variability}

It has been shown in $[51,53]$ that the wind generation variability random process is well fitted by a Laplace distribution. Fig. 2.3 plots the fitted Laplace distribution for the wind generation data from the Bonneville Power Authority (BPA) found in [51] for $\tau \in\{5,10,30,45,60\}$ minutes. The flexibility requirement envelope can be constructed by computing the interval, $\left[-\delta_{90 \%}^{-}(\tau), \delta_{90 \%}^{+}(\tau)\right]$, that encloses $90 \%$ of the probability using the Laplace probability density function. Fig. 2.3 shows the shape of the envelope for the wind generation data.

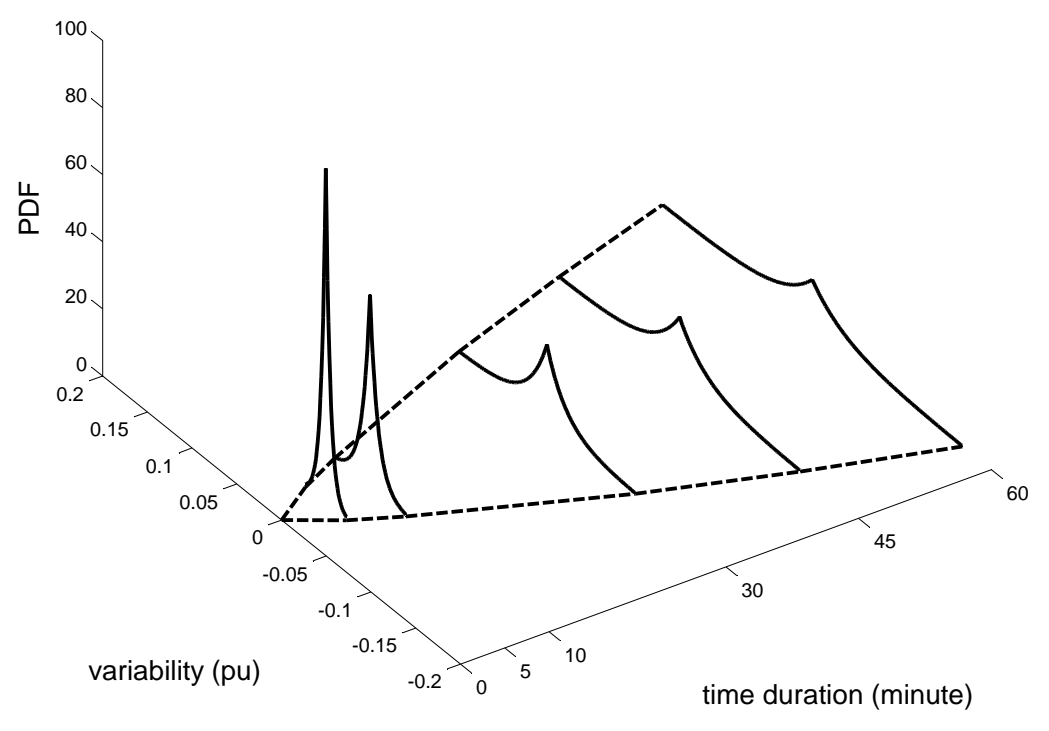

Fig. 2.3 The flexibility requirement envelope arising from fitting the Laplace distribution to step changes of wind generation output as a function of $\tau$

The choice of the percentage - $90 \%$ - enclosed within the envelope is arbitrary, here. In reality, the system operator must decide what percentage of probability to enclose within the envelope, based on further planning studies. On the other hand, the optimization mechanism presented later in this chapter allows for the envelope to be relaxed, to weigh 
the relative benefits of securing future flexibility needs at the expense of deploying much needed flexibility at the present time. Furthermore, Chapter 4 proposes a probabilistic envelopes planning approach that alleviates this issue.

\subsection{Quantifying Flexibility of Power System Resources}

We formulate next the dynamics of the flexibility envelope for a generic power system resource analogous to the flexibility requirement envelope. The flexibility envelope may also model an aggregation of resources such as a collection of smaller individual resources. The model abstracts from technology-specific details by describing the power output dynamics only in term of capacity and ramp-ability. However, complexities may arise primarily because the internal dynamics and constraints of the flexibility resource are nontrivial and time-dependent. Equally important, the flexibility envelope conveys the strong coupling of energy delivery and primary, secondary, and tertiary reserves.

\subsubsection{Modeling Flexibility Potential Dynamics}

To begin with, we distinguish between flexibility potential dynamics and flexibility envelope dynamics. Working in discrete-time where $\tau=m T$ for $m=0,1, \ldots, M$ such that $M T=60$ minutes, $r^{\uparrow}(m ; h)$ and $r^{\downarrow}(m ; h)$ describe, respectively, the upward and downward flexibility potential dynamics of a single resource evaluated at the $h^{\text {th }}$ hour for delivery $n$ intra-hourly time steps in the future. Both are quantified by the difference between the scheduled power trajectory $g(h)$ and a feasible upward $x^{\uparrow}(m ; h)$ or downward, $x^{\downarrow}(m ; h)$, deviation from the scheduled power trajectory - see Fig. 2.4. Therefore, the two coupled equations describing the flexibility potential dynamics of a single resource are:

$$
\begin{aligned}
& r^{\uparrow}(m ; h)=x^{\uparrow}(m ; h)-g(h) \\
& r^{\downarrow}(m ; h)=g(h)-x^{\downarrow}(m ; h)
\end{aligned}
$$

where $x^{\uparrow}(0 ; h)=x^{\downarrow}(0 ; h)=g(h)$ for all $h$.

Here, the scheduled output $g(h)$ is assumed to remain constant during each hour. A planned deviation from the constant level, to be distinguished from failures, is a realization of flexibility deployment via ED decisions and/or ancillary service deployment in real-time operation. More importantly, assuming that $g(h)$ stays constant during each hourly period 


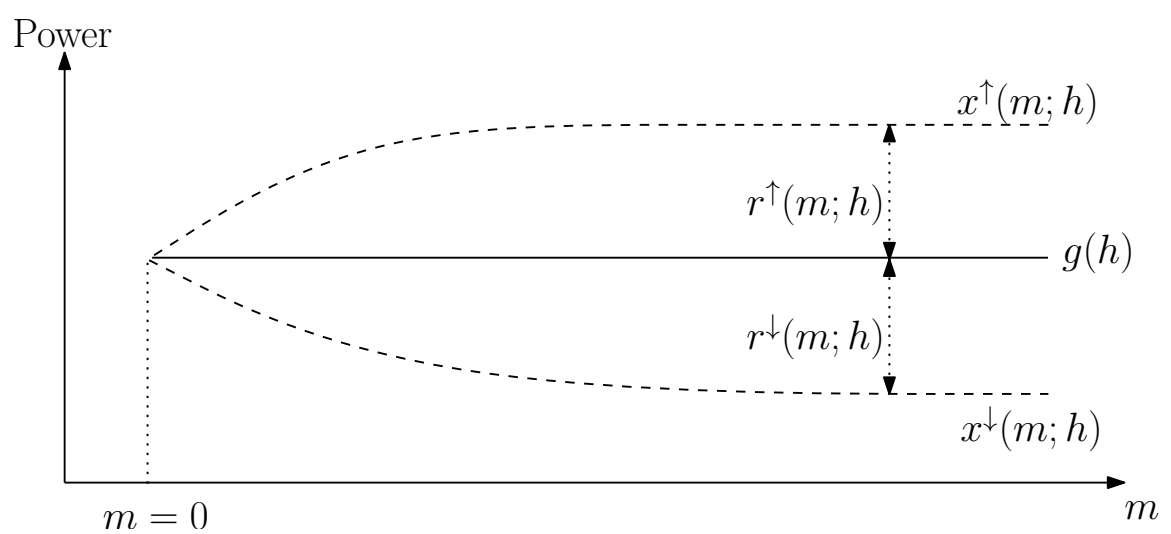

Fig. 2.4 Schematic illustration of the concept of a flexibility dynamics for a scheduled flexibility resource.

yields a time-invariant flexibility envelope, since the same maximum upward and downward deviations can be reproduced at any successive intra-hourly time step, $m$. This way, the flexibility model of resources is mathematically compatible with the flexibility requirement envelope model.

\subsubsection{The Flexibility Envelope of Power System Resources}

The flexibility envelope of a power system resource $\left(\hat{r}^{\uparrow}, \hat{r}^{\downarrow}\right)$ can be estimated recursively from the flexibility potential dynamics by maximizing the deviations $\left(x^{\uparrow}, x^{\downarrow}\right)$ at each subhourly time step $m$

$$
\begin{aligned}
& \hat{r}^{\uparrow}(m ; h)=\hat{r}^{\uparrow}(m-1 ; h)+\max \left\{x^{\uparrow}(m ; h): x^{\uparrow}(m ; h) \in \mathcal{X}^{\uparrow}(m ; h)\right\}-g(h) \\
& \hat{r}^{\downarrow}(m ; h)=g(h)-\hat{r}^{\uparrow}(m-1 ; h)-\max \left\{x^{\downarrow}(m ; h): x^{\downarrow}(m ; h) \in \mathcal{X}^{\downarrow}(m ; h)\right\}
\end{aligned}
$$

where the sets $\mathcal{X}^{\uparrow}(m ; h)$ and $\mathcal{X}^{\downarrow}(m ; h)$ represent the dynamical limitations of the power system resource in hour $h$ and intra-hour time step $m$.

For simplicity, if we consider linear time-invariant (LTI) power system resources only, we can identify four characteristics (constraints) describing the intra-hourly flexibility envelope dynamics, as illustrated in Fig. 2.5. These are $m$-step delays, (2.6) and (2.7), capacity, (2.8) and (2.9), ramping, (2.10) and (2.11), and energy storage constraints, (2.12) and (2.13), 
which apply for all $k$ and $m$

$$
\begin{gathered}
x^{\uparrow}(m ; h)=g(h) ; \quad 0 \leq m \leq d \\
x^{\downarrow}(m ; h)=g(h) ; \quad 0 \leq m \leq d \\
g(h) \leq x^{\uparrow}(m ; h) \leq P^{\max } \\
P^{\min } \leq x^{\downarrow}(m ; h) \leq g(h) \\
0 \leq x^{\uparrow}(m+1 ; h)-x^{\uparrow}(m ; h) \leq R^{\uparrow} \\
-R^{\downarrow} \leq x^{\downarrow}(m+1, h)-x^{\downarrow}(m ; h) \leq 0 \\
E(0 ; h)-T \sum_{j=0}^{m} x^{\uparrow}(j ; h) \geq E^{\min } \\
E(0 ; h)+T \sum_{j=0}^{m} x^{\downarrow}(j ; h) \leq E^{\max }
\end{gathered}
$$

where $d \geq 0$ is some response delay, $P^{\max }$ and $P^{\min }$ are respectively the maximum and minimum power levels of the resource, $R^{\uparrow}$ and $R^{\downarrow}$ are respectively its up and down ramp limits, $E^{\max }$ and $E^{\mathrm{min}}$ are the maximum and minimum energy storage levels of the resource while $E(0 ; h)$ is the initial energy stored in the resource at the beginning of hour $h$.

\subsection{Example: Traditional Planning with Flexibility Envelopes}

This simplified example illustrates the advantage of flexibility planning with economic dispatch in a rolling-horizon scheme. At every time step, the decision maker solves the conventional economic dispatch problem, while also ensuring that the projected aggregate flexibility envelope of committed resources encloses the projected flexibility requirement envelope, moving forward. The process is repeated at every economic dispatch time step, yielding a receding-horizon scheme. We shall call this the flexibility envelope recedinghorizon economic dispatch (FE-RHED). This approach will be compared to the myopic economic dispatch (M-ED) and the economic dispatch with a spinning reserve requirement 


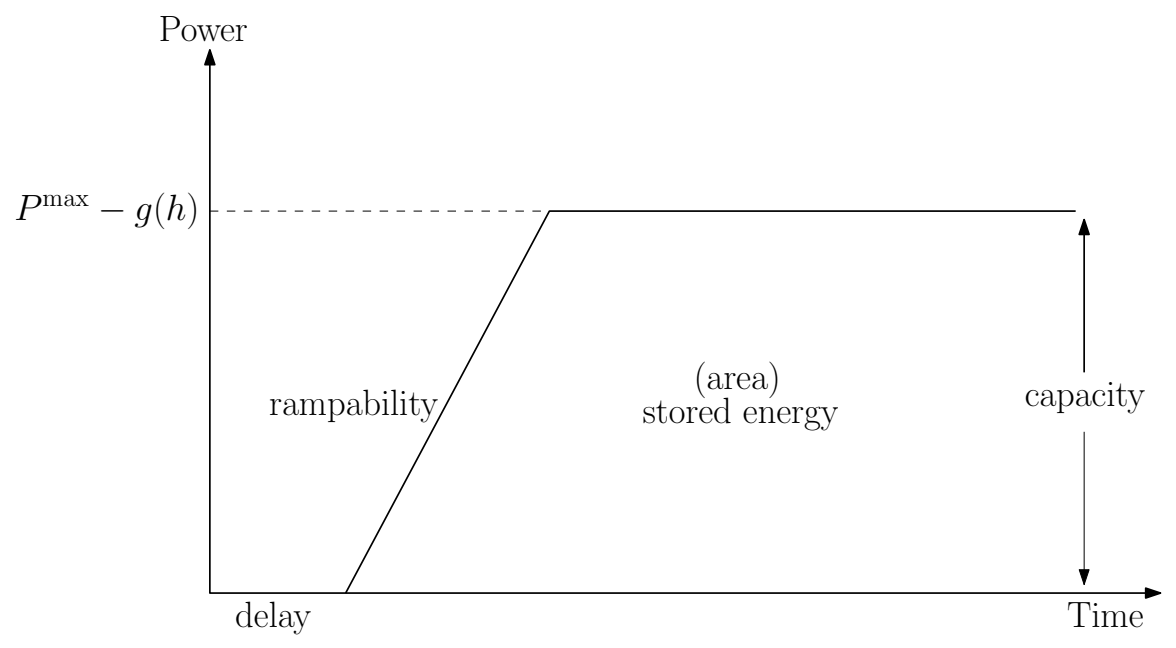

Fig. 2.5 Illustrative example of a generic upward flexibility envelope.

(SR-ED). The M-ED approach solves the conventional economic dispatch problem without considering spinning reserve or any flexibility requirement envelope. The SR-ED approach solves the conventional economic dispatch problem, while ensuring sufficient spinning reserve is available for the next 10 minutes.

Let there be $\mathcal{I}_{g}$ flexibility resources with generation levels $\left\{g_{i}\right\}_{i \in \mathcal{I}_{g}}$, maximum linear ramping rates $\left\{R_{i}\right\}_{i \in \mathcal{I}_{g}}$, and distinct incremental costs $\left\{b_{i}\right\}_{i \in \mathcal{I}_{g}}$. Let there be two slack resources capable of providing an infinite amount of flexibility, in both capacity and ramping. One slack, $S^{\uparrow}$, provides upward flexibility when there is generation deficiency. The other slack, $S^{\downarrow}$, provides downward flexibility when there is generation excess. The slack resources are priced at a much higher cost than the $\mathcal{I}_{g}$ resources and should be used as a last resort, when the economic dispatch problem is not feasible.

The long-term averages on the use of $S^{\uparrow}$ and $S^{\downarrow}$ are seen as estimates for the expected energy not served (EENS) and the expected energy curtailed (EEC), respectively. They will be used as metrics to assess the relative advantage of the FE-RHED approach against the SR-ED and M-ED approaches.

\subsubsection{Mathematical Program}

Fig. 2.6 illustrates the FE-RHED approach schematically. There are $K$ economic dispatch time steps within a single unit commitment hour $h$, of length $T=60 / K$ minutes (i.e. $T$ is the sampling period). For each dispatch, $\left\{g_{i}\right\}_{i \in \mathcal{I}_{g}}$, of the $\mathcal{I}_{g}$ flexibility resources, the 
individual and aggregate flexibility envelopes can be computed over a planning horizon of length $M$ future time steps, such that the planning horizon $\tau=m T$ and $m=1, \ldots, M$. The problem, hence, is to choose the cost-minimizing dispatch at the current time $h+k$ that will meet the current net load forecast, $\ell(h+k)$, while ensuring the aggregate flexibility envelope encloses the flexibility requirement envelope over the horizon $h+k+m$, where $m$ varies from 1 to $M$. Here, we assume that the envelope's horizon is discretized with the interval $T$ as well. This is repeated at every economic dispatch time step $h+k$, where $k$ varies from 0 to $K$. The following linear program describes this procedure for a single economic dispatch time step at $h+k$, given the previous optimal dispatch at $h+k-1$ and the projected envelope over the horizon $h+k+m$ for $m=1, \ldots, M$. For the sake of brevity next, $h+k$ is replaced with $k$, since $h$ is held constant as $k$ varies, while $h+k+m$ is replaced with $m$, since $k+h$ is held constant as $m$ varies. The following linear program describes this procedure for a single economic dispatch time step $k+n$, given the previous optimal dispatch at $k+n-1$ and the projected envelope over the horizon $k+n+m$ for $m=1, \ldots, M$ :

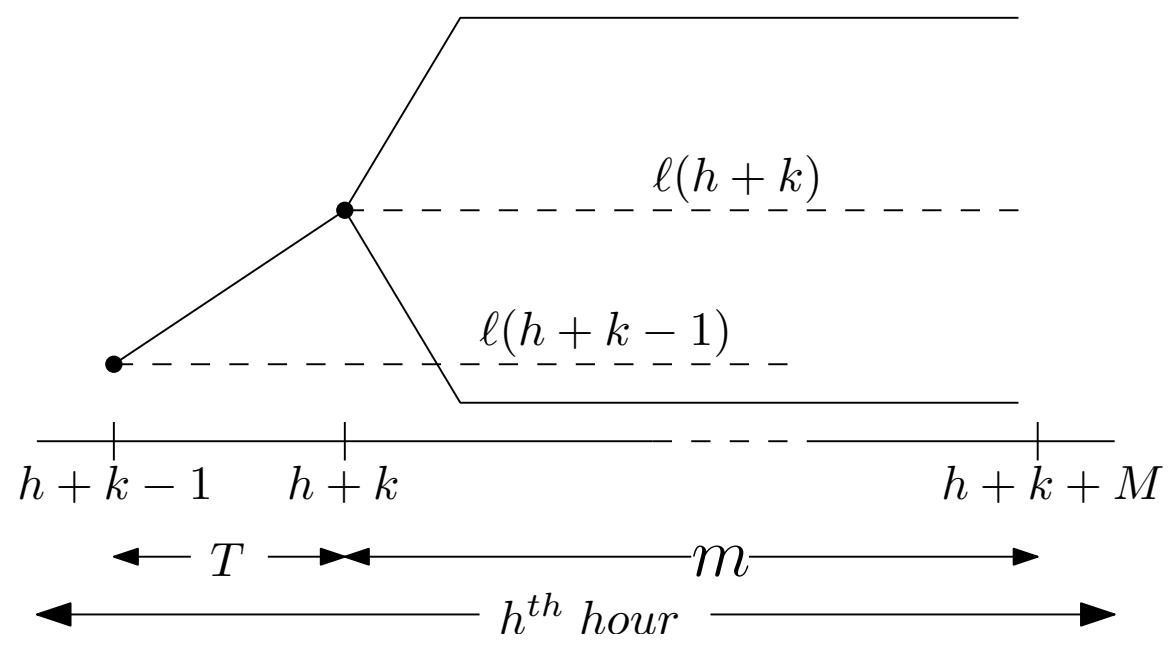

Fig. 2.6 Schematic representation of FE-RHED.

$$
\min \sum_{i \in \mathcal{I}_{g}} b_{i} g_{i}(k)+c_{S}\left[S^{\uparrow}(k)-S^{\downarrow}(k)\right]+\sum_{m=1}^{M} \gamma(m) c_{S}\left[e_{S}^{\uparrow}(m)-e_{S}^{\downarrow}(m)\right]
$$


this is subject to

Dispatch constraints at $k$

$$
\begin{array}{rlrl}
\sum_{i \in \mathcal{I}_{g}} g_{i}(k) & +S^{\uparrow}(k)+S^{\downarrow}(k)=\ell(k) & \\
g_{i}^{\min } & \leq g_{i}(k) \leq g_{i}^{\max } ; & & i \in \mathcal{I}_{g} \\
-R_{i} & \leq g_{i}(k)-g_{i}(k-1) \leq R_{i} ; & & i \in \mathcal{I}_{g} \\
S^{\uparrow}(k) & \geq 0 & & \\
S^{\downarrow}(k) & \leq 0 & &
\end{array}
$$

Resource Flexibility Envelopes for $m=1, \ldots, M$ and $i \in \mathcal{I}_{g}$

$$
\begin{gathered}
g_{i}^{\min } \leq x_{i}^{\downarrow}(m) \leq g_{i}(k) \\
g_{i}(k) \leq x_{i}^{\uparrow}(m) \leq g_{i}^{\max } \\
-m \cdot R_{i} \leq x_{i}^{\downarrow}(m)-g_{i}(k) \leq 0 \\
0 \leq x_{i}^{\uparrow}(m)-g_{i}(k) \leq m \cdot R_{i}
\end{gathered}
$$

Envelopes Matching for $m=1, \ldots, M$

$$
\begin{aligned}
\sum_{i \in \mathcal{I}_{g}}\left(x_{i}^{\uparrow}(m)-g_{i}(k)\right)+e_{S}^{\uparrow}(m) & \geq \min \left(\ell^{\max }-\ell(k), \sqrt{m} \lambda\right) \\
\sum_{i \in \mathcal{I}_{g}}\left(g_{i}(k)-x_{i}^{\downarrow}(m)\right)-e_{S}^{\downarrow}(m) & \geq \min \left(\ell(k)-\ell^{\min }, \sqrt{m} \lambda\right) \\
e_{S}^{\uparrow}(m) & \geq 0 \\
e_{S}^{\downarrow}(m) & \leq 0
\end{aligned}
$$

There are three distinct sets of constraints in the above problem. Constraints (2.15)(2.19) ensure the combined resources balance the net load at time step $h+k$, while respecting the capacity ramping limitation of resources as they ramp from their previous dispatch at $h+k-1$. Constraints (2.20)-(2.23) bound the flexibility envelope of each resource $i \in \mathcal{I}_{g}$ at time steps $h+k+m$, where $m$ varies from 1 to $M$, again while respecting the capacity and ramping limitations of the resources. Constraints (2.24)-(2.27) ensure the aggregate flexibility envelope encloses the flexibility requirement envelope at time-steps $h+k+m$. The 
evolution of the upper and lower half-envelopes of flexibility requirement over the receding horizon are described in the right-hand sides of (2.24) and (2.25), respectively. Here the half envelopes grow at the rate of $\lambda \mathrm{MW} / T$ and saturate once the maximum and minimum possible net load are reached.

We emphasize here that the problem (2.14)-(2.27) is solving the economic dispatch at time $h+k$ projecting the potential evolution of the net load up to $M$ sub-hourly time steps ahead. Therefore, envelopes are always calculated based on the current state of the system when the economic dispatch is computed. So, if in between the current dispatch time and the previous sub-hourly dispatch step flexibility has been used, the envelopes of resources which have deployed flexibility are updated based on their current operating point.

The primary objective (2.14) is to minimize the cost of dispatch associated with the current dispatch time step $h+k$, while enforcing the aforementioned three sets of constraints. Ideally, the $\mathcal{I}_{g}$ resources should have enough flexibility to ramp from the previous time step $h+k-1$, while ensuring there is still enough flexibility to enclose the projected flexibility requirement envelope, moving forward. However, due to poor planning in the past or a lack of sufficient flexibility, the $\mathcal{I}_{g}$ resources may not be able to collectively position themselves at time step $h+k$ to balance the current net load or to ensure proper enclosure of the projected flexibility requirement envelope. Consequently, the decision maker may have to deploy slack resources [in the form of load shedding $\left(S^{\uparrow}\right)$ and generation curtailment $\left(S^{\downarrow}\right)$ ] to avert dispatch infeasibility.

Deploying slack resources is undesirable and thus is priced much higher than the other resources. They are to be used as a last resort when the problem becomes infeasible. On the other hand, feasibility at $h+k$ can be improved by relaxing the flexibility requirement envelope; this is done via the slack variables, $e_{S}^{\uparrow}$ and $e_{S}^{\downarrow}$, in (2.24)-(2.27). Relaxing future flexibility requirement comes at a cost, since it may lead to poor prepositioning of the $\mathcal{I}_{g}$ resources at the current time, which, in turn, may affect future flexibility availability. Therefore, the cost of envelope relaxation is reflected in the third term of (2.14). The cost could also be discounted at a rate of $\gamma(m)$ to reflect that near-term flexibility is more valuable than long-term flexibility. A reliability criterion [17] or a cost/benefit analysis [54] can also be used to limit the use of the slack resources.

Thus, the problem in (2.14)-(2.27) becomes that of balancing the current flexibility requirement (i.e. the ability to ramp from the previous dispatch time step to a feasible positioning at the current time step) with future flexibility requirements (i.e. enclosing the 
projected flexibility requirement envelope), while balancing the system's current net load. The trade-off depends on how the $\mathcal{I}_{g}$ resources, the slack resources and the envelope's slack variables are priced relative to one another in (2.14).

Last, the mathematical program presented here precludes modeling of energy-constrained devices and assumes all resources are conventional thermal generation units. Chapter 3 focuses specifically on energy-constrained planning with envelopes.

\subsubsection{Problem Setup}

A toy system consisting of five flexibility resources will be used to simulate the dispatch operations of a single hour, with the objective of showing that the FE-RHED approach leads to less load shedding and less generation spillage than the M-ED and SR-ED approaches. Time Resolution: The hour is discretized into $K=12$ time steps of length $T=5$ minutes. This means dispatch decisions are obtained every 5 minutes, and the net load varies randomly every 5 minutes as well. The projected envelopes are discretized at 5 -minutes for $M=12$, meaning that at each economic dispatch step we are projecting one hour into the future.

Net Load Model: The forecast assumes the load is constant at $50 \mathrm{MW}$ throughout the hour. The RES output varies randomly according to a Laplace distribution characterized by $\beta=10 \mathrm{MW} / 5$ minutes. There is $40 \mathrm{MW}$ of RES capacity, and the RES maximum

5 -minute step change is $\lambda=1.63 \cdot \sqrt{2} \cdot 10=23.05 \mathrm{MW} / 5$ minutes. This corresponds to the $95^{\text {th }}$ percentile of the Laplace distribution. Therefore, the net load $\ell(h+k)$ can vary between $10 \mathrm{MW}$ and $50 \mathrm{MW}$, and the 5-minute step changes in the RES realizations are bounded between 0 and $\lambda$. The RES forecast for the hour is $20 \mathrm{MW}$, meaning that $\ell(h+k)=30 \mathrm{MW}$ for $k=0$ - see, for example, Fig. 2.7.

Flexibility Resources: There are five identical flexibility resources which are given fixed incremental costs. There are two slack resources priced at a much higher cost than the other five resources. Table 2.1 shows the capacity, ramping, and cost characteristics of flexibility resources, slack resources and envelopes' slack variables. The combined ramping of flexibility resource is two thirds of $\lambda$ computed above, to simulate flexibility scarcity.

Dispatch Policies: The linear program described in (2.14)-(2.27) is used to simulate the FE-RHED, M-ED, and SR-ED approaches for $K=12$ time steps. In the case of FE-RHED, the horizon is set to one hour, $M=12$. In the case of M-ED, the horizon length is set 

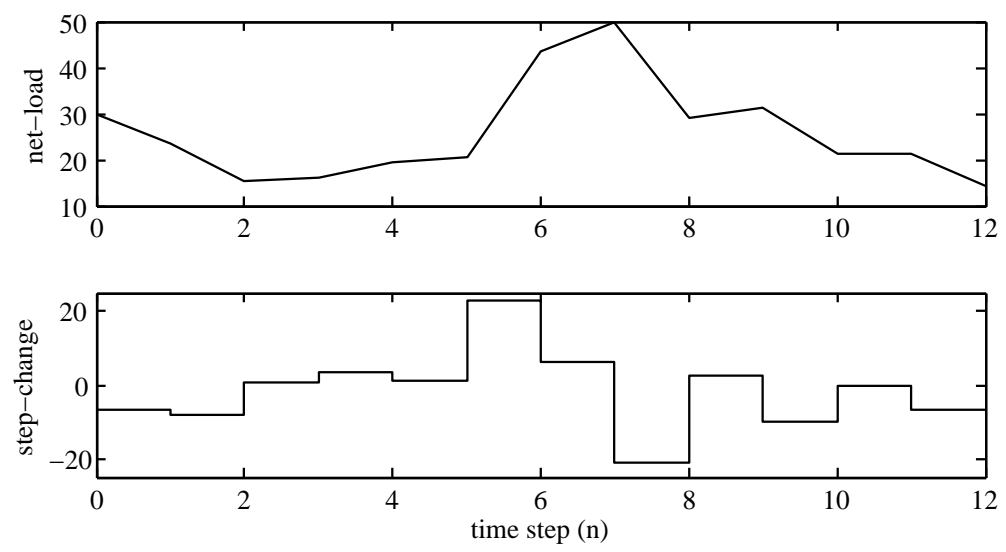

Fig. 2.7 Single realization of net load random process with caps on RES capacity and ramping rate.

Table 2.1 Resources' Capacity, Ramping, and Cost Characteristics

\begin{tabular}{ccccc}
\hline & $g^{\max }(\mathrm{MW})$ & $g^{\min }(\mathrm{MW})$ & $R(\mathrm{MW} / 5 \mathrm{~min})$ & $b(\$ / \mathrm{MWh})$ \\
\hline 1 & 10 & 2 & 3.07 & 20 \\
2 & 10 & 2 & 3.07 & 40 \\
3 & 10 & 2 & 3.07 & 60 \\
4 & 10 & 2 & 3.07 & 80 \\
5 & 10 & 2 & 3.07 & 100 \\
$S^{\uparrow}$ & $\infty$ & 0 & $\infty$ & 1000 \\
$S^{\downarrow}$ & 0 & $-\infty$ & $\infty$ & 1000 \\
$e_{S}^{\uparrow}$ & $\infty$ & 0 & $\infty$ & 1000 \\
$e_{S}^{\downarrow}$ & 0 & $-\infty$ & $\infty$ & 1000
\end{tabular}


to zero, such that there is no reserve planning looking forward. In the case of SR-ED, the horizon length is set to 10 minutes with a 10 minute discretization, such that there is only one step looking forward to plan the 10-minute reserve as per NERC's requirement. For all policies, the discount factor $\gamma(m)$ in (2.14) is set to one.

Performance Metrics: To assess the quality of the dispatch decisions made through the various policies, we calculate the energy served for each realization $j\left(\mathrm{ES}_{i, j}\right)$ and the expected energy served $\left(\mathrm{EES}_{i}\right)$ by each resource $i$. In addition, we compute the energy not served $\left(\mathrm{ENS}_{j}\right)$ and the energy curtailed $\left(\mathrm{EC}_{j}\right)$ for each realization $j$, from which we obtain the expected energy not served (EENS) and the expected energy curtailed (EEC) for all $J$ realizations of the net load random process.

$$
\begin{aligned}
\mathrm{ES}_{i, j} & =T \sum_{k=1}^{K} g_{i, j}(k) ; & i=1, \ldots, I ; j=1, \ldots, J \\
\mathrm{EES}_{i, J} & =\frac{1}{J} \sum_{j=1}^{J} \mathrm{ES}_{i, j} ; & \\
\mathrm{ENS}_{j} & =T \sum_{k=1}^{K} S_{j}^{\uparrow}(k) ; & \\
\mathrm{EENS}_{J} & =\frac{1}{J} \sum_{j=1}^{J} \mathrm{ENS}_{j} & \\
\mathrm{EC}_{j} & =T \sum_{k=1}^{K}-S_{j}^{\downarrow}(k) ; & \\
\mathrm{EEC}_{J} & =\frac{1}{J} \sum_{j=1}^{J} \mathrm{EC}_{j} &
\end{aligned}
$$

where $g_{i, j}(k), S_{i, j}^{\uparrow}(k)$ and $S_{i, j}^{\downarrow}(k)$ correspond to the realizations of the economic dispatch decisions at time step $k$ for random net load realizations $j=1, \ldots, J$.

Because of the power balance imposed by (2.15), the sum of the expected values (2.29)(2.33) should converge to the expected net load energy (ENLE) requirement

$$
\operatorname{ENLE}_{J}=\frac{T}{J} \sum_{j=1}^{J} \sum_{k=1}^{K} \ell_{j}(k)
$$


which should be $30 \mathrm{MWh}$ given the forecasts for the load (50 MW) and the variable generation $(20 \mathrm{MW})$. To assess convergence of the dispatch simulations, we calculate the incremental change in $\mathrm{ENLE}_{J}$ and ensure that it is less than the desired accuracy $(\epsilon=0.01$ MWh here)

$$
\left|\mathrm{ENLE}_{J}-\mathrm{ENLE}_{J-1}\right|<\epsilon .
$$

Hence, once we have simulated $J$ realizations of the net load random process and that it affects the value of $\mathrm{ENLE}_{J}$ by less than $\epsilon=0.01 \mathrm{MWh}$, we stop.

\subsubsection{Results and Discussion}

Table 2.2 displays the EES values for each of the flexibility resources for the hour of operation and for each economic dispatch approach. Table 2.3 shows the EENS, EEC, and ENLE values for each economic dispatch approach as well. The percentage changes are taken with respect to the M-ED approach. By inspection of the tables, we can see how imposing a spinning reserve requirement in SR-ED shifts part of the dispatch from cheaper resources to the more expensive ones. Imposing the envelope in FE-RHED further shifts the dispatch from cheaper resources to the more expensive ones. Out-of-merit dispatch operation leads to an increase in cost as shown in Table 2.2, but leads to a decrease in load shedding (EENS) and RES curtailment (EEC), as seen in Table 2.3. Although there has been an increase in the dispatch cost, we see that the overall system cost (accounting for dispatch, load shedding and generation curtailment) has dropped in a non-negligible fashion with both SR-ED and FE-RHED.

By inspection of Fig. 2.8, we can see how the spinning reserve requirement attempts to tighten the distribution of EC and ENS in comparison to the M-ED (middle plot), whereas the envelope requirement further tightens the distribution in comparison to SR-ED (bottom plot). The tightness of the histogram, implies how well each economic dispatch approach is capable of mitigating system imbalances and thus maintaining power balance as dispatch operations move forward in time.

Hence, there are advantages in properly capturing the underlying system net load variability envelope through its systematic encapsulation. The first main advantage lies with the reductions in the expected values of ENS, EC and their corresponding variances. Secondly, we have to emphasize that this has been achieved at the price of very little computational cost increases, in comparison to a dispatch problem formulation based on 
multi-scenario stochastic optimization.

Table 2.2 Expected Energy Served (EES) by Resources (MWh)

\begin{tabular}{ccrrrr}
\hline & M-ED & \multicolumn{2}{c}{ SR-ED } & \multicolumn{2}{c}{ FE-RHED } \\
\cline { 2 - 6 } & (benchmark) & & Change & & Change \\
\hline 1 & 8.26 & 6.97 & $-15.6 \%$ & 6.49 & $-21.4 \%$ \\
2 & 7.37 & 6.56 & $-11.0 \%$ & 6.26 & $-15.1 \%$ \\
3 & 5.99 & 6.00 & $0.3 \%$ & 6.01 & $0.4 \%$ \\
4 & 4.61 & 5.45 & $18.2 \%$ & 5.75 & $24.8 \%$ \\
5 & 3.79 & 5.04 & $33.1 \%$ & 5.52 & $45.8 \%$ \\
\hline Expected & & & & & \\
Dispatch & 1567 & 1702 & $+8.6 \%$ & 1753 & $+11.9 \%$ \\
Cost (\$) & & & & & \\
\hline
\end{tabular}

Table 2.3 Expected Energy Not Served (EENS), Expected Energy Curtailed (EEC), Expected Net-Load Energy (ENLE) and Total Expected Cost Implications

\begin{tabular}{cccccc}
\hline & M-ED & \multicolumn{2}{c}{ SR-ED } & \multicolumn{2}{c}{ FE-RHED } \\
\cline { 2 - 6 } & (benchmark) & & Change & & Change \\
\hline EENS (MWh) & 0.73 & 0.37 & $-48.5 \%$ & 0.29 & $-61.0 \%$ \\
EEC (MWh) & 0.74 & 0.39 & $-47.2 \%$ & 0.31 & $-58.6 \%$ \\
ENLE (MWh) & 30.01 & 30.01 & - & 30.01 & - \\
\hline Expected & & & & & \\
$\quad$ Total & 3037 & 2462 & $-18.9 \%$ & 2353 & $-22.5 \%$ \\
Cost $(\$)$ & & & & & \\
\hline
\end{tabular}

\subsection{Comparison to Stochastic Planning}

Scenario-based stochastic planning has been proposed widely as a methodology to address the variability and the uncertainty of RES as part of short-term power system planning, especially for unit commitment; see, for example, $[9,10]$. The advantages of stochastic 

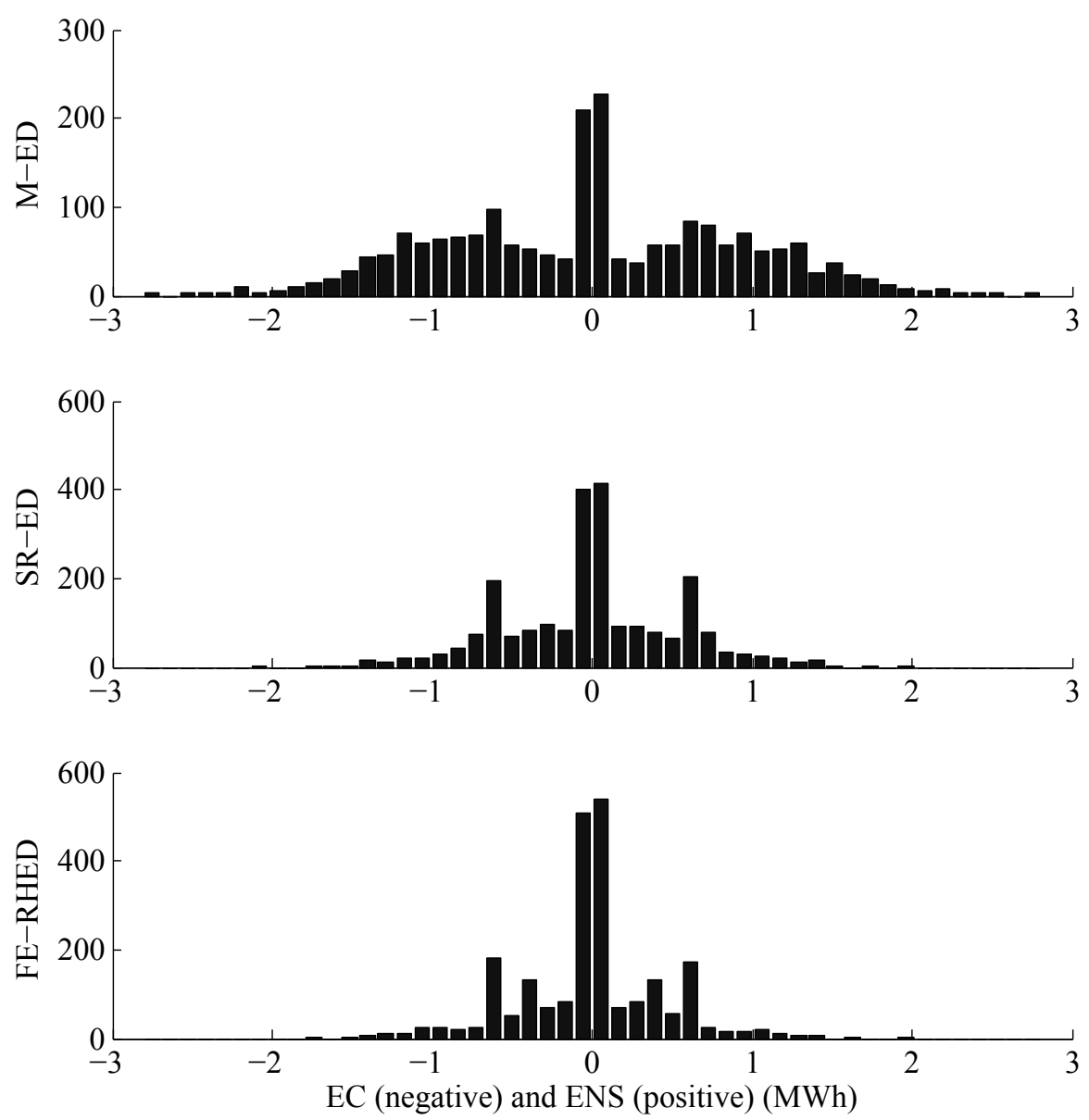

Fig. 2.8 Histograms of EC and ENS for each dispatch approach. 
planning in this respect are many. First, it serves to determine here-and-now generation planning decisions while having foresight of how the system net load may evolve. Typically, this is done with the help of scenario tree. Moreover, stochastic planning approaches may not fully hedged against some extreme low-probability high-consequence events [3]. Lastly, transmission constraints can be handled without much difficulty in stochastic planning approaches [55, 56].

On the other hand, stochastic planning has significant challenges in performing generation scheduling under uncertain and variable RES. Its first challenge pertains to the construction of adequate scenario trees to represent credibly the possible time evolution of the net load $[10,57]$. In order to obtain accurate representations, one has to have a large number of scenarios which, in turn, increases the size of the problems to solve. This is compounded with the fact one needs to define variables and constraints for each possible realization of the scenario tree. Therefore, stochastic planning formulations are yet to emerge as viable practical industry solutions.

The approach we propose based on flexibility envelopes circumvents those dimensional hurdles by ignoring the different likelihood of specific net load trajectories enclosed within the flexibility requirement envelope. All possible net load trajectories enclosed by the envelope are treated equally, and only those at the boundary matter to the generation planner. Moreover, it is much simpler to obtain flexibility requirement envelopes than to build good scenario trees because all that is needed to determine a flexibility requirement envelope is historical/forecast error information. At the same time envelope-based planning is much less computationally intensive than stochastic planning because it is not necessary to carry variables and constraints which apply to all net load scenarios contained within the envelope. We believe that the current approach can be readily adapted to include transmission limits. This, however, remains out of the scope of the dissertation.

\subsection{Summary}

Power system flexibility is an emerging concept there to complement traditional capacity adequacy planning in modern power systems. Flexibility adequacy emphasizes on timely delivery of reserve capacity as much as it emphasizes on the capacity itself. However, current operational and planning practices do not address satisfactorily flexibility requirement and typically assume sufficient ramp-ability of allocated reserve capacity. This can have 
great impact on short- and long-term operations planning when significant amounts of nondispatchable generation is present. In this dissertation, we emphasize the need to model the intra-hourly flexibility potential dynamics of power system resources through the notion of flexibility envelopes. We presented a case study illustrating the value of the flexibility envelope concept in the context of a receding horizon economic dispatch with highly-variable generation. The dispatch approach based on flexibility envelopes, as expected, can decrease energy not served and energy curtailed in comparison to more traditional approaches. Its computational complexity is well under that of other approaches which consider an explicit representation of generation variability and uncertainty (e.g. stochastic optimization, dynamic programming). 


\section{Chapter 3}

\section{Energy-Centric Planning with Flexibility Envelopes}

Currently, power system operations planning practices are undergoing various transformations in an attempt to integrate efficiently significant amounts of sustainable low-carbon power generation technologies. At the heart of this efficient integration lies the need to plan for and exploit the available flexibility in power systems. Flexibility is a relatively new concept addressing the issue of increased variability and uncertainty in power systems admitting high penetration levels of renewable energy sources. To that end, we previously introduced the concept of flexible power envelopes to capture flexibility requirements and flexibility availability from power system resources in terms of time-constrained power deployment. Here, we extend this concept to flexible energy envelopes, thus giving rise to the notion of flexibility assessment in terms of time-constrained energy deployment. This approach is more appropriate for considering flexibility resources like energy storage and demand response. We demonstrate the advantages of planning with flexible energy envelopes with a receding-horizon economic dispatch example integrating energy storage assets.

\subsection{Introduction}

The recently emerging concept of power system flexibility $[5,4]$ is an attempt at modernizing the traditional operating reserve paradigm. The traditional reserve paradigm is mostly concerned with capacity planning, while assuming ample ramping capability of resources. At best, ramping is planned for the 10 (primary/secondary) and 60-minute (tertiary) reserve 
requirements. On the other hand, power system flexibility puts as much emphasis on ramping as it does on capacity planning. It ensures the planned reserve capacity can be deployed (i.e. ramped up or down) in due time, to provide frequency control and load following as dictated by the capacity and ramping behavior of the net load, i.e., load less non-dispatchable renewable energy sources (RES). Recently as well, there has been an increasing interest in energy storage and energy-constrained demand response as flexibility providers, thus adding a third equally-important dimension (i.e. energy) to the emerging flexibility concept.

In Chapter 1, we reviewed the state of the art on operational flexibility, which focused on assessing capacity and ramping capability of resources, while assuming no constraints on energy. To summarize, quantifying flexibility requirements, in the form of flexible operating reserve, has been addressed in recent literature on three main frontiers. First, earlier literature emerged to quantify flexibility requirements with respect to the current industry-based categorization of reserve types: primary/instantaneous, secondary/fast, and tertiary/slow $[35,36,13,37]$. The main emphasis was on optimizing flexibility requirements statistically [14, 39], stochastically [9], reliability-wise [35, 40, 17, 41], and via cost/benefit analysis $[42,27,41]$. Although these methods fell short of addressing the complete intrahourly spectrum of flexibility requirements, they still provided a valuable set of tools that could be extended to the remaining intra-hourly durations. Second, more recent literature addressed the full spectrum of intra-hourly flexibility requirements by statistical analysis of step changes applied to the net load time series for a range of intra-hourly durations. The more notable approaches, which also incorporated intra-hourly flexibility in scheduling mechanisms, are the proposed flexibility envelope in Chapter 2, the flying brick in [22], and the probability box in [55]. Third, stochastic-based methodologies, surveyed in [9], managed operating reserve implicitly via the power balance imposed on the stochastic scenarios. Stochastic-based approaches, however, suffer from the curse of dimensionality rendering them computationally intractable even for tertiary reserve management.

For the most part, the state of the art catered to traditional power systems that are dominated by thermal generation. In contrast, sustainable power systems are increasingly admitting various energy-constrained flexible resources, to help mitigate the impact of variability and uncertainty arising from higher penetration levels of RES. The two main types are energy storage and demand response devices, which are capable of providing ample ramping (i.e. quasi-instantaneous on/off switching) but limited energy capacity. Thus, we 
argue here that it is also beneficial to address the concept of power system flexibility from an energy-centric perspective, to explicitly manage energy deployment of these types of resources. To this end, we extend model in Chapter 2 to managing energy flexibility in lieu of power flexibility.

In Chapter 2, we proposed the concept of flexibility requirement envelopes to capture the capacity and ramping requirements over some operational time, as dictated by net load variability and uncertainty. Likewise, we used envelopes to constrain flexibility deployment by resources in terms of their capacity and ramping limitations. The approach was that, to provide adequate system flexibility, dispatch levels of flexibility resources have to be prepositioned appropriately such that their aggregate flexible power envelope encloses the flexible power requirement envelope arising from the variable and uncertain net load. This ensures resources have sufficient capacity and ramping capability to cover the capacity and ramping requirements arising from the net load.

On the other hand, the inclusion of energy-constrained devices in planning would require prepositioning their energy state levels, rather than their power dispatch levels. Therefore, here we reconsider the flexibility envelope approach from an energy standpoint. In other words, we argue in favor of replacing the flexible power requirement envelope by the flexible energy requirement envelope, while we also replace the flexible power envelopes of resources by their flexible energy envelopes. Such envelopes are capable of capturing energy, power, and ramp altogether in a single envelope. This way, the energy states of energy-constrained resources can be prepositioned - along with the power dispatch levels of other conventional resources - such that the aggregate flexible energy envelope of all resources encloses the flexible energy requirement envelope arising from the net load. This poses a paradigmatic shift in the way we look at operating reserve as flexible deployable energy, rather than flexible deployable power.

\subsection{Quantifying Energy Flexibility Requirements}

In Chapter 2, we obtained the flexible power requirement envelope from net load power step changes. The envelope's height and slope determine how much power capacity and ramping, respectively, over the envelope's horizon are needed to cover appropriately plausible net load variability and uncertainty. This idea is re-iterated here followed by its extension to calculating the flexible energy requirement envelope. We will show two approaches to 
obtaining the flexible energy requirement envelope. One approach first integrates the net load power step changes to obtain the net load energy step changes. Subsequently, the flexible energy requirement envelope is calculated statistically from the aforementioned net load energy step changes, by adapting the procedure from Chapter 2. The second approach obtains the flexible energy requirement envelope directly, by integrating the flexible power requirement envelope from Chapter 2.

\subsubsection{The Flexible Power Requirement Envelope}

The net load measurement at present time $k$ is used as a constant forecast (reference) level for the upcoming short-term operational horizon. This is referred to as a persistence forecast and is believed to be the most accurate under high penetration of renewable energy sources [58]. More importantly, it is by deviating from the present net load level (i.e. the persistence forecast) that a need to deploy flexibility over the operational horizon arises - at least from our perspective in this dissertation.

Consider a net load power time series $\ell(k), k=1, \ldots, K$. From $\ell(k)$, we can obtain the step change in the net load at time $\tau$ as seen from the present time $k$

$$
\Delta P(\tau ; k)=\ell(k+\tau)-\ell(k)
$$

which represents deviation from the persistence forecast $\ell(k), \tau$ units of time later. By fixing $\tau$ and sweeping through $k$, we obtain $k$ realizations of the net load step change $\Delta P(\tau ; k)$ as a function of $\tau$ only. These realizations can then be used to obtain the standard deviation $\sigma_{P}(\tau)$ as a function of $\tau$, as well. From this, the flexible power requirement envelope can be defined as a multiple of the standard deviation $\sigma_{P}(\tau)$, depending on the desired percentile coverage of flexibility requirements. For example, if the step change realizations at $\tau$ are fitted to a Laplace distribution [51], multiplying $\sigma_{P}(\tau)$ by a factor of 1.63 covers the $90^{t h}$ percentile of all plausible net load power deviations, $\tau$ units of time later. The decision maker must choose the appropriate multiplication factor, to cover the desired percentile of the distribution.

For example, consider the net load time series obtained for the Bonneville Power Authority for the year 2014 [59], which contains roughly 89000 data points. The sampling period is $T=5$ minutes, and the operational horizon is set to one hour (i.e., 12 sub-hourly time steps). The method outlined above is applied to all $\tau=m T$, where $m=1, \ldots, 12$. 
Fig. 3.1 plots the normalized histogram (gray area) of $\Delta P(\tau ; k)$ for $\tau=60$ minutes, which is also fitted to a Laplace distribution (red curve). Assuming a Laplace distribution at

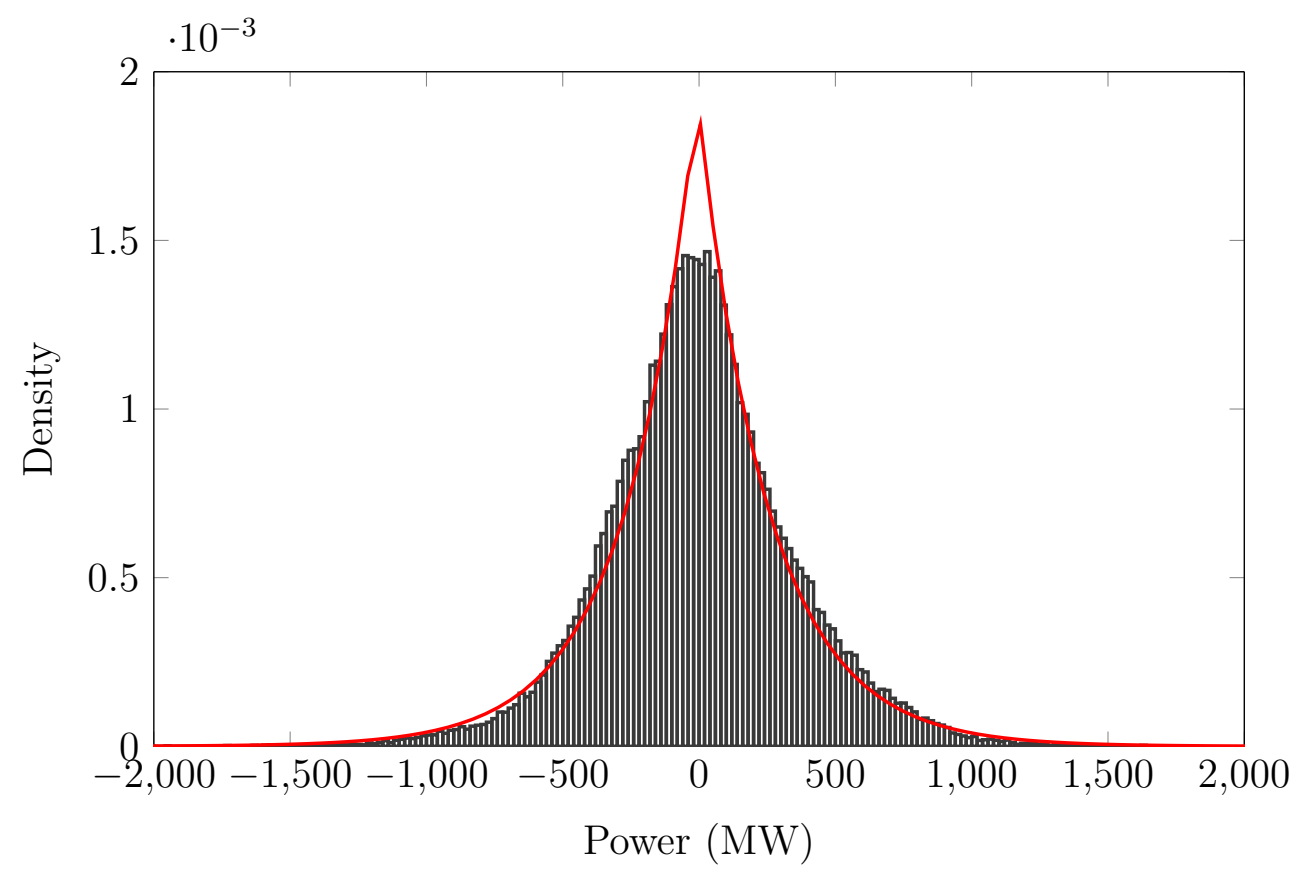

Fig. 3.1 Laplace PDF fitted to power step changes at $\tau=60$ minutes.

every $\tau$ allows us to calculate the standard deviation $\sigma_{P}(\tau)$, as shown in Fig. 3.2. The plot of $\sigma_{P}(\tau)$ resembles the shape of an envelope over the horizon $\tau=[0,60]$ minutes, as desired. Fig. 3.3 reinforces the concept visually by ploting several envelopes for integer multiples of the standard deviation $\sigma_{P}(\tau)$.

Last, the choice of the Laplace distribution is common in the literature for modeling wind variability. The gap between the histogram and the fitted Laplace distribution (red curve) at the center may be an artifact of the BPA dataset. This technical nuance is beyond the scope of the dissertation. What matters ultimately is obtaining the flexibility requirement envelope by any suitable mean, to perform operational planning via the flexibility envelopes framework. 


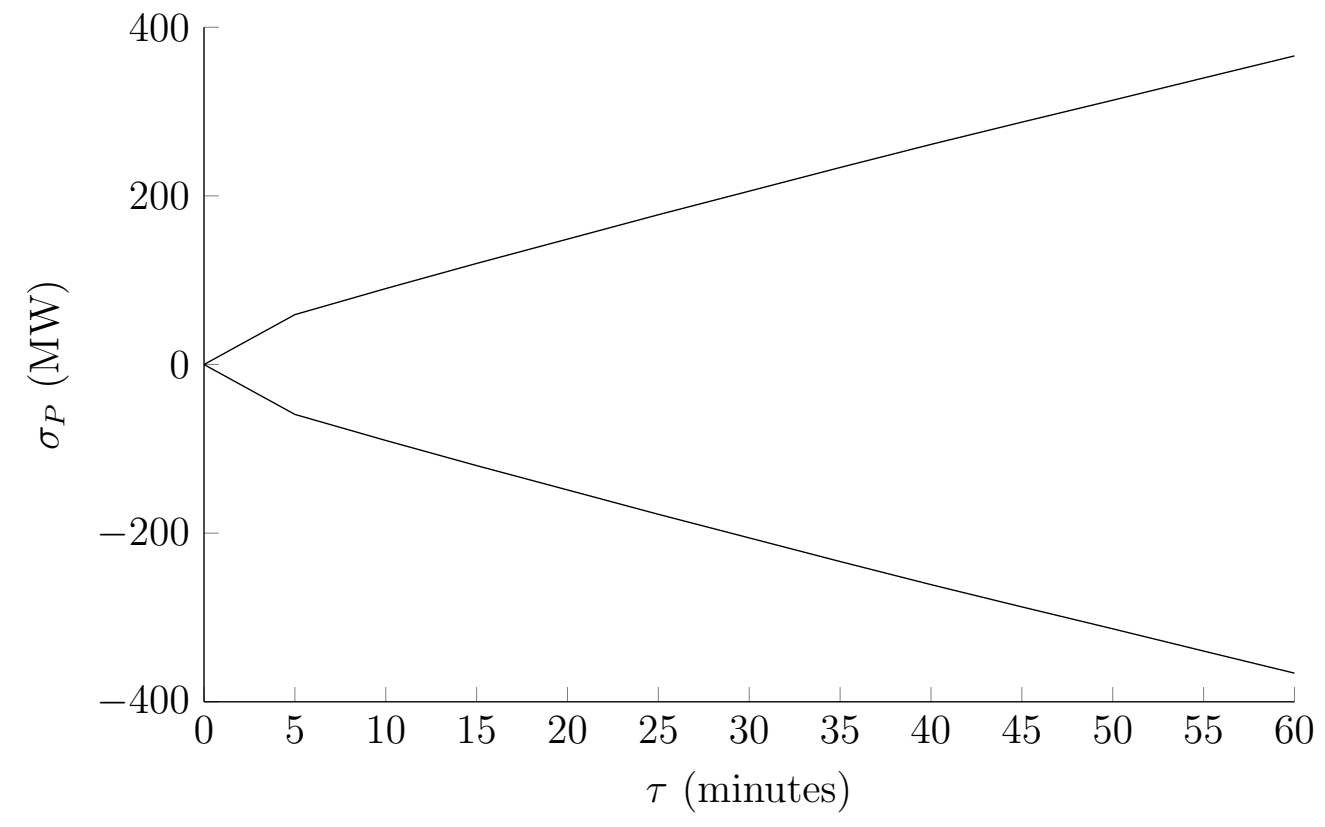

Fig. 3.2 Standard deviation of power step changes as a function of $\tau$.

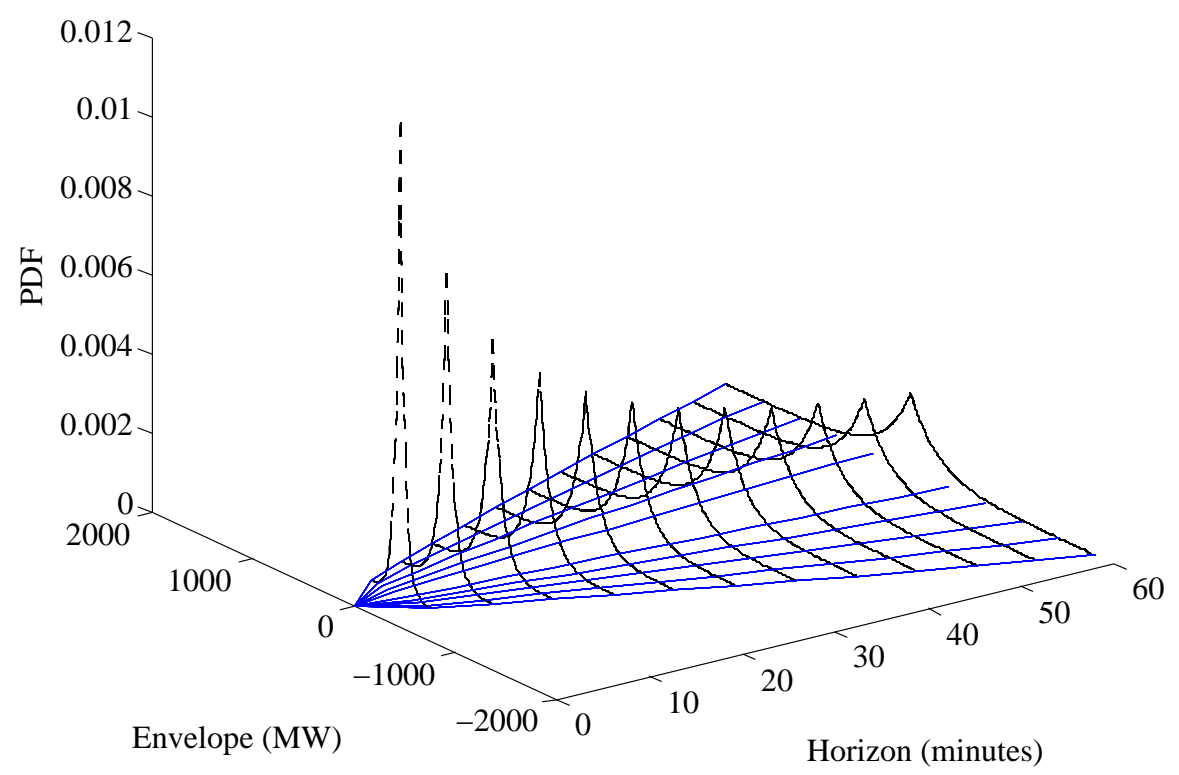

Fig. 3.3 Flexible power requirement envelopes plotted as integer multiples of the standard deviation $\sigma_{P}$. 


\subsubsection{The Flexible Energy Requirement Envelope-First Approach}

First, we integrate the net load power step change (3.1) to obtain the net load energy step change $\Delta E(\tau ; k)$

$$
\Delta E(\tau ; k)=T \sum_{i=1}^{\tau / T} \ell(k+i)-\tau \ell(k)
$$

$\Delta E(\tau ; k)$ represents the energy deviation in the net load $\tau$ units of time later, as seen from the present time $k$. Next, following the methodology in Section 3.2.1, we can obtain the energy standard deviation $\sigma_{E}(\tau)$ as a function of $\tau$, from which we obtain the flexible energy requirement envelope by multiplying $\sigma_{E}(\tau)$ by a pre-determined factor.

Using the same net load time series from [59], Fig. 3.4 plots the normalized histogram (gray area) of $\Delta E(\tau ; k)$ for $\tau=60$ minutes, which also fits well to a Laplace distribution (red line). Assuming a Laplace distribution at every $\tau$, Fig. 3.5 plots the standard deviation

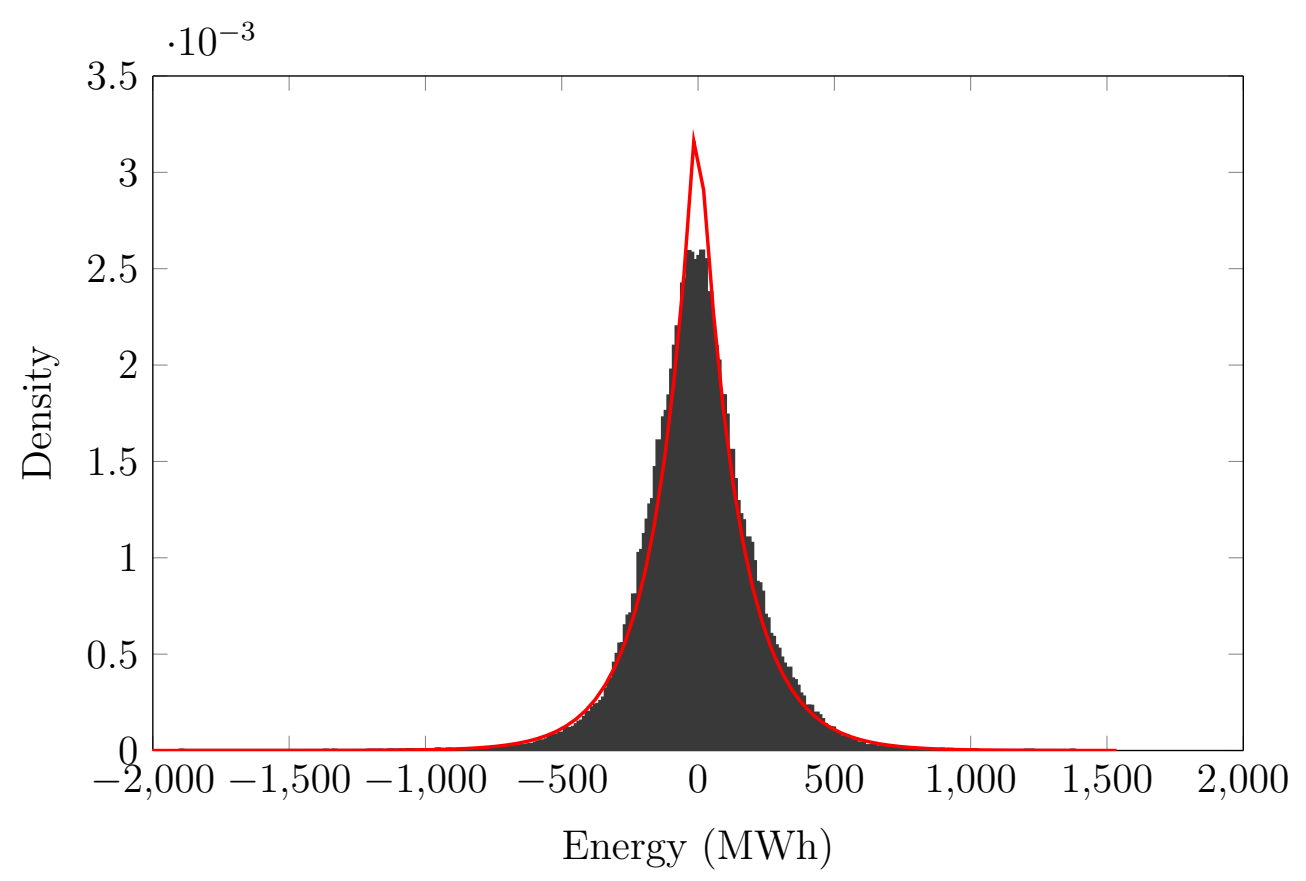

Fig. 3.4 Laplace PDF fitted to energy step changes at $\tau=60$ minutes.

$\sigma_{E}(\tau)$. Likewise, the plot of $\sigma_{E}(\tau)$ resembles the shape of an envelope over the horizon $\tau=[0,60]$ minutes, as expected. 


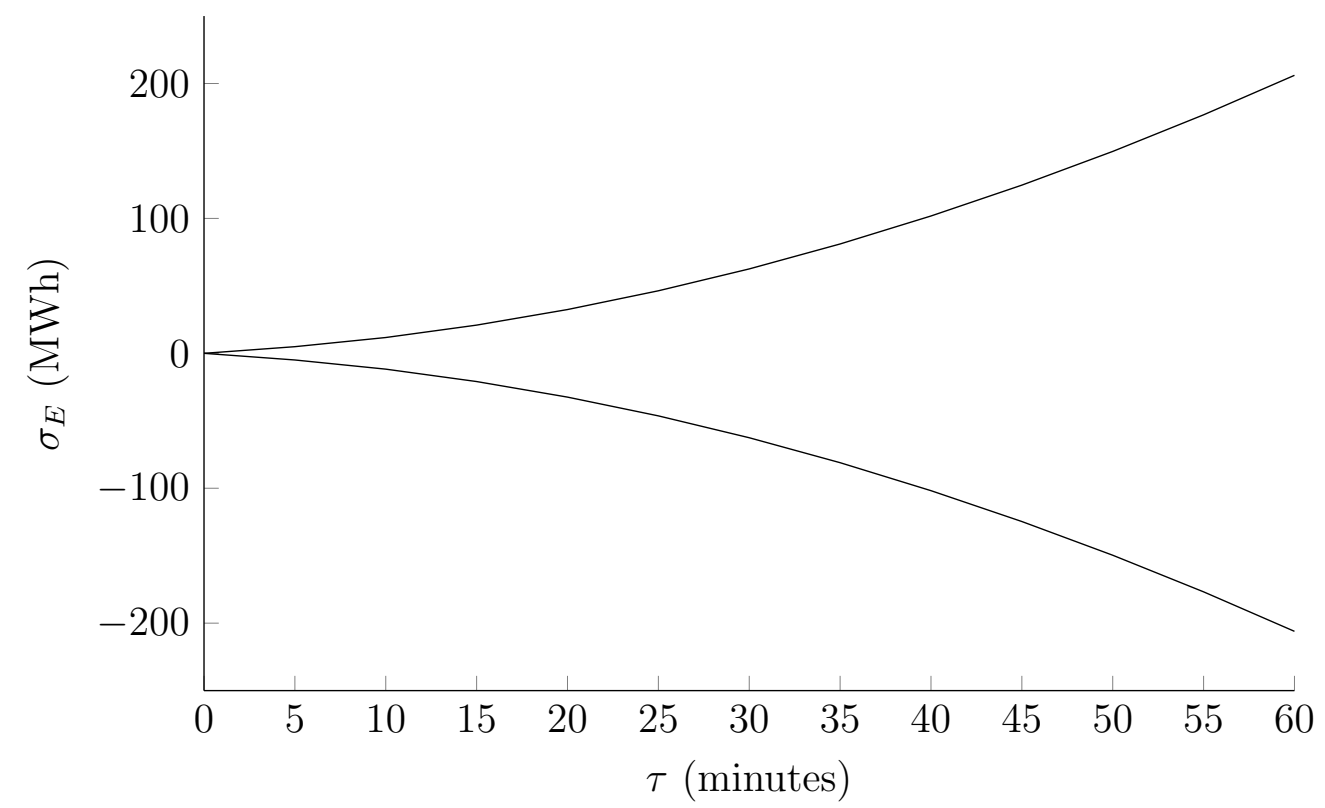

Fig. 3.5 Standard deviation of energy step changes as a function of $\tau$.

\subsubsection{The Flexible Energy Requirement Envelope-Second Approach}

In this case we obtain the flexible energy requirement envelope directly by integrating the original flexible power requirement envelope:

$$
\sigma_{\Sigma P}(\tau)=T \sum_{i=1}^{\tau / T} \sigma_{P}(i)
$$




\subsubsection{Comparison of the Two Approaches}

Next, we compare the flexible energy requirement envelopes obtained by both approaches. To do so, we start by taking the square of both sides of (3.3)

$$
\begin{aligned}
\sigma_{\Sigma P}^{2}(\tau) & =\left(T \sum_{i=1}^{\tau / T} \sigma_{P}(i)\right)^{2} \\
& =T^{2} \sum_{i=1}^{\tau / T} \sigma_{P}^{2}(i)+T^{2} \sum_{j \neq i} \sum_{P}(i) \sigma_{P}(j) \\
& \geq T^{2} \sum_{i=1}^{\tau / T} \sigma_{P}^{2}(i)+T^{2} \sum_{j \neq i} \sum_{P} \sigma_{P}(i, j)
\end{aligned}
$$

through the application of the Cauchy-Schwarz inequality

$$
|\sigma(i, j)| \leq \sqrt{\sigma^{2}(i) \sigma^{2}(j)}
$$

Furthermore, taking the variance of both sides of (3.2) yields

$$
\begin{aligned}
\operatorname{Var}(\Delta E(\tau ; k)) & =\operatorname{Var}\left(T \cdot \sum_{i=1}^{\tau / T} \Delta P(i ; k)\right) \\
& =T^{2} \cdot \sum_{i=1}^{\tau / T} \operatorname{Var}(\Delta P(i ; k)) \\
& +T^{2} \cdot \sum_{i \neq j}^{\tau / T} \operatorname{Cov}(\Delta P(i ; k), \Delta P(j ; k))
\end{aligned}
$$

Replacing $\operatorname{Var}(\Delta E(\tau ; k))$ by $\sigma_{E}^{2}(\tau), \operatorname{Var}(\Delta P(\tau ; k))$ by $\sigma_{P}^{2}(i)$, and $\operatorname{Cov}(\Delta P(i ; k), \Delta P(j ; k))$ by $\sigma_{P}(i, j)$ gives

$$
\sigma_{E}^{2}(\tau)=T^{2} \sum_{i=1}^{\tau / T} \sigma_{P}^{2}(\tau)+T^{2} \sum_{j \neq i} \sum_{P} \sigma_{P}(i, j)
$$


Comparing (3.4) to (3.7), we conclude that

$$
\sigma_{E}^{2}(\tau) \leq \sigma_{\Sigma P}^{2}(\tau)
$$

This result implies that the flexible energy requirement envelope obtained via the first approach, $\sigma_{E}(\tau)$, is less stringent than the one obtained via the second approach, $\sigma_{\Sigma P}(\tau)$. Intuitively, summing of the power step changes to obtain the energy step changes is a form of smoothing (i.e. a moving-average filter). Consequently, one can use the integral of the flexible power requirement envelope, $\sigma_{\Sigma P}(\tau)$, as an upper bound on the flexible energy requirement envelope $\sigma_{E}(\tau)$.

For the same BPA data as above, Fig. 3.6 plots both envelopes, which empirically confirms this upper bound relationship. It is a matter of perspective or preference as to which envelope to consider for planning.

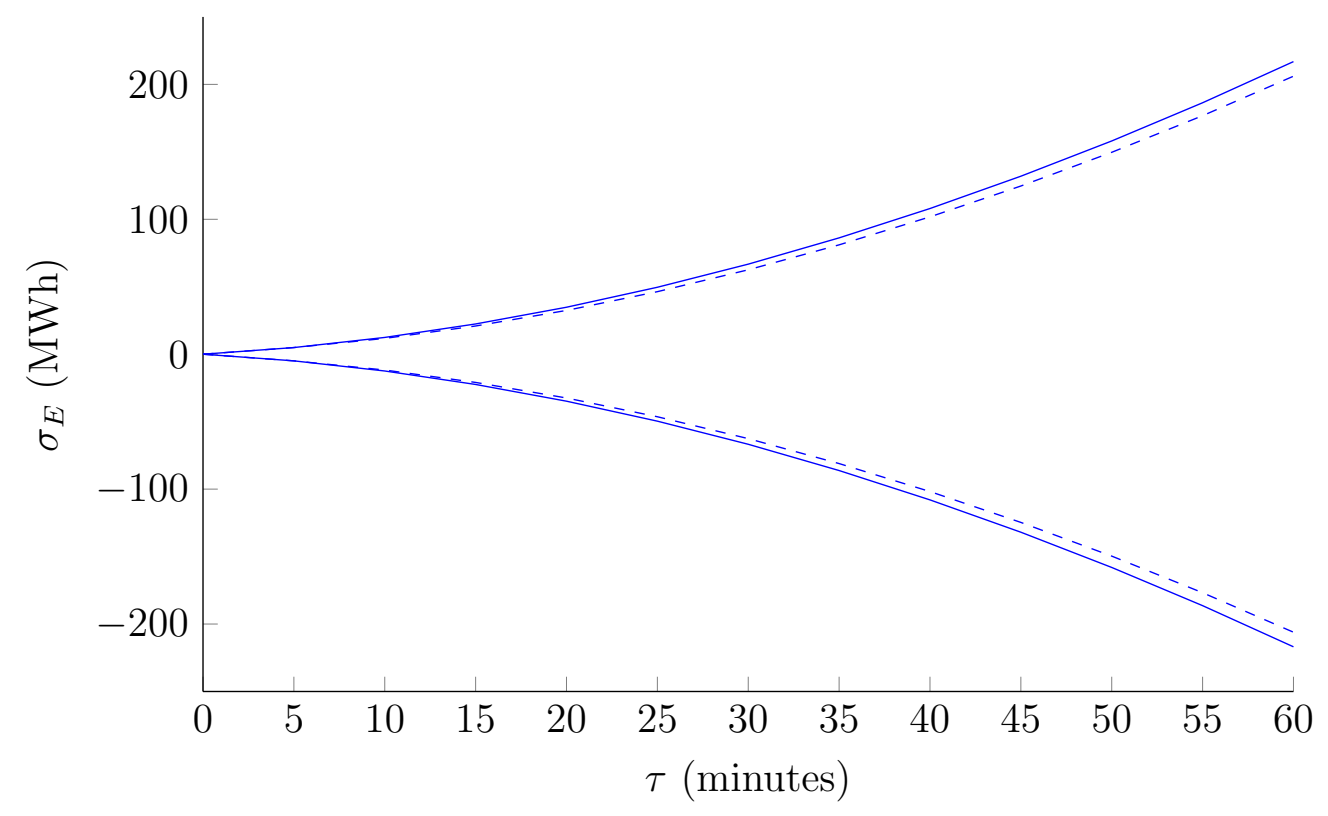

Fig. 3.6 $\sigma_{E}$ (dashed) compared to $\sigma_{\Sigma P}(\tau)$ (solid).

\subsection{The Flexible Energy Envelope of Power System Resources}

In line with the previous section, a planned deviation from the present (scheduled) power level of a power system resource is what constitutes flexibility (power) deployment. In the 
context of this chapter, we now seek to quantify the energy associated with a power system resource when its power output is deviating over the operational horizon. Let $y(k)$ represent the power set point of a resource at present time $k$. The output power can undergo step changes as flexibility is deployed over forward time $\tau$

$$
\Delta P(\tau ; k)=y(k+\tau)-y(k)
$$

For example, consider a thermal generation unit with minimum output level of $10 \mathrm{MW}$, maximum output level of $50 \mathrm{MW}$, and a maximum upward/downward ramp rate of 1 MW/minute. Given an initial output of $30 \mathrm{MW}$, Fig. 3.7 plots the flexible power envelope of the thermal generation unit. In contrast, consider a lossless energy storage device that

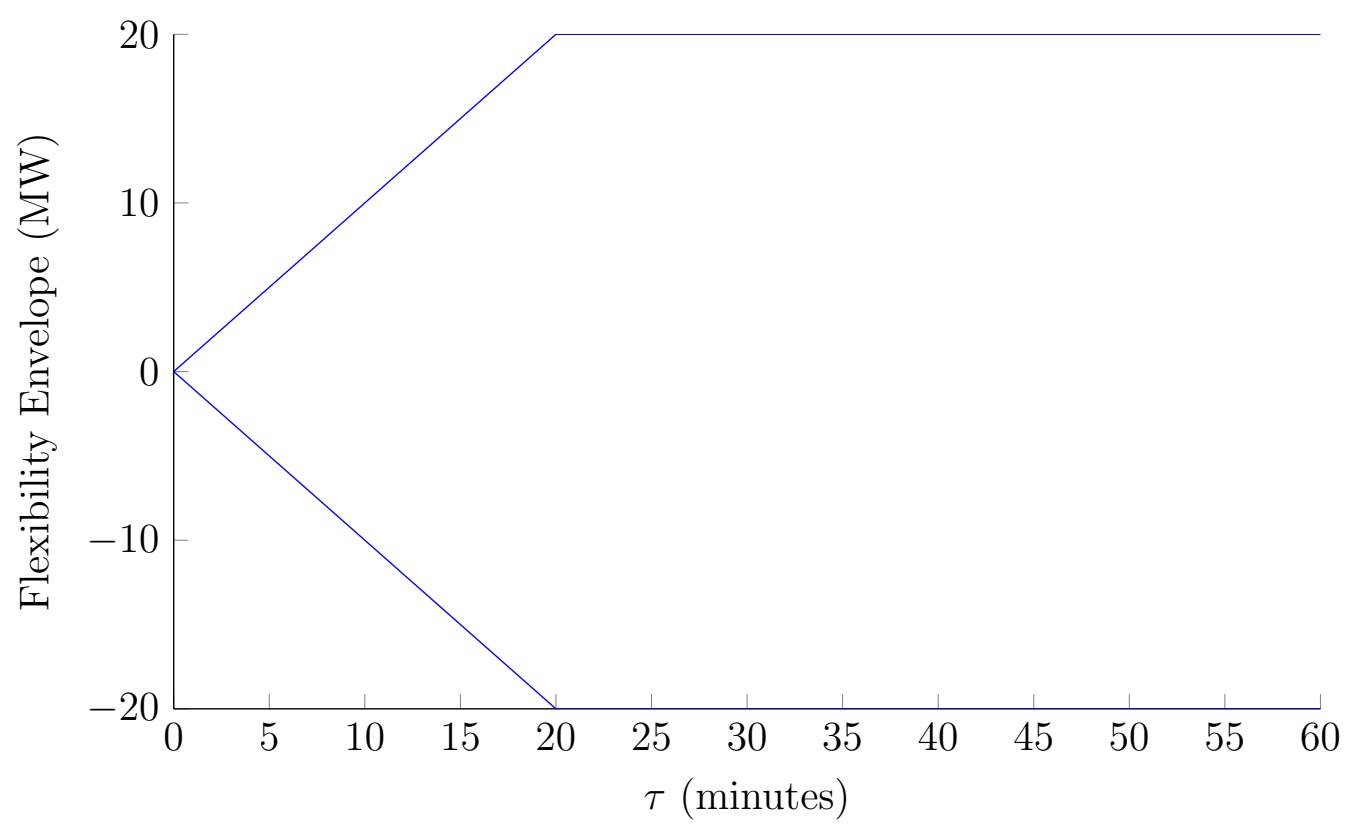

Fig. 3.7 Sample power envelope of a thermal generation unit.

can deliver or absorb energy at a rated power of $10 \mathrm{MW}$ and can hold up to $10 \mathrm{MWh}$ of energy. Given an initial state of charge of $5 \mathrm{MWh}$, Fig. 3.8 plots the flexible power envelope of the lossless energy storage asset. In both examples, the sampling period $T$ is 5 minutes and $\tau$ varies from 0 to 60 minutes. Integrating (3.9) yields energy step change at $\tau$ : 


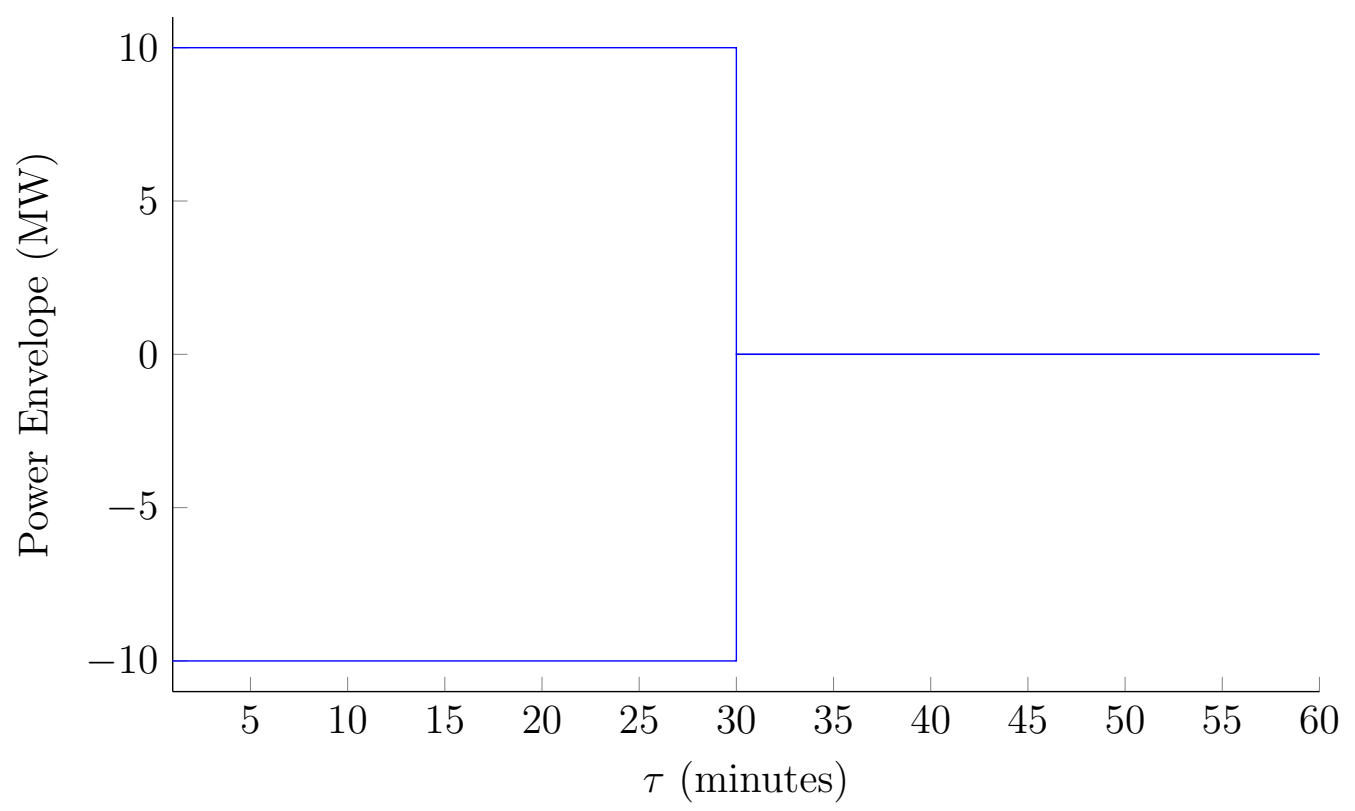

Fig. 3.8 Sample power envelope of an energy storage unit.

$$
\Delta E(\tau ; k)=T \sum_{i=1}^{\tau / T} y(k+i)-\tau y(k)
$$

where this quantity represents the amount of energy a resource can deliver by $\tau$ units of time later, as seen from the present time $k$.

Fig. 3.9 plots the flexible energy envelope of the thermal generation unit, given $y(k)=30$ MW, and Fig. 3.10 plots the flexible energy envelope of the lossless energy storage for the initial state of charge of $5 \mathrm{MWh}$. In the case of the storage unit, the rising/falling slopes of the energy envelope is its rated power of $10 \mathrm{MW}$. Moreover, the envelope is set to saturate at $\tau=30$ minutes, due to depletion of upward or downward energy flexibilityi.e. the energy store is either fully charged or fully discharged, respectively. In contrast, the flexible energy envelope of the thermal generation unit continues to ramp up/down, albeit at different rates, once it reaches its maximum and minimum power output levels.

The key observation here is that the flexible power envelope of the storage unit is not the most appropriate to perform operational flexibility planning. As it stands, the flexible power envelope in Fig. 3.8 is myopic in the sense that it neglects the fact that the storage asset could provide flexibility well beyond the time where its state of charge limits are 


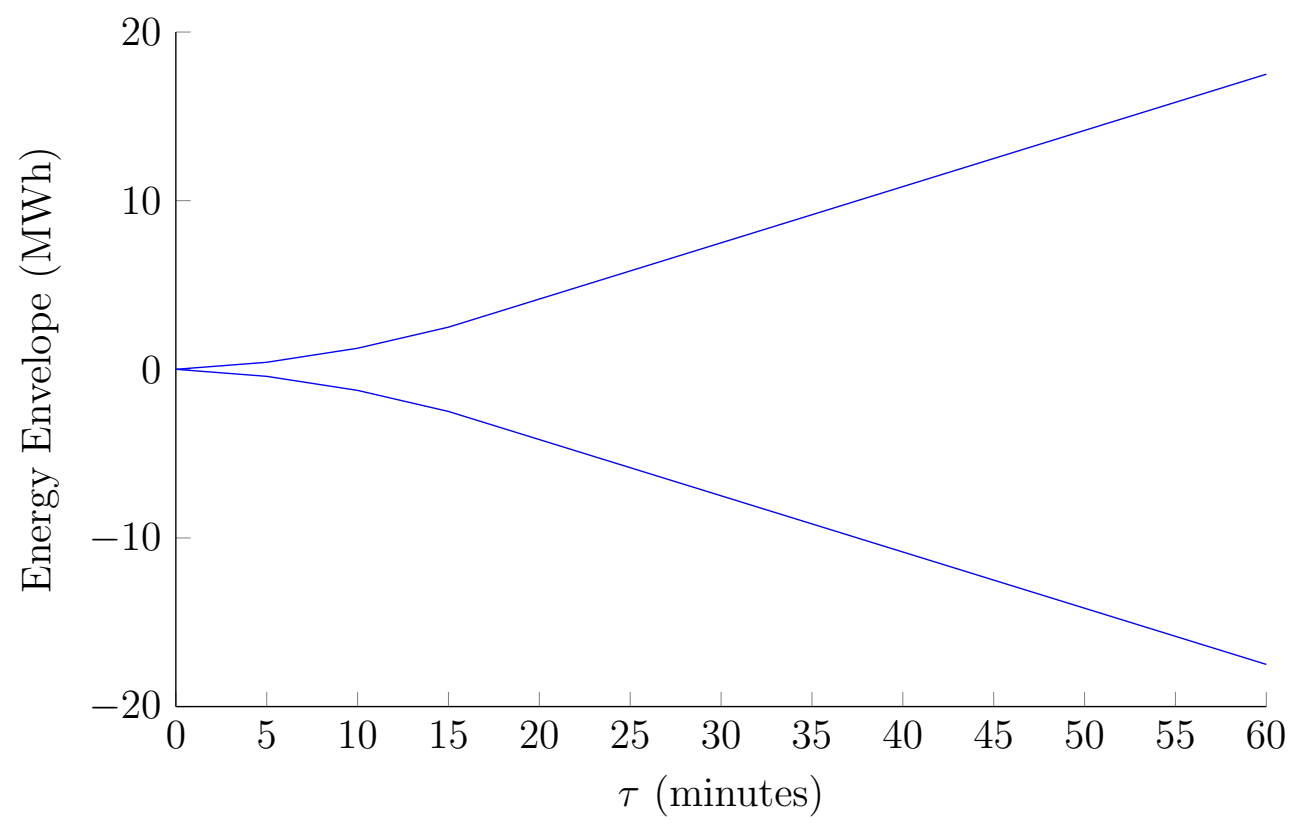

Fig. 3.9 Energy envelope of the thermal generation unit as a function of $\tau$.

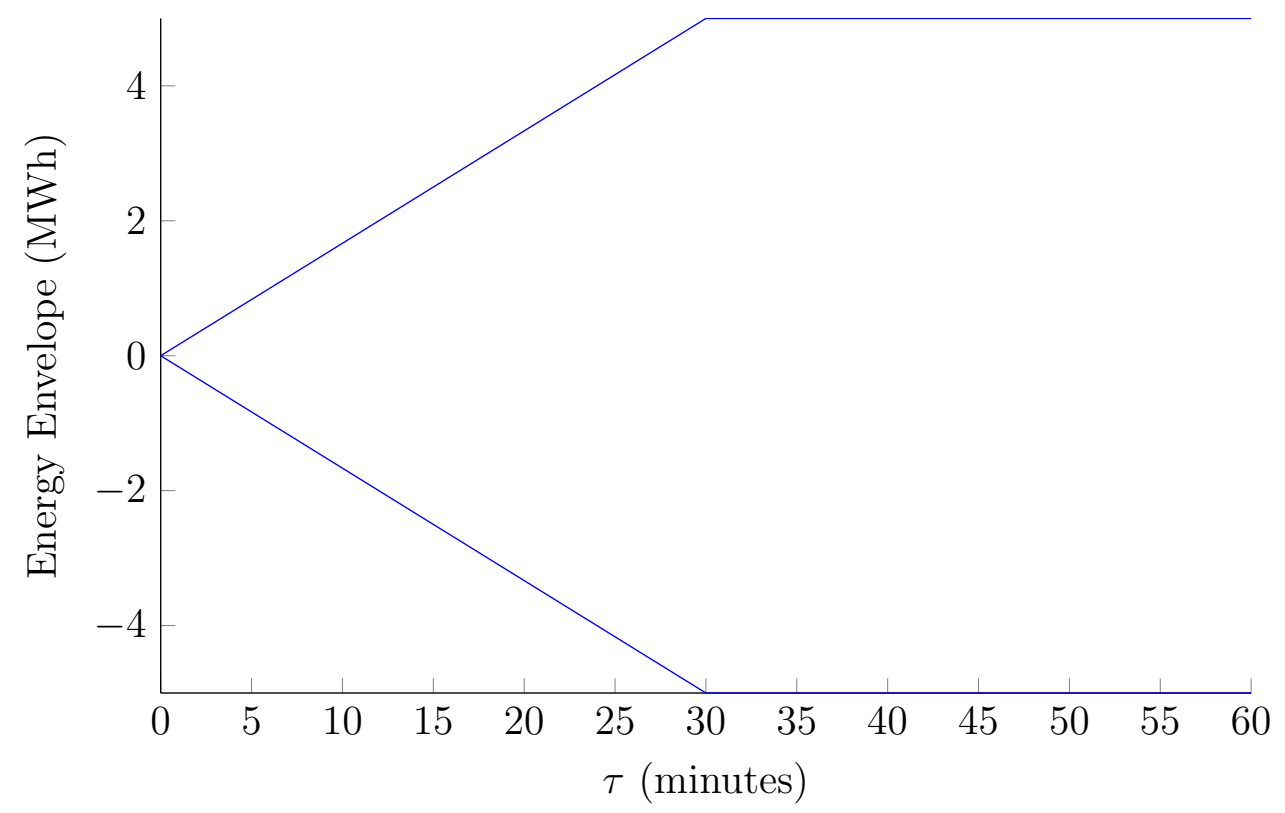

Fig. 3.10 Energy envelope of the energy storage unit as a function of $\tau$. 
reached. When one turns to the flexible energy envelope of the storage asset in Fig. 3.10, it is clear that the corresponding envelope is such that the storage unit state of charge can evolve in a range well beyond the time at which state of charge limits are reached for rated power charging/discharging. In fact, one sees readily that the shape of the flexible energy envelope of the storage unit is similar to the flexible power envelope of the generating unit. It has been shown in Chapter 2 that this envelope is appropriate to conduct forwardlooking flexibility planning. By using flexible energy envelopes, power system operators could break away from the myopic character of flexible power envelopes associated with energy-limited resources. In addition, energy envelopes are still consistent for modeling the flexibility of conventional generation. Therefore, energy-based flexibility planning, being more resource-inclusive than power-based flexibility planning, should have more potential at leveraging the available flexibility in a power system.

We show next how flexible energy envelopes can provide an appropriate operations planning mechanism to best exploit the flexibility of storage or other energy-limited assets. Flexible energy envelopes allow for simultaneous asset state of charge and power output positioning, something not possible with flexible power envelopes.

\subsection{Energy-Centric Planning with Envelopes}

Planning with flexible power envelopes has already been proposed in Chapter 2. Here, however, the use of flexible energy envelopes will allow us to preposition energy states of energy-constrained resources, to optimize their deployment moving forward. The following example formulates a mixed-integer linear program integrating energy storage in a recedinghorizon economic dispatch problem. Two energy storage strategies will be compared. The first strategy deploys energy storage resources myopically without planning their potential use moving forward - i.e., their envelopes are not aggregated with the remaining resources. The second strategy deploys energy storage assets anticipatorily, by planning their use according to their flexible energy envelopes - i.e., their envelopes are aggregated with the remaining resources. The two strategies are benchmarked against a case without storage to compare their relative performance. 


\subsubsection{Problem Setup}

Receding Horizon Operation: As illustrated by Fig. 3.11, the power system is running during the $h^{\text {th }}$ hour, at the $k^{\text {th }}$ economic dispatch step. The decision maker must redispatch the committed resources to match the new forecast net load $\ell(k)$ by ramping these resources from their previous output levels at step $k-1$ subject to ramping constraints. Concurrently, the decision maker must preposition these resources at step $k$, such that their projected aggregate flexibility envelope encloses the flexibility requirement envelope over the upcoming operational horizon $k+m$, where $m=1, \ldots, M$ is the receding horizon. In this example, we assume there are 12 economic dispatch steps $k$ within the unit commitment hour $h$, such that the sampling period is $T=5$ minutes and $k=1, \ldots, 12$. The projected horizon of the envelopes is set to be one hour as well, such that $M=12$ and $m=1, \ldots, 12$ (equivalently, $\tau=5, \ldots, 60$ minutes).

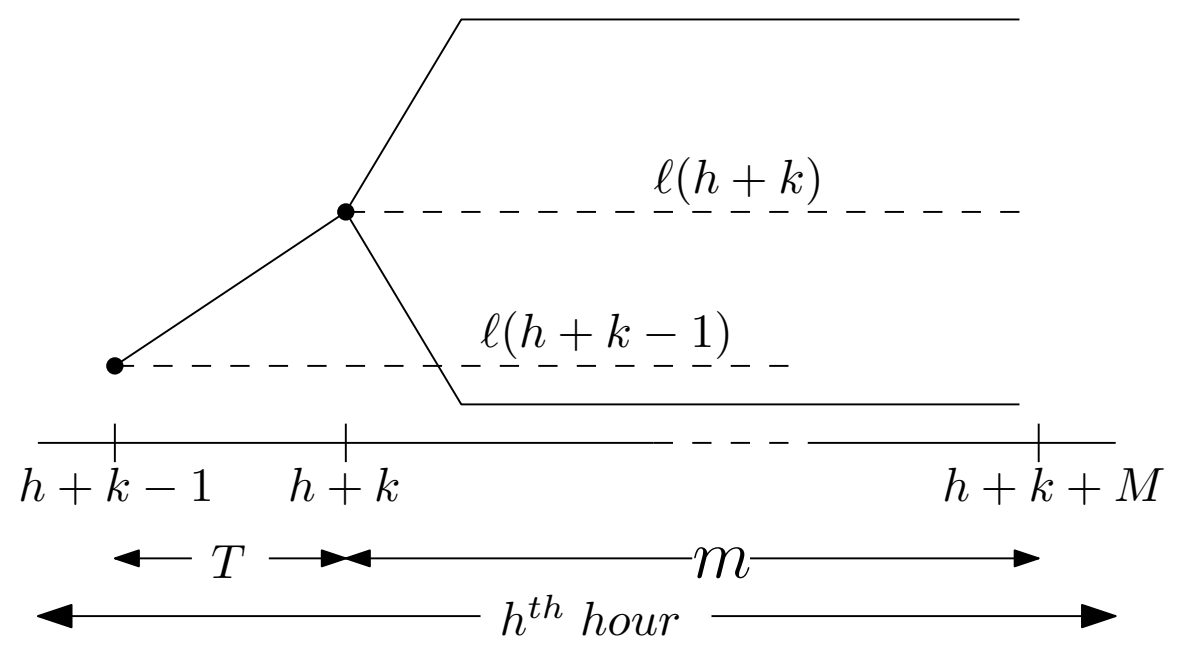

Fig. 3.11 Schematic illustration of a single decision making epoch.

Net Load Model: A series of twelve 5-minute step changes, $\{w(k)\}_{k=1}^{12}$, is generated randomly from a symmetric Laplace distribution that is characterized by $\beta=1 \mathrm{MW} /$ minute. The random step changes are used to update the net load at each economic dispatch step $k$. The step changes are assumed to be independent and identically distributed (i.i.d.), while $\sqrt{2} \beta$ is the standard deviation, and $1.63 \sqrt{2} \beta$ is the $90^{\text {th }}$ percentile for the Laplace distribution. A cap is placed on the realizations of $w(k)$ such that

$$
w(k) \leftarrow \min (1.63 \sqrt{2} \beta, w(k)) ; \quad k=1, \ldots, 12
$$


Taking the initial net load forecast $\ell(1)$ to be $30 \mathrm{MW}$ for economic dispatch step $k=1$, the net load forecasts for the remaining economic dispatch steps are generated by subsequently adding the random step changes $w(k)$. In addition, a cap is placed on the net load maximum and minimum

$$
\begin{array}{ll}
\ell(k+1)=\min (50, \ell(k)+w(k)) ; & k=1, \ldots, 12 \\
\ell(k+1)=\max (10, \ell(k)+w(k)) ; & k=1, \ldots, 12
\end{array}
$$

Fig. 3.12 plots sample realizations of the net load random process bounded by the $90^{\text {th }}$ percentile flexible power requirement envelope.

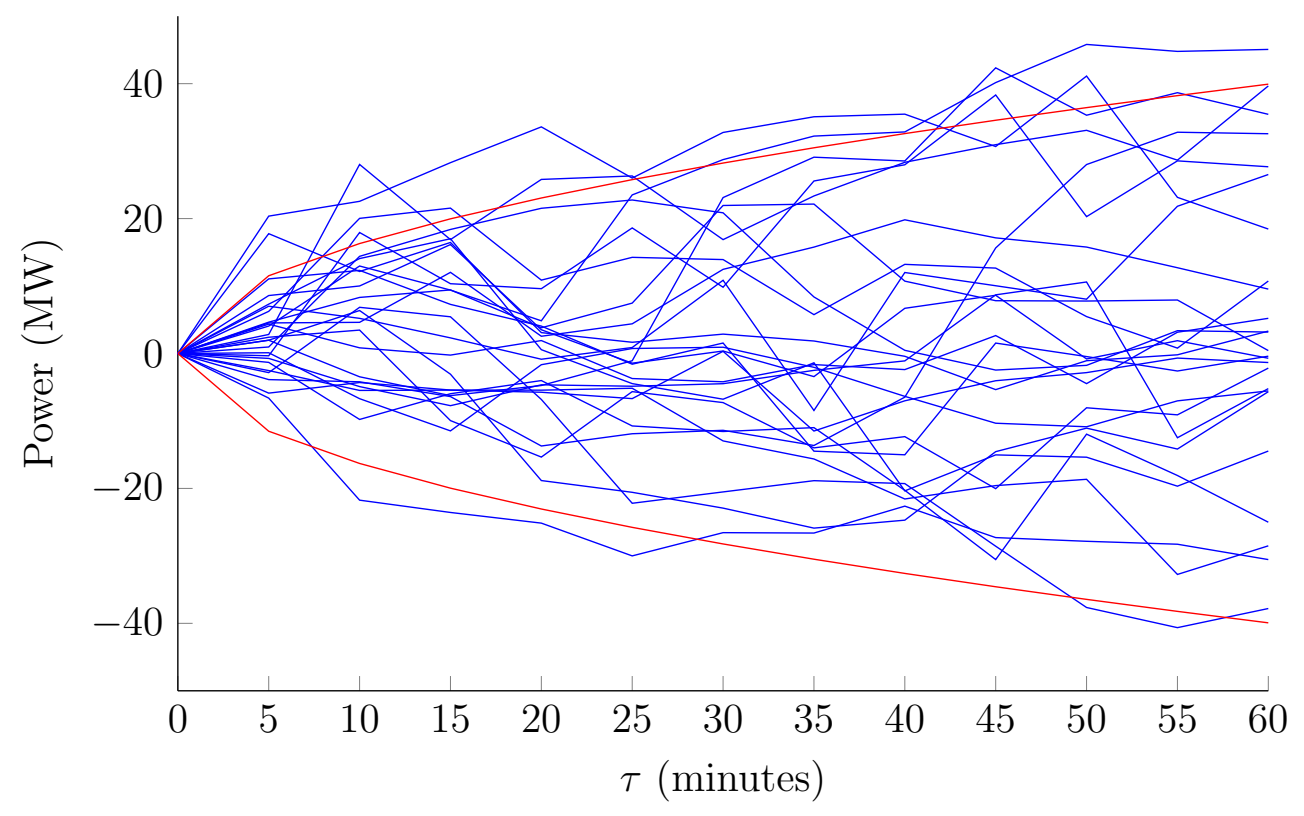

Fig. 3.12 Sample realizations of the net load random process bounded by the $90^{\text {th }}$ percentile flexible power envelope.

The power deviation step changes, as a function of the receding horizon step $m$, are

$$
\Delta P(m)=\sum_{i=1}^{m} w(i) ; \quad m=1, \ldots, 12
$$


while the energy deviation step changes, as a function of $m$, are

$$
\Delta E(m)=5 \sum_{i=1}^{m} \sum_{j=1}^{i} w(j) ; \quad m=1, \ldots, 12
$$

Flexible Power Requirement Envelope: The standard deviation envelope $\sigma_{P}(m)$ is computed by taking the variance of (3.14) and noting the i.i.d. property of $\left\{w_{k}\right\}_{k=1}^{12}{ }^{1}$, thus yielding

$$
\sigma_{P}(m)=\sqrt{m} \sqrt{2} \beta \quad m=1, \ldots, 12
$$

For example, multiplying (3.16) by a factor of \pm 1.63 gives the flexible power requirement envelope covering the $90 \%$ confidence region. As mentioned earlier, establishing the optimal coverage level remains an open research question; we shall not address it formally here. Also, the Laplace distribution is assumed to hold for all $m$, as an approximation of the actual distributions.

Flexible Energy Requirement Envelope: The standard deviation envelope $\sigma_{E}(m)$ is found by taking the variance of both sides of (3.15)

$$
\sigma_{E}(m)=5 \sqrt{2} \beta \sqrt{\frac{m^{3}}{3}+\frac{m^{2}}{2}+\frac{m}{6}} \quad m=1, \ldots, 12
$$

by applying the i.i.d. property of $\left\{w_{m}\right\}_{m=1}^{12}$ and the elementary series sum result

$$
\sum_{i=1}^{m} i^{2}=\frac{m^{3}}{3}+\frac{m^{2}}{2}+\frac{m}{6}
$$

Multiplying (3.17) by \pm 1.63 yields the flexible energy requirement envelope covering the 90\% confidence region. Here again the Laplace distribution is assumed to hold for all $\mathrm{m}$. Fig. 3.13 plots (3.17) along with the integral of (3.16), which confirms the flexible energy envelope is less stringent than the flexible power envelope. Fig. 3.14 plots the integral of the sample scenarios of Fig. 3.12, bounded by the $90^{\text {th }}$ percentile flexible energy requirement envelope.

Flexibility Resources: There are five identical conventional generators, $\mathcal{I}_{g}=\{1,2,3,4,5\}$, constrained by capacity and affine ramp limits, which provide flexibility at a fixed incre-

\footnotetext{
${ }^{1}$ We recall that the covariance of i.i.d. random variables is zero.
} 


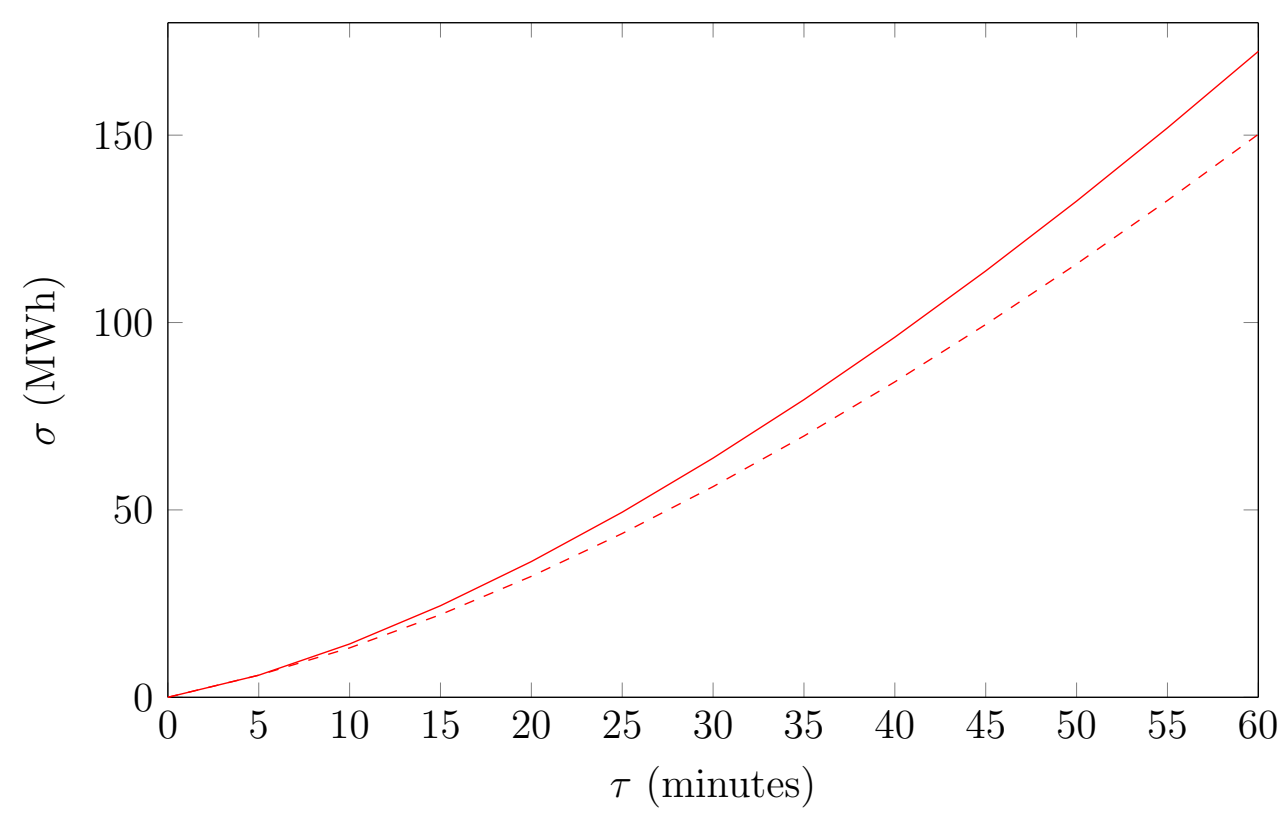

Fig. 3.13 $\sigma_{E}$ (dashed) compared to $\sigma_{\Sigma P}$ (solid).

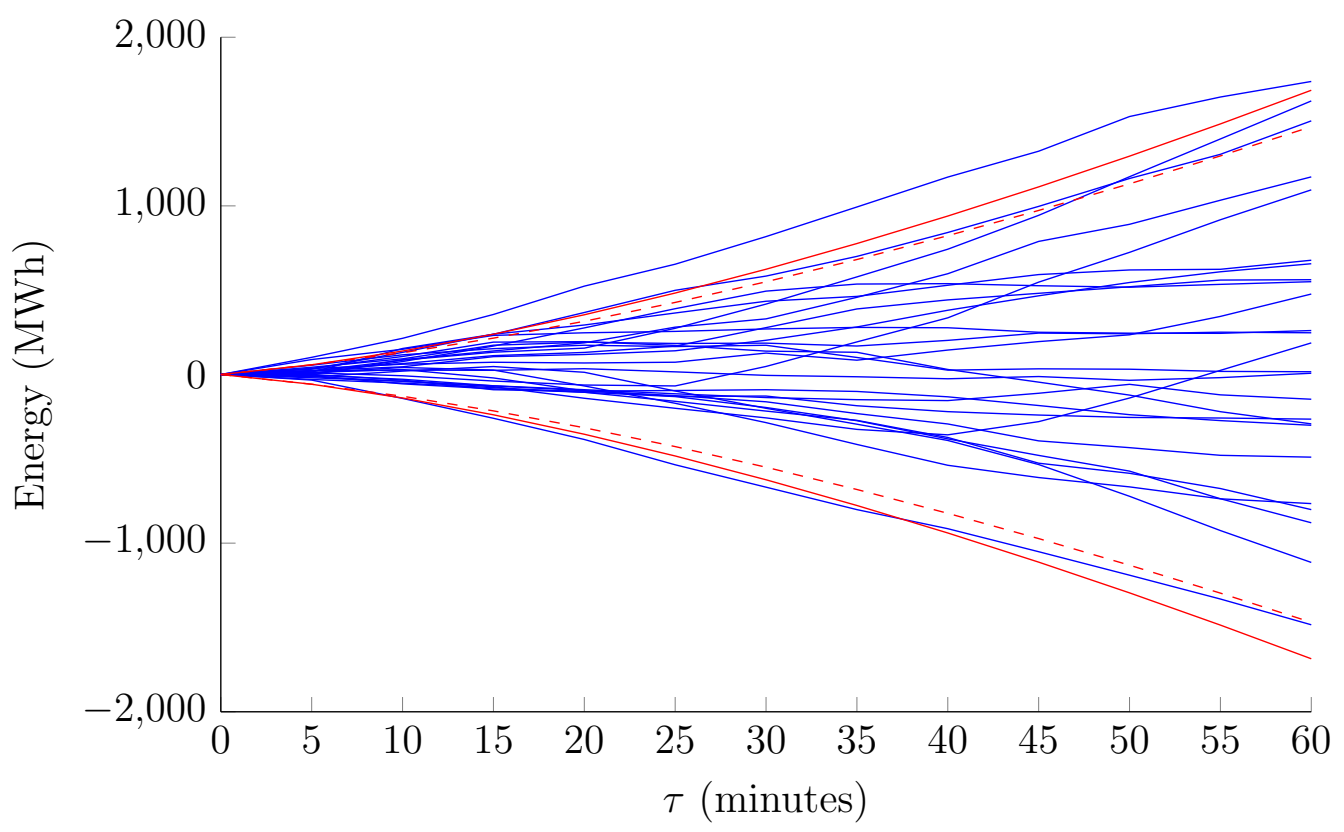

Fig. 3.14 Integral of the sample scenarios in Fig. 3.12, enclosed by the $90^{t h}$ percentile flexible energy requirement envelope. 
mental cost. In addition, two energy storage assets included in the system, $\mathcal{I}_{s}=\{1,2\}$, which do not incur any additional operational costs. Table 3.1 shows the cost and flexibility characteristics of the generators, whereas Table 3.2 shows the flexibility characteristics and efficiencies of energy storage assets. We note that storage asset 1 (the smaller one, energy capacity-wise) has a rated power charge/discharge time of 20 minutes, whereas storage asset 2 (the larger one, energy capacity-wise) has a rated power charge/discharge time of three hours. Clearly, one can see that storage asset 1 is meant for providing intra-hour bridging power, while the second asset is meant primarily for inter-hour energy management.

Table 3.1 Resources' Capacity, Ramping, and Cost Characteristics

\begin{tabular}{ccccc}
\hline $\mathcal{I}_{g}$ & $g^{\max }(\mathrm{MW})$ & $g^{\min }(\mathrm{MW})$ & $R(\mathrm{MW} /$ minute $)$ & $b(\$ / \mathrm{MWh})$ \\
\hline 1 & 10 & 0 & 1.5 & 20 \\
2 & 10 & 0 & 1.5 & 40 \\
3 & 10 & 0 & 1.5 & 60 \\
4 & 10 & 0 & 1.5 & 80 \\
5 & 10 & 0 & 1.5 & 100 \\
\hline
\end{tabular}

Table 3.2 Storage Capacity, Power, and Efficiency Characteristics

\begin{tabular}{cccccc}
\hline $\mathcal{I}_{s}$ & $E^{\max }(\mathrm{MWh})$ & $E^{\min }(\mathrm{MWh})$ & $P(\mathrm{MW})$ & $\mu^{c}$ & $\mu^{d}$ \\
\hline 1 & 25 & 10 & 75 & 0.9 & 0.9 \\
2 & 75 & 10 & 25 & 0.9 & 0.9 \\
\hline
\end{tabular}

Simulations: Five hundred one-hour realizations of the net load random process were generated according to the aforementioned net load model. The no storage case, the myopic storage strategy, and the envelopes-based anticipatory storage strategy were simulated for each of the 500 net load realizations, using the mathematical programs described next.

\subsubsection{Mathematical Program}

The following mixed-integer linear program represents the flexibility envelopes-constrained receding-horizon planning for a single economic dispatch step $k$. To keep the units of measurements consistent, ramp constraints are in $\mathrm{MW} /$ minute, power constraints are in MW, 
and energy constraints are in MWh. This explains the conversion factors $1 / T, 60 / T, T / 60$ throughout the formulation, given that the discretization period is $T=5$ minutes.

Objective: The objective here is to minimize the total dispatch cost of conventional generators. The dispatch objective here assigns no specific operating cost to storage assets as they only serve to shift energy from one time instant to another. ${ }^{2}$

$$
\min \sum_{i \in \mathcal{I}_{g}} b_{i} g_{i}(k)
$$

This is subject to:

Power Balance for Dispatch Step k: Equation (3.20) ensures the power balance at the current dispatch time step $k$.

$$
\begin{gathered}
\sum_{i \in \mathcal{I}_{g}} g_{i}(k)+\frac{60}{T} \sum_{i \in \mathcal{I}_{s}}\left(\mu_{i}^{d}\left[u_{i}(k) s_{i}(k-1)-s_{i}^{\downarrow}(k)\right]\right. \\
\left.+\frac{1}{\mu_{i}^{c}}\left[\left(1-u_{i}(k)\right) s_{i}(k-1)-s_{i}^{\uparrow}(k)\right]\right)=\ell(k)
\end{gathered}
$$

Multiplying by the factor $60 / T$ converts the step changes in storage levels from MWh to MW. The variable $u_{i}$ is binary and indicates the charging status of storage unit $i$, whereas $s_{i}(k-1)$ is the previous storage state of charge at time step $k-1$ and appears here as a parameter. The inclusion of storage efficiencies $-\mu^{c}$ and $\mu^{d}$ for charging and discharging processes, respectively - necessitates representing the storage states by two mutually exclusive variables, $s_{i}^{\uparrow}$ (charge) and $s_{i}^{\downarrow}$ (discharge), because the storage dispatch decision (i.e. whether to charge or discharge at $k$ ) is unknown a priori.

Dispatch for Generator $i \in \mathcal{I}_{g}$ at Step $k$ : These bound the generators by their capacity and ramp rates, respectively, as they ramp from the previous (given) power output level at time step $k-1$.

$$
\begin{gathered}
g_{i}^{\min } \leq g_{i}(k) \leq g_{i}^{\max } \\
-R_{i} \leq \frac{1}{T}\left(g_{i}(k)-g_{i}(k-1)\right) \leq R_{i}
\end{gathered}
$$

\footnotetext{
${ }^{2}$ Storage losses do have a cost implication on the overall dispatch, but those are implicitly handled by using conventional generation assets in meeting the power balance. Also, as we are considering real-time dispatch, reserve capacity holding charges are not considered since those are settled in the unit commitment.
} 
Dispatch for Storage $i \in \mathcal{I}_{s}$ at Step $k$ : Similarly, constraints (3.23)-(3.26) bound the energy storage devices by their state of charge and rated power output. The binary variable $u_{i}$ ensures that charging, $s_{i}^{\uparrow}(k)$, and discharging, $s_{i}^{\downarrow}(k)$, remain mutually exclusive.

$$
\begin{gathered}
s_{i}(k)=s_{i}^{\uparrow}(k)+s_{i}^{\downarrow}(k) \\
u_{i}(k) E_{i}^{\min } \leq s_{i}^{\downarrow}(k) \leq u_{i}(k) s_{i}(k-1) \\
\left(1-u_{i}(k)\right) s_{i}(k-1) \leq s_{i}^{\uparrow}(k) \leq\left(1-u_{i}(k)\right) E_{i}^{\max } \\
-P_{i} \leq \frac{60}{T}\left(s_{i}(k)-s_{i}(k-1)\right) \leq P_{i}
\end{gathered}
$$

In (3.26), we notice that charging and discharging efficiencies are not present because $s_{i}(k)$ and $s_{i}(k-1)$ represent control set points. The effects of the storage inefficiencies are taken into account in the power balance (3.20).

Flexible Energy Requirement Envelope for $m=1, \ldots, 12$ : Equations (3.27) and (3.28) generate the flexible energy requirement envelope by integrating the flexible power requirement envelope (second approach in Section 3.2.3)

$$
\begin{aligned}
W^{\uparrow}(m) & =\frac{T}{60} \sum_{j=1}^{m} \min \left(1.63 \sigma_{P}(j), 50-\ell(k)\right) \\
W^{\downarrow}(m) & =\frac{T}{60} \sum_{j=1}^{m} \max \left(1.63 \sigma_{P}(j), \ell(k)-10\right)
\end{aligned}
$$

We note that $W^{\uparrow}(m)$ and $W^{\downarrow}(m)$ are not optimization variables. They are parameters used next to enforce flexible energy envelope enclosure.

Flexible Power Envelopes for Generators $i \in \mathcal{I}_{g}$ and $m=1, \ldots, 12$ : Constraints (3.29)(3.34) determine for each generator two flexible power trajectories, one upward $x_{i}^{\uparrow}$ and one downward $x_{i}^{\downarrow}$, bounded by the generators' capacities and ramp rates. Here, (3.29) and (3.32) set the initial condition of the envelope to the step $k$ dispatch levels, while (3.31) and (3.34) bound the envelope trajectories to minimum and maximum generator capacity at the end of the receding horizon. On the other hand, (3.30) and (3.33) enforce maximum 
ramping limitations over the duration of the receding horizon.

$$
\begin{gathered}
x_{i}^{\downarrow}(0)=g_{i}(k) \\
\frac{1}{T}\left(x_{i}^{\downarrow}(m)-x_{i}^{\downarrow}(m-1)\right) \leq-R_{i} \\
x_{i}^{\downarrow}(12) \geq g_{i}^{\min } \\
x_{i}^{\uparrow}(0)=g_{i}(k) \\
\frac{1}{T}\left(x_{i}^{\uparrow}(m)-x_{i}^{\uparrow}(m-1)\right) \leq R_{i} \\
x_{i}^{\uparrow}(12) \leq g_{i}^{\max }
\end{gathered}
$$

Flexible Energy Envelopes for Storage $i \in \mathcal{I}_{s}$ and $m=1, \ldots, 12$ : In a way similar to the flexible power envelopes for generation, constraints (3.35)-(3.40) induce two flexible energy trajectories, one upward $z_{i}^{\uparrow}$ and one downward $z_{i}^{\downarrow}$, bounded by the assets' storage energy capacity and rated power. Equalities (3.35) and (3.38) enforce the initial condition of the energy flexibility envelope to the current dispatch step state of charge, while (3.37) and (3.40) guarantee that, at the end of the receding horizon, the flexible energy envelope is bounded by the minimum and maximum state of charge levels. In between, changes in the storage state of charge are limited by the rated power of storage assets, (3.36) and (3.39).

$$
\begin{aligned}
z_{i}^{\downarrow}(0) & =s_{i}(k) \\
\frac{60}{T}\left(z_{i}^{\downarrow}(m)\right. & \left.-z_{i}^{\downarrow}(m-1)\right) \leq-P_{i} \\
z_{i}^{\downarrow}(12) & \geq E_{i}^{\min } \\
z_{i}^{\uparrow}(0) & =s_{i}(k) \\
\frac{60}{T}\left(z_{i}^{\uparrow}(m)\right. & \left.-z_{i}^{\uparrow}(m-1)\right) \leq P_{i} \\
z_{i}^{\uparrow}(12) & \leq E_{i}^{\max }
\end{aligned}
$$

Energy Envelopes Enclosure for $m=1, \ldots, 12$ : Constraints (3.41) and (3.42) serve to enclose the flexible energy requirement envelope defined in (3.27) and (3.28) using both storage and conventional generation. These constraints are only to be used with the second energy storage strategy, which plans storage deployment anticipatorily. In the case where 
storage use is planned myopically, the storage terms are omitted and only generation is used to ensure envelope enclosure.

$$
\begin{aligned}
& \frac{T}{60} \sum_{j=1}^{m} \sum_{i \in \mathcal{I}_{g}}\left(x_{i}^{\uparrow}(j)-g_{i}(k)\right)+\sum_{i \in \mathcal{I}_{s}} \mu^{d}\left(s_{i}(k)-z_{i}^{\downarrow}(m)\right)=W^{\uparrow}(m) \\
& \frac{T}{60} \sum_{j=1}^{m} \sum_{i \in \mathcal{I}_{g}}\left(g_{i}(k)-x_{i}^{\downarrow}(j)\right)+\sum_{i \in \mathcal{I}_{s}} \frac{1}{\mu^{c}}\left(z_{i}^{\uparrow}(m)-s_{i}(k)\right)=W^{\downarrow}(m)
\end{aligned}
$$

Finally, the base strategy (for benchmarking the other two) does not use any storage. It uses the conventional generators only, to enclose the flexible energy requirement envelope. Hence, in this case all storage terms are out while all storage-related constraints are removed as well.

\subsubsection{Results and Discussion}

To compare quantitatively the three dispatch strategies, the energy dispatched (MWh) for a single realization $j, \mathrm{EG}_{j}$, contributed by the conventional resources is

$$
\mathrm{EG}_{j}=\frac{1}{12} \sum_{i=1}^{5} \sum_{k=1}^{12} g_{i, j}(k)
$$

whereas the cost $(\$)$ of dispatch for the same single realization $j, \mathrm{C}_{j}$, incurred by the conventional resources is

$$
\mathrm{C}_{j}=\frac{1}{12} \sum_{i=1}^{5} \sum_{k=1}^{12} c_{i} g_{i, j}(k)
$$

The expected energy (MWh) contribution by conventional resources for $J$ realizations is

$$
\mathrm{EG}_{J}=\frac{1}{J} \sum_{j=1}^{J} \mathrm{EG}_{j}
$$


whereas the total expected cost (\$) of operating the conventional resources for $J$ realizations is

$$
\mathrm{EC}_{J}=\frac{1}{J} \sum_{j=1}^{J} \mathrm{C}_{j}
$$

where $g_{i, j}(k)$ is the power dispatch of conventional resource $i$ at the $k^{\text {th }}$ dispatch step for the $j^{\text {th }}$ economic dispatch simulation.

Fig. 3.15 plots the histograms of (3.44) for the three dispatch strategies. Deploying storage myopically shifts and tightens the cost histogram over the basecase, implying reduced energy contributions and dispatch costs from the conventional resources. On the other hand, deploying storage anticipatorily via the energy envelopes approach further shifts and tightens the histogram.

Table 3.3 shows the values associated to (3.45) and (3.46). The storage dispatch strategy exploiting the flexible energy envelopes improves substantially over its myopic counterpart. Moreover, it is important to see how the capabilities of the storage assets are leveraged by considering their potential deployment moving forward. We note here that no forecast beyond the net load persistence forecast was needed here.

Table 3.3 Expected Energy Dispatched and Expected Costs of Conventional Resources

\begin{tabular}{lccccc}
\hline & No storage & Myopic & $\Delta$ & Envelopes & $\Delta$ \\
\hline EEG $(\mathrm{MWh})$ & 29.6 & 19.6 & $-34 \%$ & 6.7 & $-77 \%$ \\
\hline EC $\left(\$ \times 10^{3}\right)$ & 15.8 & 8.63 & $-45 \%$ & 2.50 & $-84 \%$ \\
\hline
\end{tabular}

Fig. 3.16 plots the histogram of the small (25 MWh) energy storage's intra-hourly state of charge, sampled every 5 minutes. It appears that optimal operations via the envelopes approach attempts to push the storage asset's state of charge as low as possible. This involves two simultaneous actions. One action is the discharge of the energy stored to deliver (dispatch) energy here and now, whereas the other action is the prepositioning of the state of charge at a lower level to provide potential fast-acting downward flexibility going forward.

On the other hand, Fig. 3.17 plots the histogram of the large ( $75 \mathrm{MWh}$ ) energy storage's 

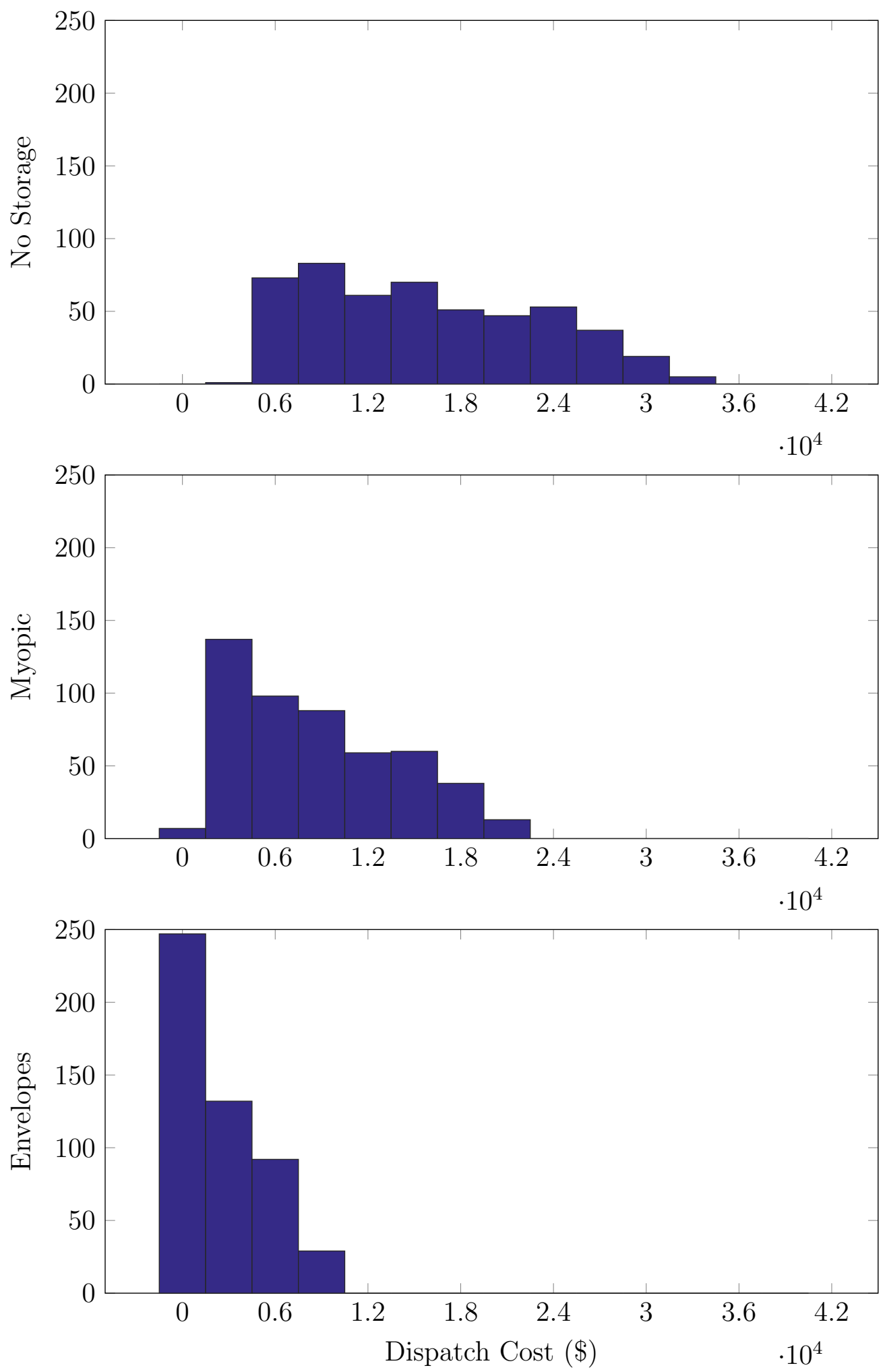

Fig. 3.15 Histograms of generators' total dispatch cost under the three economic dispatch strategies. 

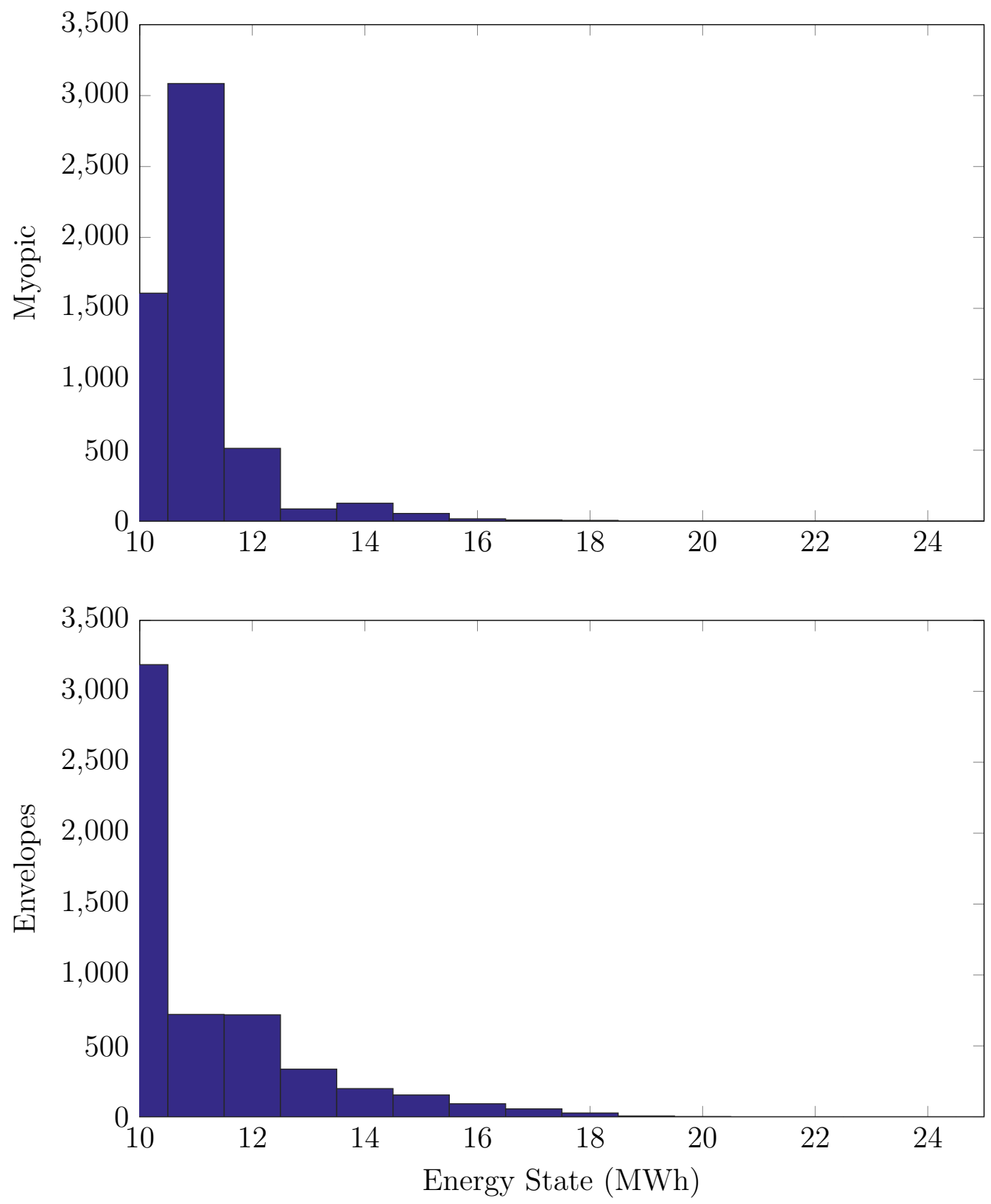

Fig. 3.16 Histograms of the 25 MWh storage's state of charge. 
intra-hourly state of charge, sampled every 5 minutes. The large storage asset has more capacity (in MWh) but a lower rate of charge/discharge (in MW), when compared to the small $25 \mathrm{MWh}$ asset. Here operations via the envelopes approach attempts to shift the expected state of charge to a lower level, as well as flatten its distribution. As expected, this shows that the $75 \mathrm{MWh}$ storage asset acts more as an energy buffer, by spending roughly equal time over a wide range of states of charge as it caters to the slower net load variations. In contrast, the $25 \mathrm{MWh}$ energy storage asset returns frequently to its lowest state of charge, thus catering to the faster net load variations.

\subsection{Summary}

In this chapter, we looked at flexibility as deployable energy, rather than the traditional view as deployable power. This prompted us to re-model the flexibility envelopes of Chapter 2 in terms of potential energy deployment. The new model facilitates the planning of energyconstrained resources (like energy storage systems and some forms of demand response). It allows for their energy levels could be prepositioned ahead of time to better optimize their potential deployment over an upcoming operational horizon. A proof of concept was performed through a receding-horizon economic dispatch problem to show how the an anticipatory storage strategy exploiting the proposed flexible energy envelope model is able to perform significantly better than with a myopic storage use strategy. 

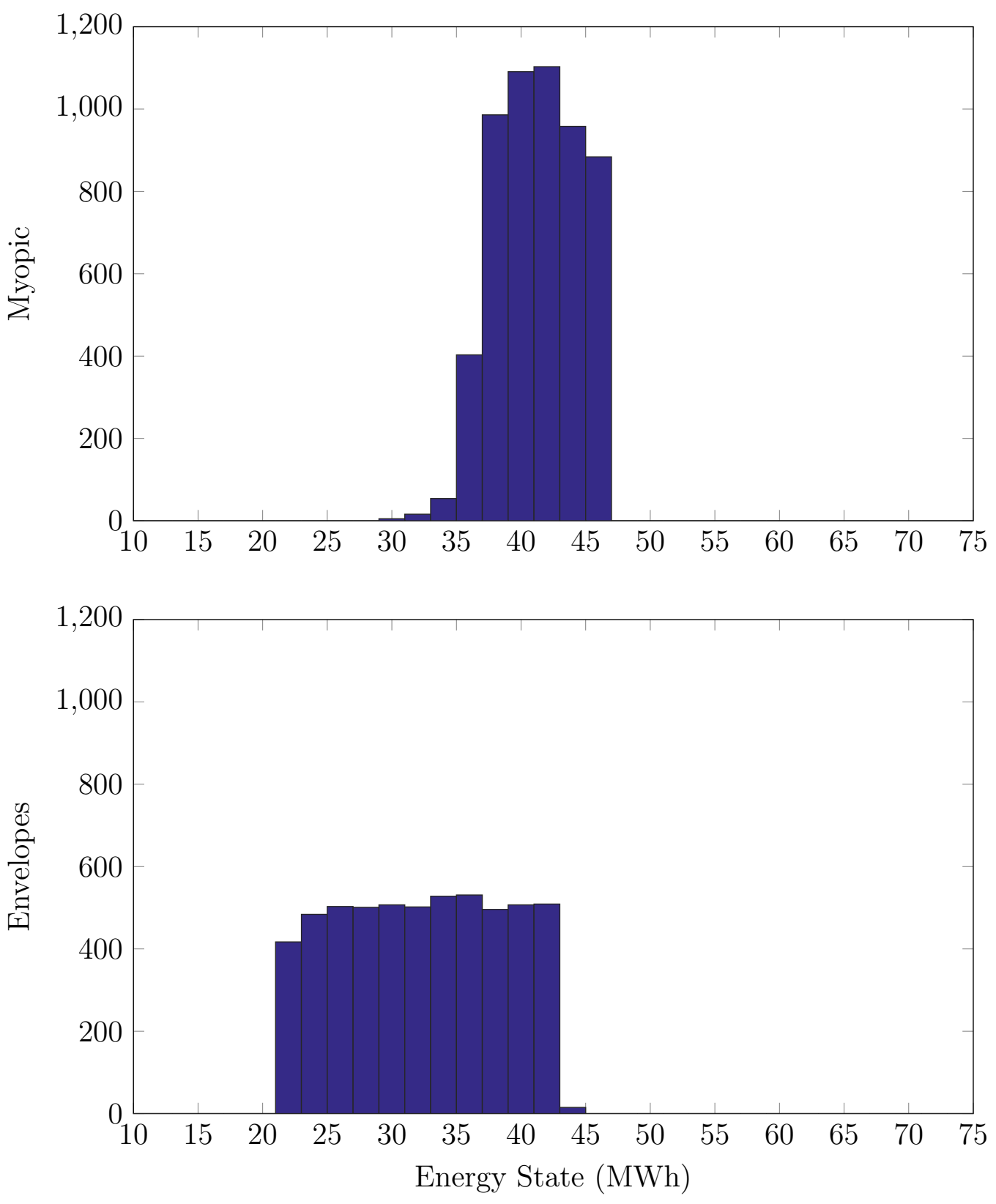

Fig. 3.17 Histogram of the 75 MWh storage's state of charge. 


\section{Chapter 4}

\section{Probabilistic Planning with Flexibility Envelopes}

With the emphasis on renewable energy integration, stochastic methods have begun to emerge as a viable alternative to traditional planning under uncertainty. The value of stochastic planning lies in its effectiveness at reducing operational costs, while allowing higher penetration levels of renewable sources, and without much sacrifice to system security. This is under the premise that the decision maker may not need to fully hedge against more extreme and costlier events that exhibit lower probabilities of occurrence. On the other hand, stochastic methods do suffer from the "curse of dimensionality," which renders them computationally intractable for practical application. Here, we propose a new stochastic approach based on the flexibility envelopes concept that was proposed in Chapter 2. It circumvents the curse of dimensionality by using probability-weighted envelopes to enclose the evolution of the net load uncertainty over the planning horizon. The new approach is illustrated by a receding-horizon economic dispatch example, to compare its effectiveness to the earlier deterministic version that used a single envelope.

\subsection{Introduction}

The power system infrastructure is undergoing a transitional phase, whereby the integration of renewable energy sources (RES) and the active participation of electricity consumers are reshaping the characteristics of the net load (i.e. load less RES output). As a result, the industry is facing a new challenge of increased variability and uncertainty in the power 
balance, beyond what legacy operation and planning practices were designed to handle. To answer this challenge, stochastic-based planning has recently emerged to better handle this inherent variability and uncertainty in the net load, while the emerging concept of power system flexibility is expected to phase out the traditional operating reserve definitions, in due time [5]. Stochastic planning and power system flexibility go hand-in-hand, given that stochastic methods compute operating reserve implicitly, without requiring explicit reserve definitions in the traditional sense. This provides more unity across power systems by alleviating the lack of consensus on the explicit definitions of reserve types [13]. That is, power system flexibility requirements are best characterized implicitly by the net load variability and uncertainty dynamics, rather than explicitly by categorical reserve typessuch as primary, secondary, and tertiary reserves.

In stochastic planning [9], variability and uncertainty are modeled directly in the planning problem, in the form of probability-weighted scenarios, to which the optimal dispatch/scheduling solution must be probabilistically robust, not fully. This allows the system operator to implicitly reduce reserve requirements arising from the scenarios having lower probabilities of occurrence but higher reserve costs. In turn, this can have a substantial impact on reducing the operational and planning costs, for a small tolerable reduction in system security. Here, the scenarios' dynamics implicitly capture the net load variability and uncertainty characteristics, and there is no need to explicitly handle the traditional reserve types within the planning model.

However, one serious drawback to scenario-based stochastic planning is known as the "curse of dimensionality." It refers to the exponentially increasing computational requirements resulting from the exponentially growing possible outcomes of the evolution of the net load variability and uncertainty over the planning horizon. Consequently, the applicability of stochastic planning has been very limited at the inter-hourly scale (i.e. hourly unit commitment and tertiary reserve planning), whereas there hasn't been any work done at the intra-hourly scale pertaining to intra-hourly flexibility - involving primary and secondary reserve planning. All in all, stochastic optimization formulations are yet to emerge as viable practical industry solutions for operational planning.

On the other hand, scenario reduction techniques [23] can be used to partially circumvent the curse of dimensionality. However, this adds another layer of computational and modeling complexity to the planning problem, and the accuracy of the approximation degrades with the degree of scenario reduction, especially under high variability and 
uncertainty. This is even more so on the intra-hourly timescale, where the net load may become significantly volatile. Therefore, whereas scenario-based stochastic planning may be far from being computationally tractable for intra-hourly flexibility planning, an alternative stochastic approach that is simplistic and computationally viable is still desired. To that end, we propose the probabilistic envelopes approach to power system planning under uncertainty.

In Chapter 2, we proposed the concept of flexibility envelopes for power system operational planning. We used envelopes to capture the intra-hourly flexibility deployment characteristics of resources and the intra-hourly flexibility requirement characteristics arising from the net load variability and uncertainty. Such envelope enclosed statistically a certain percentile of the plausible net load realizations (i.e. stochastic scenarios). We asserted flexibility adequacy is achieved when the aggregate flexibility envelope of resources encloses the flexibility requirement envelope over the operational horizon.

The underlying strategy behind the flexibility envelopes approach is essentially a minmax strategy [60]. Whereas scenario-based stochastic planning is robust with respect to a large number of probability-weighted scenarios, a min-max strategy needs only to be robust with respect to the worst-case scenario(s) (i.e. the flexibility requirement envelope) to guarantee robustness with respect to all enclosed scenarios within the envelope. This is clearly advantageous, computational-wise, over scenarios-based stochastic planning. The downside of min-max planning is that it results in strict reserve requirements - pertaining to the worst-case scenario(s) - at the expense of higher expected operating costs. This, however, can be controlled by determining the percentile of scenarios enclosed by the flexibility requirement envelope, as elaborated in Chapter 2.

In this Chapter, we extend the flexibility envelopes concept, introduced in Chapter 2, by quantifying several flexibility requirement envelopes enclosing an increasing order of percentiles of the net load uncertainty. During planning, we require the aggregate response of resources to probabilistically enclose those envelopes, by taking into account their probability weights. The advantage of such an approach lies in the absence of coupled exponentially growing scenarios, thus alleviating the curse of dimensionality-because computational requirements grow linearly with the number of envelopes and the length of the planning horizon. Moreover, the envelopes and their probabilities are easy to calculate and incorporate in a linear program formulation.

Alternatively one may think of the proposed probabilistic envelopes approach as a sce- 
nario reduction process, because each envelope encloses a corresponding percentile of scenarios. In other words, a certain percentile of scenarios can be aggregated into an envelope trajectory that represents the capacity and ramping limit-behavior of the scenarios enclosed within. The advantage over available scenario-reduction techniques is that the envelopes are easy to calculate statistically from the empirical net load data. Hence, there is no need for constructing a scenario tree, generating Monte Carlo scenarios, or applying complex scenario reduction algorithms.

\subsection{The Flexibility Requirement Envelope}

Let persistence forecast be the most recent measurement of the net load at present time $k$, which is used as a reference level for computing flexibility requirements over the planning horizon. In other words, flexibility deployment is required when the net load deviates from the persistence forecast over the operational horizon. Let $\Delta P(\tau ; k)$ define a step change in the net load power time series $\ell(k)$, realized $\tau$ units of time later, as seen from the present time $k$ :

$$
\Delta P(\tau ; k)=\ell(k+\tau)-\ell(k)
$$

Given an empirical net load time series $\ell(k)$, the sample realizations $\Delta P(\tau ; k)$ can be obtained by fixing $\tau$ and sweeping through $k$ in the time series $\ell(k)$. Consequently, the flexibility requirement envelope at $\tau$ quantifies the $p^{\text {th }}$ percentile of $\Delta P(\tau ; k)$ both upward and downward, where $\tau$ varies from zero to $H$ (the length of the envelope's horizon). Here, we use the Laplace distribution to fit the $\Delta P(\tau ; k)$ realizations, as this has been the preferred distribution for wind power variability in several wind power integration studies $[51,61]$ :

$$
f(\Delta P(\tau ; k) \mid \tau)=\frac{1}{2 \beta(\tau)} \exp \left(-\frac{|\Delta P(\tau ; k)-\mu(\tau)|}{\beta(\tau)}\right)
$$

where $f$ is the symmetric Laplace $\mathrm{PDF}, \mu(\tau)$ is the mean, and $\beta(\tau)>0$ is the scale parameter. Both $\mu(\tau)$ and $\beta(\tau)$ change with $\tau$, while we assume the Laplace structure does 
not change. The standard deviation $\sigma(\tau)$ is a function of $\beta(\tau)$ :

$$
\sigma(\tau)=\beta(\tau) \sqrt{2}, \quad \tau=0, \ldots, H
$$

Fig. 4.1 shows the Laplace PDF discretized uniformly by integer increments of the standard deviation, where $\mu=0$ and $\beta=1$. As shown in the figure, for the Laplace distribution, it suffices to consider up to five increments of the standard deviation, to cover most of the probability. The $p_{q}^{t h}$ percentile corresponding to the $q^{\text {th }}$ increment of $\sigma$ is

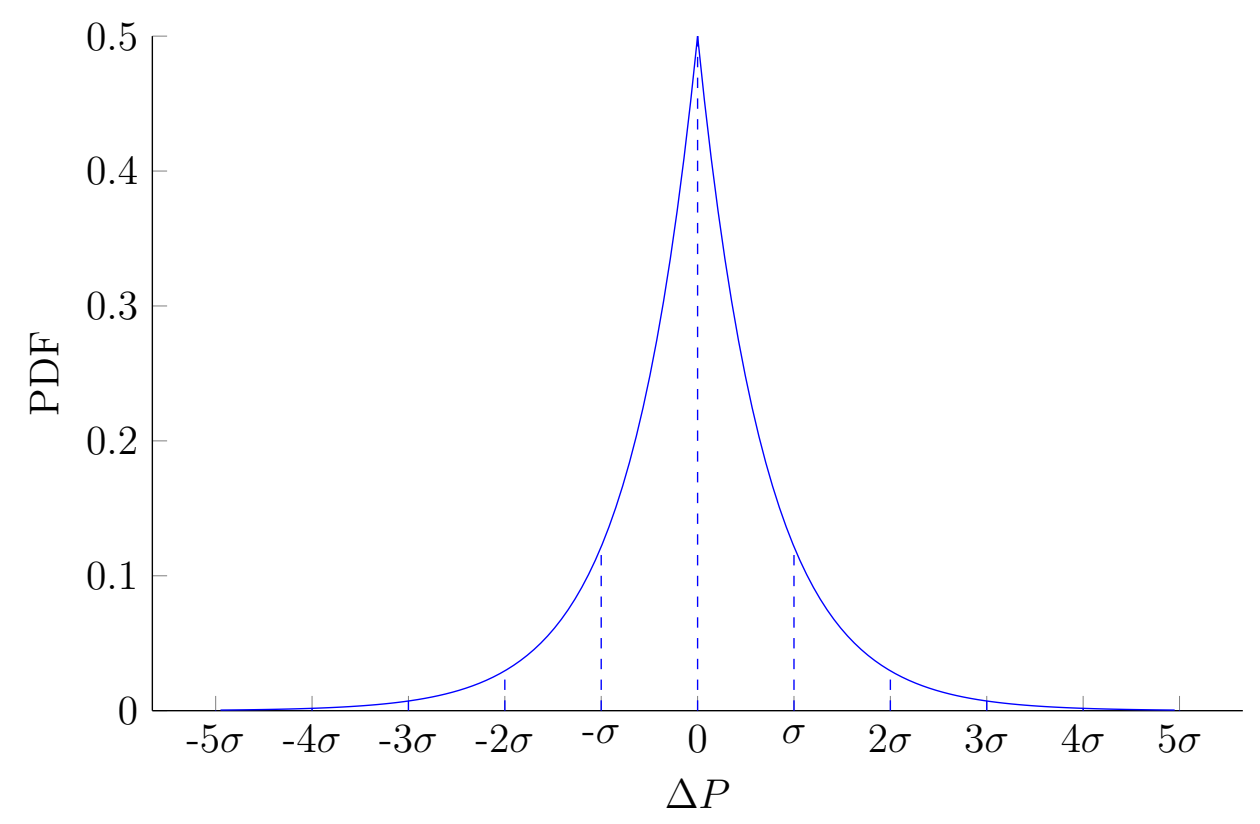

Fig. 4.1 Laplace PDF given $\mu=0$ and $b=1$. The dashed lines discretize the PDF uniformly by integer increments of $\sigma=\beta \sqrt{2}$

$$
\begin{aligned}
p_{q} & =\mathrm{P}(\mu-q \sigma \leq \Delta P \leq \mu+q \sigma) \\
& =(\operatorname{CDF}(\mu+q \sigma)-\operatorname{CDF}(\mu-q \sigma))
\end{aligned}
$$

where $q=1, \ldots, 5$ and $\mathrm{CDF}$ is the cumulative distribution function of (4.2). Table 4.1 shows the actual $p_{q}^{\text {th }}$ percentiles of the symmetric Laplace distribution of Fig. 4.1, which will be used later for probabilistic planning with the envelopes. Moreover, a non-uniform discretization of $q$, based on $20 \%$ increments of the enclosed percentile $p_{q}$, is shown in 
Table 4.1 Percentiles of the symmetric Laplace distribution for integer increments of the standard deviation

\begin{tabular}{cccccc}
\hline$q$ & 1 & 2 & 3 & 4 & 5 \\
\hline$p_{q}$ & 0.757 & 0.941 & 0.986 & 0.997 & 0.999 \\
\hline
\end{tabular}

Table 4.2, which will also be used for probabilistic planning, to compare its performance relative to the aforementioned uniform discretization. The upward and downward envelope

Table 4.2 Percentiles of the symmetric Laplace distribution for $20 \%$ increments of the percentiles

\begin{tabular}{cccccc}
\hline$q$ & 0.158 & 0.361 & 0.648 & 1.138 & 4.885 \\
\hline$p_{q}$ & 0.2 & 0.4 & 0.6 & 0.8 & 0.999 \\
\hline
\end{tabular}

trajectories, $\left\{e_{q}^{\uparrow}(\tau), e_{q}^{\downarrow}(\tau)\right\}$, of a single envelope based on the $q^{\text {th }}$ increment of $\sigma$ become

$$
\begin{aligned}
e_{q}^{\uparrow}(\tau) & =q \sigma(\tau)=q \beta(\tau) \sqrt{2} ; & & \tau=0, \ldots, H \\
e_{q}^{\downarrow}(\tau) & =-q \sigma(\tau)=-q \beta(\tau) \sqrt{2} ; & & \tau=0, \ldots, H
\end{aligned}
$$

Therefore, $\sigma(\tau)$ or $\beta(\tau)$ must be fitted empirically at every $\tau$ to obtain the envelopes' trajectories. As an example, consider the same net load data obtained for the BPA power system [59] used throughout the dissertation. The sampling period is 5 minutes, and $\tau$ is set to range from zero to 60 minutes in increments of 5 minutes. Fitting the Laplace distribution at every $\tau$, Table 4.3 shows the standard deviation $\sigma(\tau)$ as a function of $\tau$. Fig. 4.2 plots the flexibility requirement envelopes for $q=1, \ldots, 5$, over a one-hour planning horizon.

Finally, each $q$ value corresponds to a single flexibility requirement envelope which captures the intra-hourly characteristics of the net load in terms of capacity and ramping requirements, for the $p_{q}^{\text {th }}$ percentile of realizations (scenarios) it encloses.

\subsection{The Flexibility Envelope of Power System Resources}

In line with the previous section, a planned deviation from the present (scheduled) power level of a power system resource is what constitutes flexibility deployment. Let $y(k)$ repre- 
Table 4.3 The standard deviation of the BPA data as a function of $\tau$

\begin{tabular}{cc}
\hline$\tau$ (minutes) & $\sigma(\tau)(\mathrm{MW})$ \\
\hline 5.0 & 59.2 \\
10.0 & 90.1 \\
15.0 & 119.9 \\
20.0 & 148.7 \\
25.0 & 177.6 \\
30.0 & 205.6 \\
35.0 & 233.6 \\
40.0 & 261.0 \\
45.0 & 287.5 \\
50.0 & 313.4 \\
55.0 & 339.8 \\
60.0 & 366.0
\end{tabular}

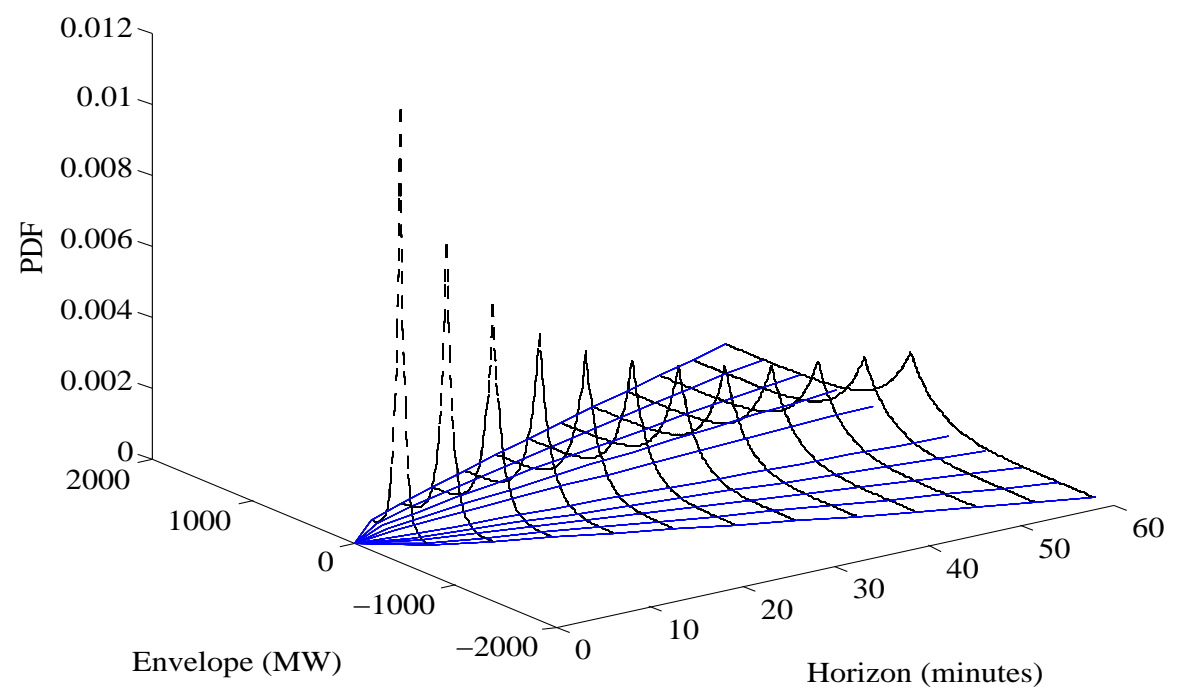

Fig. 4.2 The flexibility requirement envelopes of the BPA data for one hour duration, computed as integer increments of the standard deviation-Fig. 4.1 
sent the power output level at present time $k$. The output power can undergo step changes as flexibility is deployed over forward time $\tau$

$$
\Delta P(\tau ; k)=y(k+\tau)-y(k)
$$

The reader may refer to Chapter 2 and Chapter 3 for illustrative examples on calculating the flexibility envelope of a power system resource. To re-emphasize, a flexibility envelope captures the intra-hourly characteristics of a power system resource, in term of capacity and ramping requirements. On the other hand, the aggregate flexibility envelope captures the intra-hourly characteristics of all resources combined, depending on how the individual envelopes are prepositioned with respect to their scheduled output levels.

\subsection{Probabilistic Planning with Envelopes}

We asserted in Chapter 2 that the aggregate flexibility envelope by all resources must enclose the flexibility requirement envelope, to guarantee adequate system flexibility in terms of capacity and ramping requirements. Here, we extend the concept to probabilistic envelope enclosure, by enclosing the $p_{q}^{t h}$ percentile flexibility requirement envelopes, where

$q$ is discretized according to Table 4.1 or Table 4.2. Next, we present this novel planning approach via a simple example.

\subsubsection{Problem Setup}

Receding Horizon Operation: Fig. 4.3 schematically illustrates flexibility-constrained recedinghorizon planning for a single decision-making epoch. The epoch may represent a single unit commitment hour, such that the envelopes are computed once at the beginning of each hour. Here, however, it represents a single economic dispatch period, such that the envelopes are projected onto the future and are updated in a receding-horizon fashion at every dispatch step $k$. As illustrated by Fig. 4.3, the power system is running during the $h^{\text {th }}$ hour. The decision maker must re-dispatch the committed resources to match the new forecast load $\ell(h+k)$, by ramping these resources from their previous output levels at time $h+k-1$, subject to ramping constraints. Concurrently, the decision maker must preposition these resources at time $h+k$, such that their projected aggregate flexibility envelope encloses the probability-weighted flexibility requirement envelopes over the upcoming operational hori- 
zon $h+k+m$, where $\tau=m T$ is the envelopes' receding horizon and $T$ is the discretization period. There are 12 economic dispatch periods within the single unit commitment hour, such that the discretization interval is $T=5$ minutes and $k=1, \ldots, 12$. The projected horizon of the envelopes is set to one hour, such that $m=1, \ldots, M$, where $M=12$.

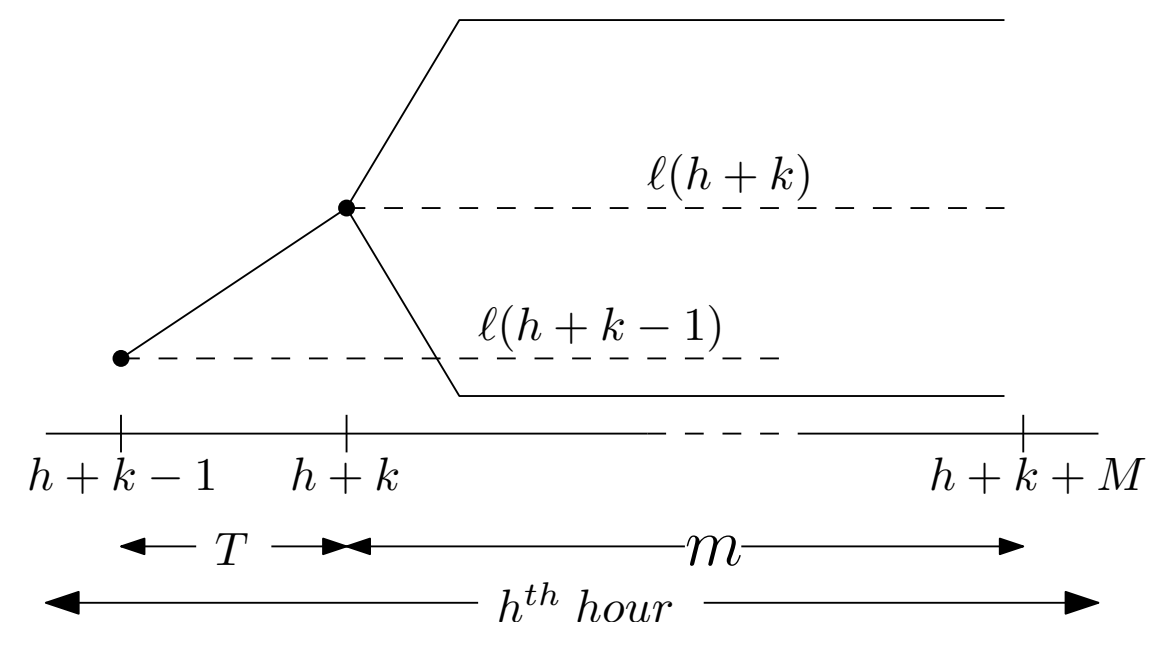

Fig. 4.3 Schematic illustration of a single decision making epoch

Net Load Model: A series of twelve 5-minute step changes, $\{w(k)\}_{k=1}^{12}$, is generated randomly from a symmetric Laplace distribution that is characterized by $\beta=0.05 \mathrm{MW} /$ minute. The step changes are assumed to be independent and identically distributed (i.i.d.), $\sqrt{2} \beta$ is the standard deviation, and $q \sqrt{2} \beta$ is the $p_{q}^{\text {th }}$ percentile according to Table 4.1 or Table 4.2. The initial net load $\ell(0)$ forecast is set to $30 \mathrm{MW}$. The net load forecasts for the economic dispatch periods are generated by subsequently adding the random step changes $\{w(k)\}_{k=1}^{12}$ to the initial net load $\ell(0)$. Fig. 4.4 plots sample realizations of the net load random process bounded by a flexibility requirement envelopes covering the percentiles in Table 4.1.

Therefore, the power deviation (step change) as a function of $\tau=m T$ becomes

$$
\Delta P(m)=\sum_{i=1}^{m} w(i) ; \quad m=1, \ldots, 12
$$

The standard deviation $\sigma_{P}(m)$ is computed by taking the variance of (4.8) and noting the 


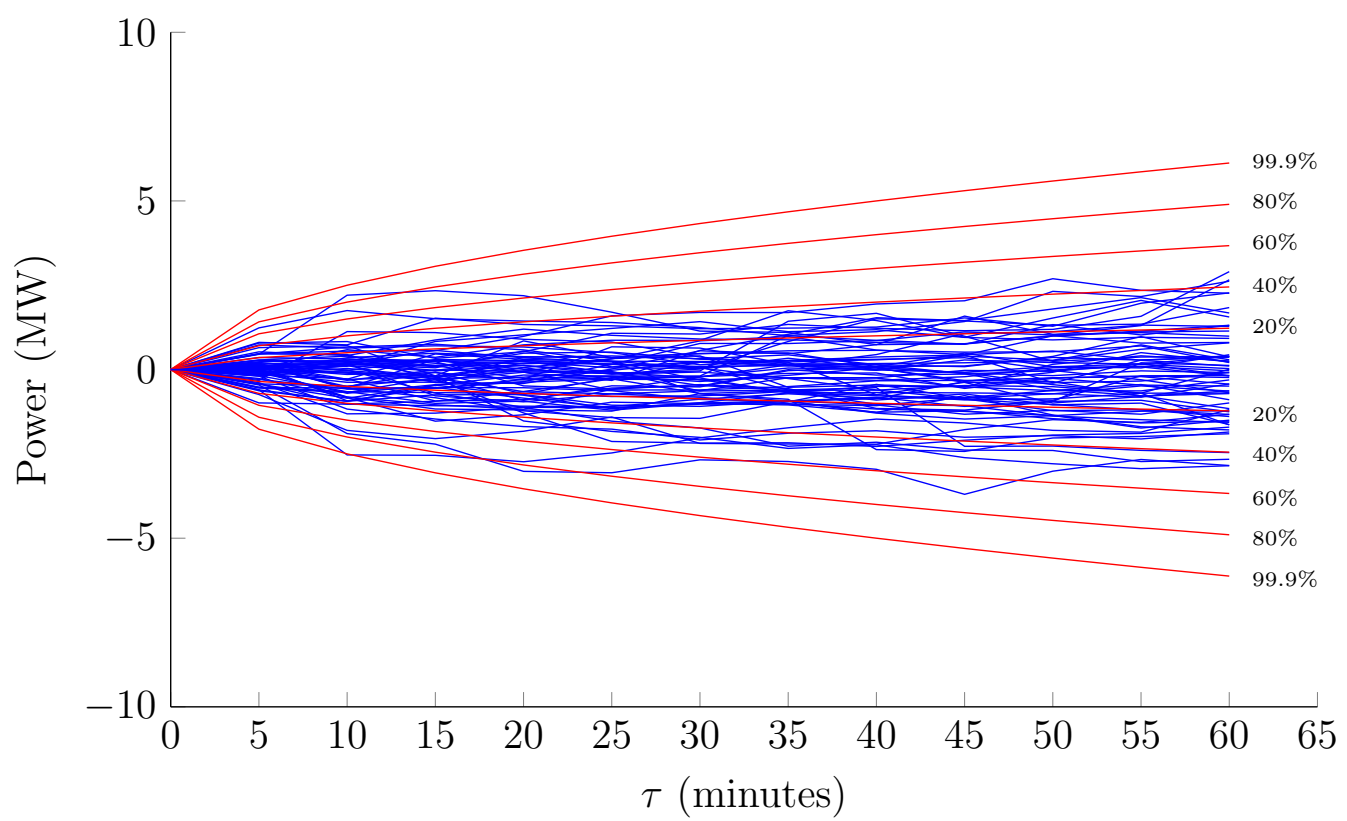

Fig. 4.4 Fifty sample realizations of the net load random process (in blue) bounded by the flexibility requirement envelopes according to Table 4.1 (in red).

i.i.d. property ${ }^{1}$ of $\left\{w_{k}\right\}_{k=1}^{12}$, thus yielding

$$
\sigma_{P}(m)=\sqrt{m} \sqrt{2} \beta ; \quad m=1, \ldots, 12
$$

Here, the Laplace distribution is assumed to hold for all $\tau=m T$, as an approximation of the actual distributions. Multiplying (4.9) by a factor of $q$ yields the flexibility requirement envelope covering the $p_{q}$ confidence region. The Laplace PDF is discretized using 5 percentiles, uniformly according to Table 4.1, such that $q \in \mathcal{Q}$ where $\mathcal{Q}=\{1,2,3,4,5\}$, or non-uniformly according to Table 4.2 , such that $q \in \mathcal{Q}$ where $\mathcal{Q}=\{0.158,0.361,0.648,1.138,4.885\}$

Flexibility Resources: There is a set $\mathcal{I}_{g}$ of five identical conventional resources providing flexibility, which have incremental costs $\left\{b_{i}\right\}_{i \in \mathcal{I}_{g}}$. Table 4.4 shows the cost and flexibility characteristics of the five resources.

\footnotetext{
${ }^{1}$ We recall that the covariance of i.i.d. random variables is zero.
} 
Table 4.4 Resources' Capacity, Ramping, and Cost Characteristics

\begin{tabular}{ccccc}
\hline & $g^{\max }(\mathrm{MW})$ & $g^{\min }(\mathrm{MW})$ & $R(\mathrm{MW} /$ minute $)$ & $b(\$ / \mathrm{MW})$ \\
\hline 1 & 10 & 2 & 0.0154 & 20 \\
2 & 10 & 2 & 0.0154 & 40 \\
3 & 10 & 2 & 0.0154 & 60 \\
4 & 10 & 2 & 0.0154 & 80 \\
5 & 10 & 2 & 0.0154 & 100 \\
\hline
\end{tabular}

\subsubsection{Mathematical Program}

The following linear program is an extension of the linear program presented in Chapter 2 , to represent probabilistic envelope enclosure. The linear program in Chapter 2 used a single flexibility requirement envelope covering the majority of the probability, whereas here we use several probability-weighted flexibility requirements envelopes. For the sake of simplicity, $h+k$ is replaced by $k$, since $h$ remains fixed during the same hour of economic dispatch steps, and $h+k+m$ is replaced by $m$, since the hour $h$ and dispatch period $k$ remain fixed while projecting the envelopes over the receding horizon steps $m$.

Objective: We seek in (4.10) to minimize the cost of dispatching the resources $\mathcal{I}_{g}$ and slacks $S^{\uparrow \downarrow}$ at time $k$. Moreover, we seek to minimize the potential cost of flexibility deployment over the receding horizon $m=1, \ldots, M$, to enclose the probability-weighted flexibility requirement envelopes, while minimizing the cost of deploying the slack resources $e_{S}^{\uparrow \downarrow}$ over the horizon $m=1, \ldots, M$ to help enclose the probability-weighted flexibility requirement envelopes. The optimal cost must find a balance between these opposing sub-objectives.

$$
\begin{aligned}
& \min \sum_{i \in \mathcal{I}_{g}} b_{i} g_{i}(k)+c_{S}\left[S^{\uparrow}(k)-S^{\downarrow}(k)\right] \\
& +\sum_{q \in \mathcal{Q}} \sum_{m=1}^{M} b_{i} \frac{p(q)-p(q-1)}{2}\left[x_{i}^{\uparrow}(m, q)-x_{i}^{\downarrow}(m, q)\right] \\
& +\sum_{q \in \mathcal{Q}} \sum_{m=1}^{M} c_{S} \frac{p(q)-p(q-1)}{2}\left[e_{S}^{\uparrow}(m, q)-e_{S}^{\downarrow}(m, q)\right]
\end{aligned}
$$

This is subject to

Dispatch constraints at $k$ : The combined resources and slack must balance the net load 
at dispatch time $k$, while respecting the capacity and ramping limitations of resources, as they ramp from their previous dispatch at time $k-1$. The slacks, which effectively consist of load shedding $S^{\uparrow}$ and generation curtailment $S^{\downarrow}$, are assumed to have infinite flexibility in terms of capacity and ramping.

$$
\begin{array}{ll}
\sum_{i \in \mathcal{I}_{g}} g_{i}(k)+S^{\uparrow}(k)+S^{\downarrow}(k)=\ell(k) & \\
g_{i}^{\min } \leq g_{i}(k) \leq g_{i}^{\max } ; & i \in \mathcal{I}_{g} \\
-R_{i} \leq g_{i}(k)-g_{i}(k-1) \leq R_{i} ; & i \in \mathcal{I}_{g} \\
S^{\uparrow}(k) \geq 0 & \\
S^{\downarrow}(k) \leq 0 &
\end{array}
$$

Resources Flexibility Envelopes for $q \in \mathcal{Q}, m=1, \ldots, M$ and $i \in \mathcal{I}_{g}$ : Each resource $i \in \mathcal{I}_{g}$ generates a pair of flexibility potential trajectories $x_{i}^{\uparrow \downarrow}(m, q)$ over the receding horizon $m=1, \ldots, M$, one pair for each $q \in \mathcal{Q}$, which are constrained by capacity and ramping limitations.

$$
\begin{gathered}
g_{i}^{\min } \leq x_{i}^{\downarrow}(m, q) \leq g_{i}(k) \\
g_{i}(k) \leq x_{i}^{\uparrow}(m, q) \leq g_{i}^{\max } \\
-m \cdot R_{i} \leq x_{i}^{\downarrow}(m, q)-g_{i}(k) \leq 0 \\
0 \leq x_{i}^{\uparrow}(m, q)-g_{i}(k) \leq m \cdot R_{i}
\end{gathered}
$$

Envelopes Matching for $q \in \mathcal{Q}$ and $m=1, \ldots, M$ : The above trajectories $x_{i}^{\uparrow \downarrow}(m, q)$ are used to enclose each of the $q \in \mathcal{Q}$ probability-weighted flexibility requirement envelopesthe right-hand sides of (4.20) and (4.21) - over the receding horizon $m=1, \ldots, M$. This enclosure is supplemented by the slack resources $e_{S}^{\uparrow \downarrow}(m, q)$ which have infinite flexibility like the dispatch slack resources $S^{\uparrow \downarrow}$. The evolution of the upper and lower half-segments of the probability-weighted flexibility requirement envelopes are described on the right-hand 
side of (4.20) and (4.21), respectively.

$$
\begin{gathered}
\sum_{i \in \mathcal{I}_{g}}\left(x_{i}^{\uparrow}(m, q)-g_{i}(k)\right)+e_{S}^{\uparrow}(m, q) \geq q \sqrt{m} \sqrt{2} \beta \\
\sum_{i \in \mathcal{I}_{g}}\left(g_{i}(k)-x_{i}^{\downarrow}(m, q)\right)-e_{S}^{\downarrow}(m, q) \geq q \sqrt{m} \sqrt{2} \beta \\
e_{S}^{\uparrow}(m, q) \geq 0 \\
e_{S}^{\downarrow}(m, q) \leq 0
\end{gathered}
$$

To re-emphasize, the problem (4.10)-(4.23) is solving the economic dispatch at time $k$ projecting the potential evolution of the net-load up to $M$ sub-hourly time steps ahead. Therefore, envelopes are always calculated based on the current state of the system when the economic dispatch is computed. So, if in between the current dispatch time $k$ and the previous sub-hourly dispatch step $k-1$ flexibility has been used, the envelopes of the resources which have deployed flexibility are updated based on their current operating point.

Ideally, the $\mathcal{I}_{g}$ resources should have enough flexibility to ramp from the previous time step $k-1$, while ensuring there is still enough flexibility to enclose the projected probabilityweighted flexibility requirement envelopes, moving forward. However, due to poor planning in the past or a lack of sufficient flexibility, the $\mathcal{I}_{g}$ resources may not be able to collectively position themselves at time step $k$ to balance the current net load or to ensure proper enclosure of the projected probability-weighted flexibility requirement envelopes moving forward. Consequently, the decision maker may have to deploy slack resources [in the form of load shedding $\left(S^{\uparrow}\right)$ and generation curtailment $\left(S^{\downarrow}\right)$ ] to avert dispatch infeasibility.

On the other hand, deploying the dispatch slacks $S^{\uparrow, \downarrow}$ is undesirable and thus should be priced much higher than the other resources - in this example, they are priced at $\$ 1000$ per MW per hour. They are to be used as a last resort when the problem becomes infeasible. To this end, the feasibility at $k$ can be improved by relaxing the enclosure of the probabilityweighted flexibility requirement envelopes - via the use of slack resource $e_{S}^{\uparrow}$ and $e_{S}^{\downarrow}$ in (4.20)(4.23). However, relaxing future flexibility requirements should also come at a cost, since it may lead to poor prepositioning of the $\mathcal{I}_{g}$ resources at the current time, which, in turn, may affect future flexibility availability. As such, the cost of envelope relaxation is reflected 
in the third line of $(4.10)$, which is also priced at $\$ 1000$ per MW per hour in this case.

Hence, the problem in (4.10)-(4.23) becomes that of balancing the current flexibility requirement (i.e. the ability to ramp from the previous dispatch time step to a feasible positioning at the current time step) with the future flexibility requirement (i.e. enclosing the projected probability-weighted flexibility requirement envelopes), while balancing the system's current net load. The trade-off depends on how the $\mathcal{I}_{g}$ resources, the slack resources and the envelope's slack variables are priced relative to one another in (4.10), given the relative probabilities of having to enforce specific envelopes.

\subsubsection{Results and Discussion}

We simulate seven economic dispatch policies, indicated in Table 4.5. For each of the first five policies, $\mathcal{Q}$ is set in the mathematical program to one of the values from Table 4.2 , while the terms $[p(q)-p(q-1)] / 2$ are removed from the objective function (4.10). This constitutes the deterministic envelope enclosure presented in Chapter 2.

Table 4.5 Simulated Economic Dispatch Policies

\begin{tabular}{cc}
\hline Policy & Envelope Enclosure \\
\hline 1 & single envelope enclosing the $20^{\text {th }}$ percentile \\
2 & single envelope enclosing the $40^{t h}$ percentile \\
3 & single envelope enclosing the $60^{t h}$ percentile \\
4 & single envelope enclosing the $80^{t h}$ percentile \\
5 & single envelope enclosing the $99.9^{t h}$ percentile \\
6 & non-uniform probabilistic according to Table 4.2 \\
7 & uniform probabilistic according to Table 4.1 \\
\hline
\end{tabular}

There are $J=1500$ one-hour long economic dispatch runs carried out for each dispatch policy, by generating 1500 net load realizations as described in Section 4.4.1. This is done to collect sufficient empirical data, to benchmark the three dispatch policies.

Performance Metrics: To assess the quality of the dispatch decisions made through the various policies, we calculate the energy served for each realization $j\left(\mathrm{ES}_{i, j}\right)$ and the expected energy served $\left(\mathrm{EES}_{i}\right)$ by each resource $i$. In addition, we compute the energy not served $\left(\mathrm{ENS}_{j}\right)$ and the energy curtailed $\left(\mathrm{EC}_{j}\right)$ for each realization $j$, from which we obtain the expected energy not served (EENS) and the expected energy curtailed (EEC) for all $J$ 
realizations of the net load random process.

$$
\begin{array}{rlrl}
\mathrm{ES}_{i, j} & =T \sum_{k=1}^{K} g_{i, j}(k) ; & i=1, \ldots, I ; j=1, \ldots, J \\
\mathrm{EES}_{i, J} & =\frac{1}{J} \sum_{j=1}^{J} \mathrm{ES}_{i, j} ; & & i=1, \ldots, I \\
\mathrm{ENS}_{j} & =T \sum_{k=1}^{K} S_{j}^{\uparrow}(k) ; & & \\
\mathrm{EENS}_{J} & =\frac{1}{J} \sum_{j=1}^{J} \mathrm{ENS}_{j} & j=1, \ldots, J \\
\mathrm{EC}_{j} & =T \sum_{k=1}^{K}-S_{j}^{\downarrow}(k) ; & \\
\mathrm{EEC}_{J}=\frac{1}{J} \sum_{j=1}^{J} \mathrm{EC}_{j} &
\end{array}
$$

where $g_{i, j}(k), S_{i, j}^{\uparrow}(k)$ and $S_{i, j}^{\downarrow}(k)$ correspond to the realizations of the economic dispatch decisions at time step $k$ for random net load realizations $j=1, \ldots, J$.

Because of the power balance imposed by (4.11), the sum of the expected values (4.25)(4.29) should converge to the expected net load energy (ENLE) requirement

$$
\operatorname{ENLE}_{J}=\frac{T}{J} \sum_{j=1}^{J} \sum_{k=1}^{K} \ell_{j}(k)
$$

which should be $30 \mathrm{MWh}$, given the initial net load forecast was set to $30 \mathrm{MW}$, and the Laplace-distributed net load variability model in Section 4.4.1 is symmetric about zero. The $J=1500$ simulations were sufficient, to achieve satisfactory convergence of the empirical expectations defined above.

Fig. 4.5 plots the expected energy served (EES) by the $\mathcal{I}_{g}$ resources, to meet the ENLE requirement. As shown, dispatch policies 2-4 performs the worst, in the sense that they do not fully meet the ENLE requirement of $30 \mathrm{MWh}$ - the remainder is covered by the slack resources. Moreover, they poorly utilize the second cheapest resource, when compared to the more expensive ones. Policies 1 (enclosing 20\%) and 5 (enclosing 99.9\%) perform similarly 
in terms of utilizing the $\mathcal{I}_{g}$ resources, but still do not fully meet the ENLE requirement, as well. On the other hand, the probabilistic policies 6 and 7 perform the best in utilizing the $\mathcal{I}_{g}$ resources to fully meet the ENLE requirement.

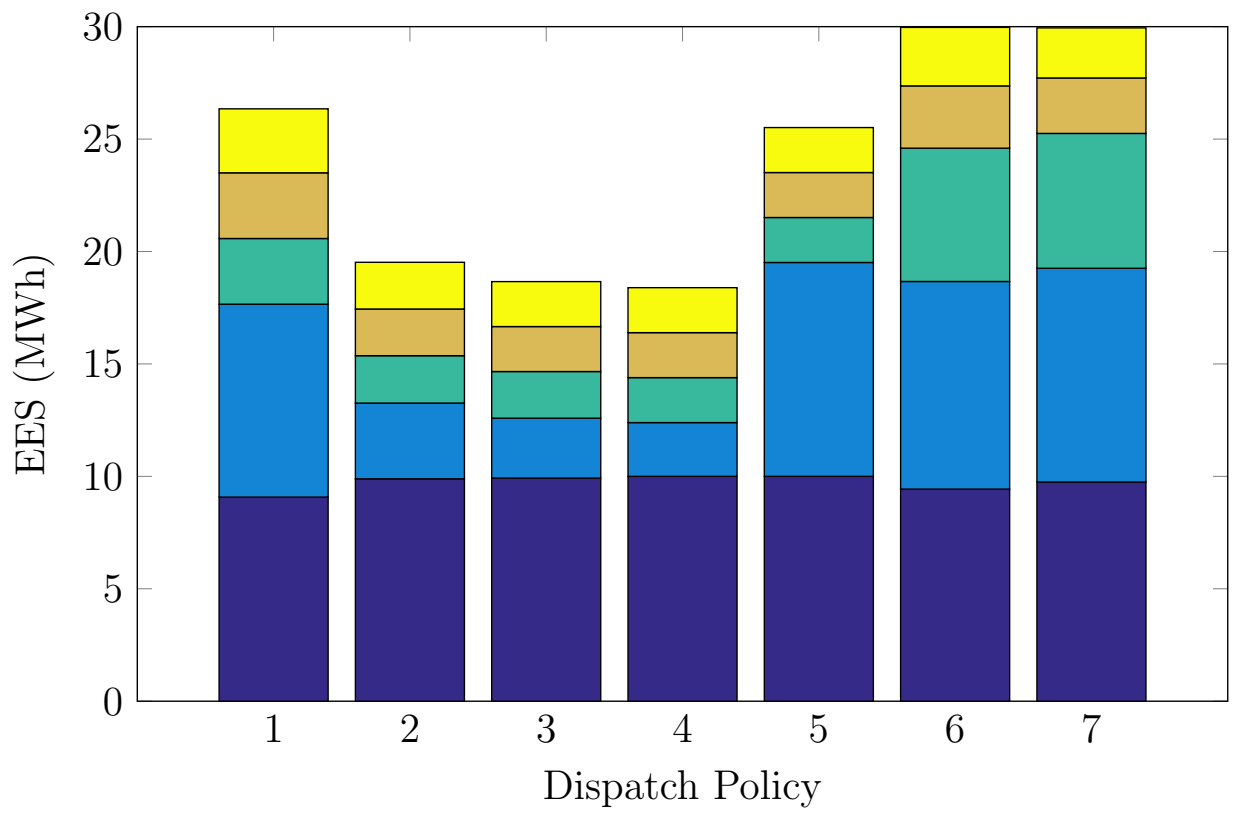

Fig. 4.5 Stacked plot of the expected energy served (EES) by the $\mathcal{I}_{g}$ resources. The resources are stacked upward from the cheapest to the most expensive, starting from the bottom.

Fig. 4.6 plots the expected energy not served (EENS). Clearly, policies 2-4 perform the worst in reducing EENS, whereas policy 1 (enclosing 20\%) outperforms policy 5 (enclosing 99.9\%). Thus, the most stringent envelope (under policy 5 ) does not necessarily result in better EENS values. In contrast, the probabilistic policies 6 and 7 outperform all other policies, in terms of substantially reducing EENS.

On the other hand, Fig. 4.7 plots the expected energy curtailed (EEC). The probabilistic policies 6 and 7 performed the worst in terms of reducing EEC. However, the EEC values are negligible compared to the EENS values. Overall, the probabilistic policies, 6 and 7, outperform all other policies in terms of reducing combined EENS and EEC.

Fig. 4.8 and Fig. 4.9 plot the expected dispatch cost of the $\mathcal{I}_{g}$ resources and the total expected cost (including costs of EENS and EEC), respectively. The poor performance of policies 2-4 can be justified by their quest to reduce the expected dispatch cost of the $\mathcal{I}_{g}$ resources, but end up incurring the highest total expected cost, when factoring in the 


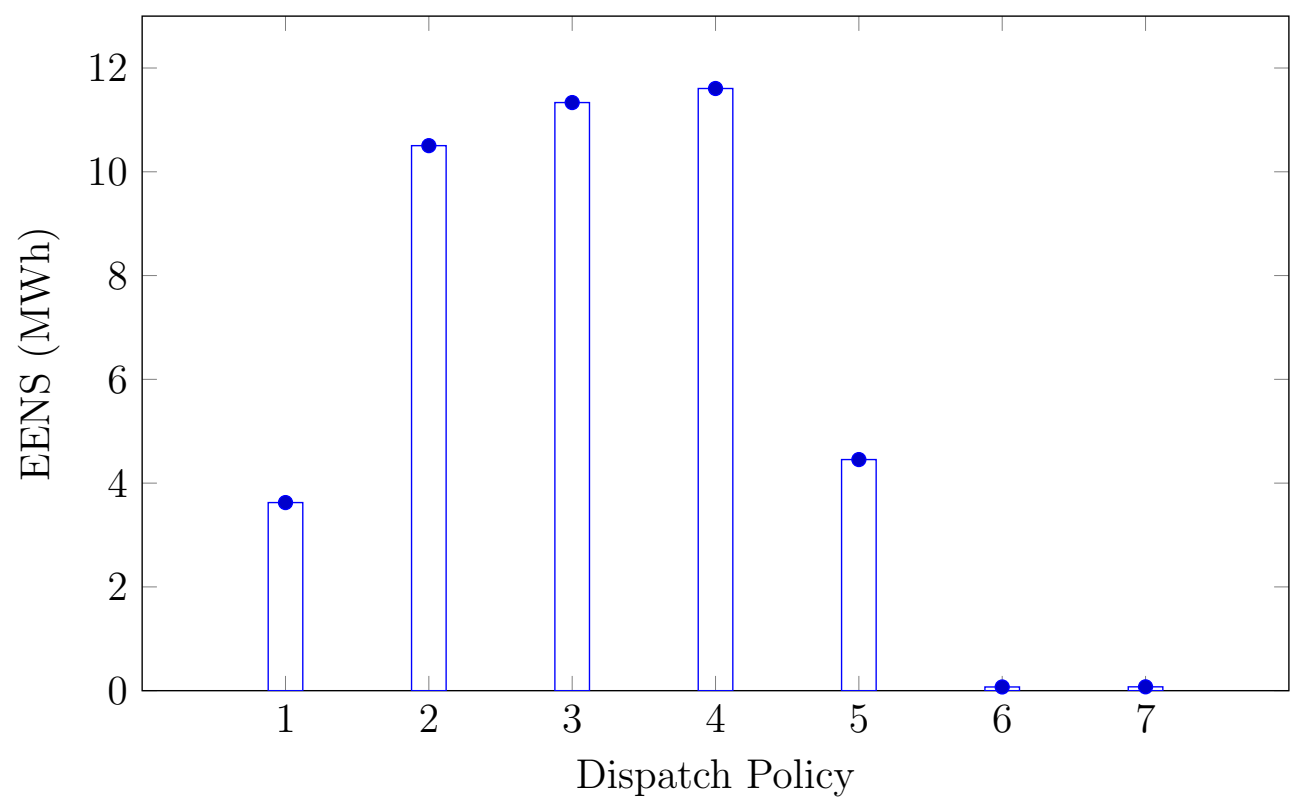

Fig. 4.6 Plot of expected energy not served (EENS).

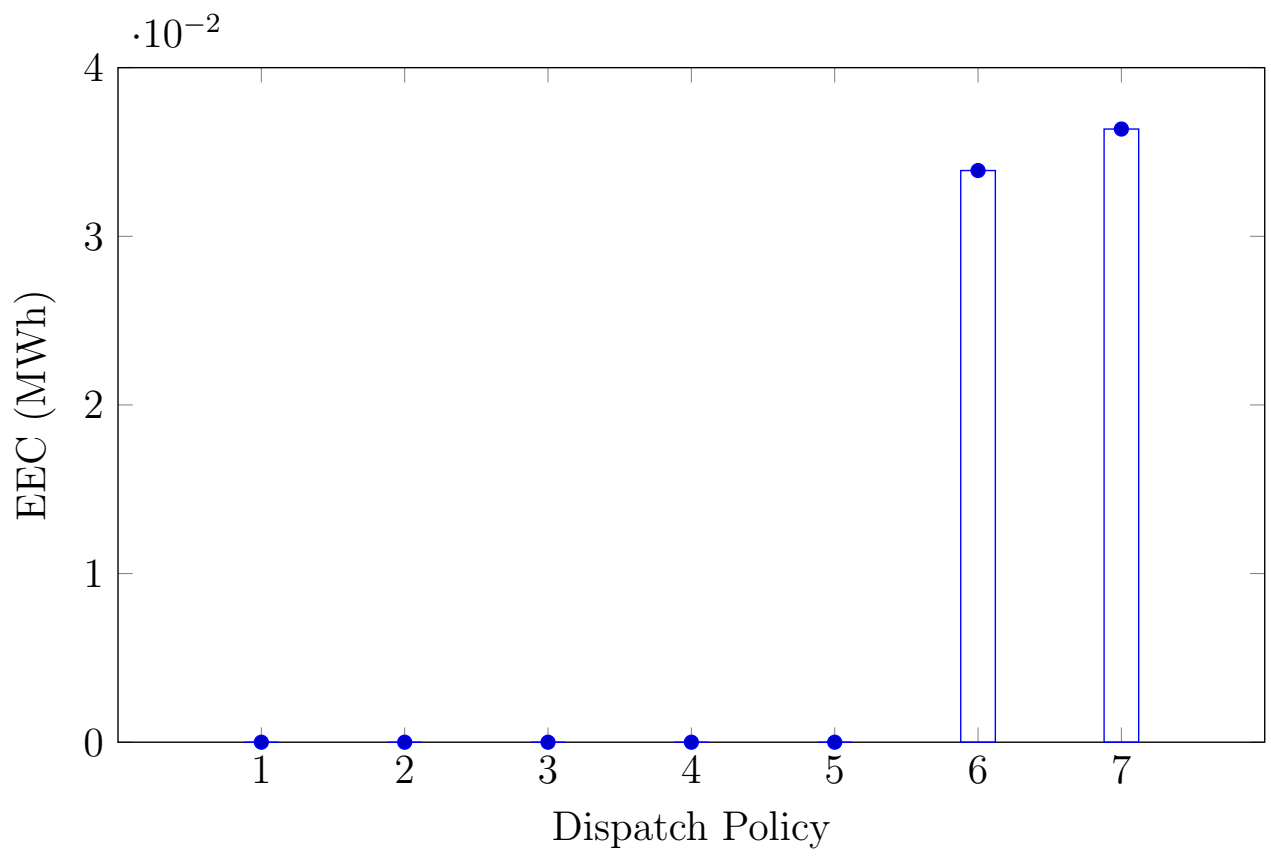

Fig. 4.7 Plot of expected energy curtailed (EEC). 
costs of EENS and EEC. In contrast, policies 1 and 5 favored reducing the total expected cost at the expense of a higher expected dispatch cost. Again, the total expected cost shows that the most stringent envelope (under policy 5) does not necessarily translate to better savings. On the other hand, the probabilistic policies, 6 and 7, outperformed all the other policies in terms of reducing the total expected cost, albeit at the expense of a higher expected dispatch cost.

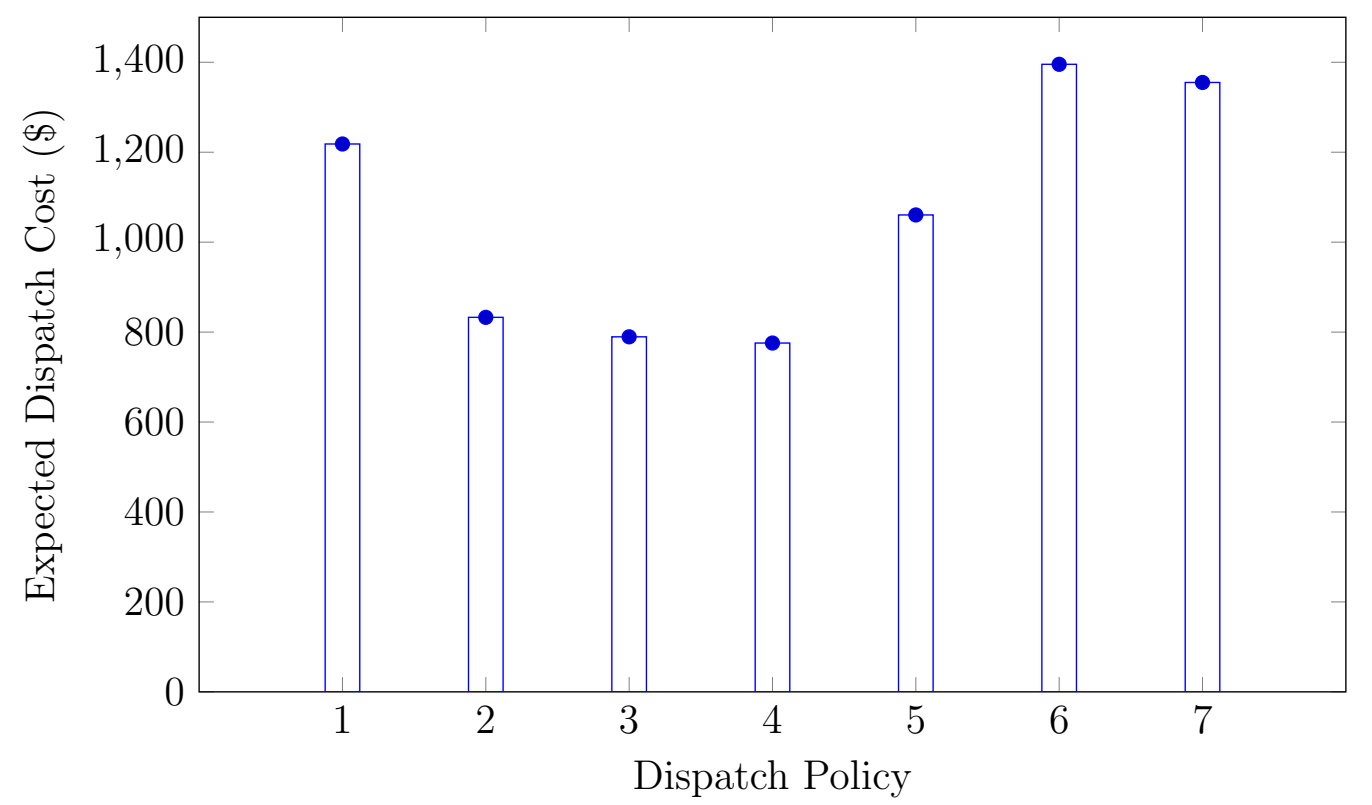

Fig. 4.8 Plot of expected dispatch cost of the $\mathcal{I}_{g}$ resources.

Therefore, there are advantages to probabilistic envelopes planning over planning with a single deterministic envelope. Clearly, the probabilistic policies achieve the best balance between the aforementioned opposing sub-objectives, because they take into consideration the probability distributions enclosed within the envelopes. Finally, there seems to be a noticeable advantage in using the uniform discretization (policy 7) over the non-uniform discretization (policy 6). This tells us that uniformly distributed envelopes (of policy 6) are more effective at capturing the uncertainty, when compared to non-uniformly distributed envelopes (of policy 7). In policy 7, the envelopes are concentrated more towards the center of the Laplace distribution and are not capable of capturing the tails of the net load distribution.

Last but not least, we opted not to solve the described problem via a scenario-based 


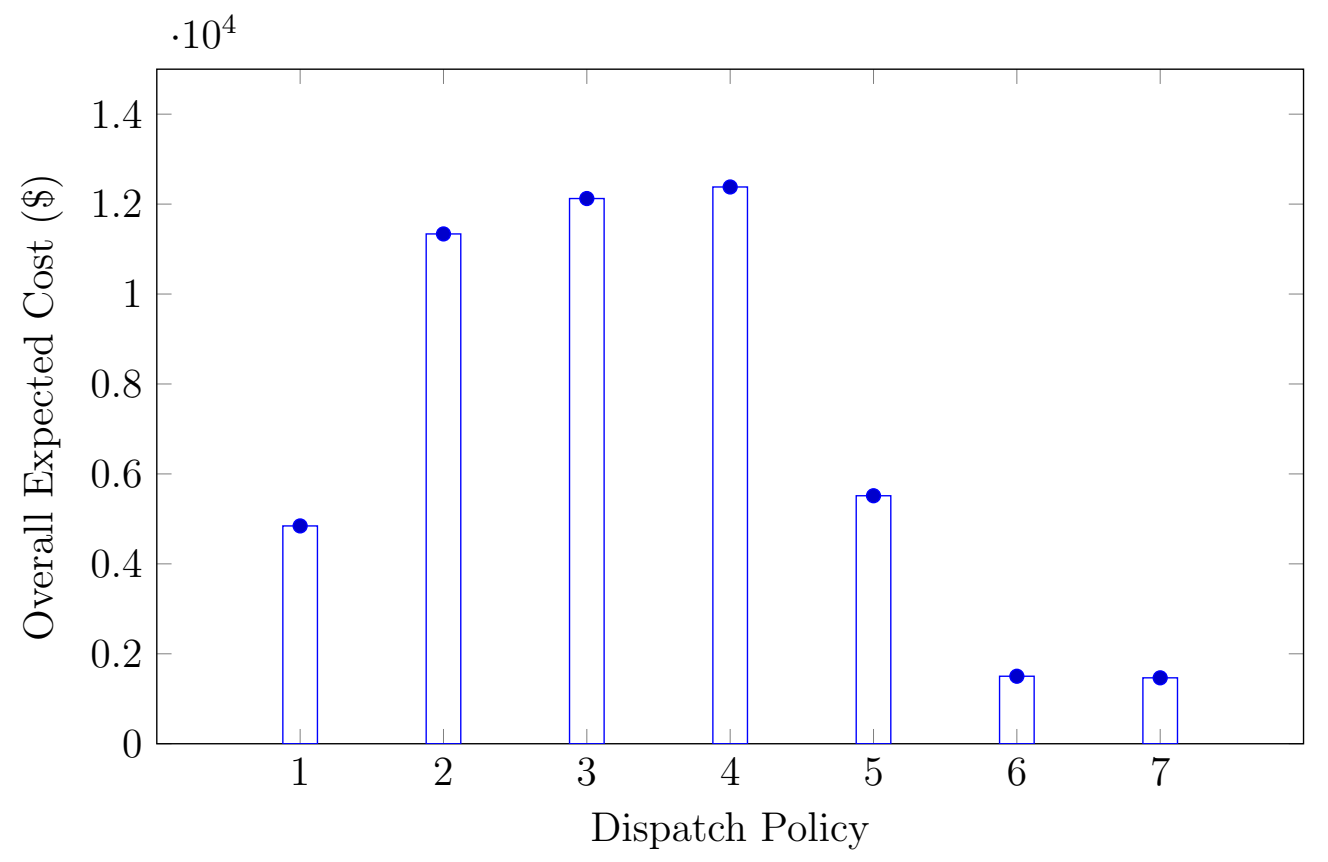

Fig. 4.9 Plot of overall expected cost including costs of EENS and EEC.

stochastic approach. This is because of the intractable computational complexity of modeling the stochastic scenarios over 12 intra-hourly time steps, solving the stochastic program in a receding-horizon fashion, and simulating $J$ realizations of the net load - to collect sufficient data for computing the expected values. Moreover, we can see from the figures that the performance of the probabilistic envelopes approach is very close to the ideal operation-i.e. merit-order dispatch with zero EENS and EEC values. Therefore, we expect the comparison between the probabilistic envelopes approach and the scenario-based stochastic approach to be a close one. After all, the purpose of proposing the probabilistic envelopes approach was to provide a computationally viable alternative to stochastic planning, especially on the intra-hourly timescale involving receding-horizon economic dispatch.

\subsection{Conclusion}

In this Chapter, we proposed a probabilistic planning approach, as an alternative to stochastic optimization for operations planning in a power system with significant proportions of RES. The approach extends Chapter 2 from planning with a single deterministic flexibility requirement envelope to planning with several probability-weighted flexibility requirement 
envelopes. By acknowledging the probability of scenarios enclosed within each envelope, we end up reducing the overall expect costs, as well as improving system reliability via reducing EENS and EEC. The advantage of the proposed probabilistic formulation is circumventing the exorbitant computational costs incurred by stochastic optimization and circumventing the complexity of scenario construction and reduction techniques. 


\section{Chapter 5}

\section{Dynamical Planning With Flexibility Envelopes}

Traditionally, the planning of operating reserve has been done in terms of capacity and average constant ramping requirements, whereas the newly emerging concept of power system flexibility puts emphasis on resources' maneuverability, as well as accurately capturing the intra-hourly variability and uncertainty resulting from significant penetration of renewable power generation. However, the traditional reserve paradigm is deemed impeding to the notion of flexibility, whereas there is yet to be a proper way of defining power system flexibility. To that end, we re-think the fundamental meaning of reserve with respect to the emerging concept of flexibility and present a new flexibility modeling framework. We characterize flexibility provision and flexibility requirements via dynamical envelopes that can reflect the higher-order dynamics of power system resources and those of variability and uncertainty. We assert that flexibility adequacy is directly related to how well the aggregate flexibility envelope formed by flexibility resources encloses the flexibility requirement envelope and its dynamics over operational planning horizons. An optimal flexibility planning problem with envelopes is formulated, followed by examples involving unit commitment and economic dispatch.

\subsection{Introduction}

There is yet to be consensus across industry and academia on the exact definition of operating reserve [62], let alone defining the emerging concept of power system flexibility [5]. 
The consensus, however, is that operating reserve needs to be flexible enough to deal with the variability and uncertainty arising from the integration of renewable energy sources (RES). Still, the traditional reserve paradigm permeates the state-of-the-art research on power system flexibility and restricts flexibility to capacity and average constant ramping assumptions. In contrast, we envision power system flexibility to be more dynamic, as the trajectories of resources and the net load maneuver over operational horizons. The higherorder dynamics of these trajectories may contain valuable information about flexibility provision (coming from resources) and flexibility requirements (coming from the net load, i.e. load less non-dispatchable generation). Thus, to expose such higher-order flexibility features, there is a need to move beyond the traditional paradigm.

In Chapter 1, we performed an extensive review of several key papers within the area of power system flexibility and its operational scheduling; see [50, 63, 25, 9, 16, 23] among many others for some of the most relevant contributions. In Chapter 2, we proposed the concept of flexibility envelopes to capture and manage intra-hourly reserve requirements as an alternative approach to traditional operating reserve scheduling methods in the presence of significant renewable power generation. The proposed concept permitted us to preposition dispatchable resources ahead of time, such that their aggregate flexibility envelope encloses - over the operational horizon - the flexibility requirement envelope arising from the net load's variability and uncertainty. By doing so, the power system was guaranteed to have sufficient flexibility to provide frequency control and load following on all intra-hourly time scales, not just the ten-minute (primary and secondary reserves) and the sixty-minute (tertiary reserve) durations. The example provided illustrated the advantage of the proposed flexibility envelope paradigm, by reducing the expected energy not served and the expected energy curtailed, as well as by tightening their empirical distributions. The lesson to take from this work was that power system flexibility should not be limited to the traditional reserve types. It is rather a spectrum of reserve requirements spanning the full range of intra-hourly time scales, which transcends the traditional paradigm.

In this chapter, we extend the flexibility envelopes concept by characterizing operating reserve dynamically. Frequency control and load following are cast from the view that power system resources should have sufficient collective flexibility to track well enough all plausible net load trajectories. For this, we take a time-domain trajectorial approach to quantifying the dynamical behavior of flexibility requirements and flexibility provision over operational horizons. We use envelopes to bound all plausible realizations of the net 
load random process and all feasible output trajectories of a power system resource (or an aggregate of resources). We assert, by enclosing (tracking) the net load's envelope (i.e., the flexibility requirement envelope), trackability of all bounded realizations of the net load random process is guaranteed; this constitutes an adequate level of system flexibility.

Through this proposal, the extended concept of flexibility envelopes is set to replace classical reserve types with a continuous spectrum of reserve, thus alleviating the lack of consensus mentioned earlier. In fact, regulation and load-following actions occur simultaneously as resources change their output levels in real time, thus making the temporal separation between the different reserve types unwarranted, especially for practical timeinvariant power system planning problems. As a side-benefit of the envelope tracking approach, we come to propose a flexibility-constrained mixed-integer linear unit commitment formulation which can model adequately the dynamics of power system resource ramping processes, in comparison to constant maximum ramping assumptions regularly found in the state-of-the-art, e.g., [64]. Moreover, experimental results show how the dynamic flexibility envelope tracking approach is more cost effective at both the unit commitment and economic dispatch stages. It does so while still providing the similar levels of system reliability provided by traditional unit commitment and economic dispatch approaches.

\subsection{Preliminaries}

\subsubsection{Variability vs Uncertainty}

In reference to [20], variability arises because the net load has a maximum output limit that changes with time, whereas uncertainty arises because this maximum limit is unknown with perfect accuracy. Alternatively, we redefine variability and uncertainty in line with the trajectorial analysis presented in this chapter. Essentially, the two definitions are the same; the former looking at a certain point in time, whereas the latter looking at it as a function of time.

The net load random process is a collection of real-valued functions (realizations) that are parameterized by time $\tau$. Fig. 5.1 shows several realizations, $v(\tau ; k)$, of the net load random process over the horizon $\tau \in[0, H]$, as seen from the present time $k(\tau=0)$. Uncertainty is defined by the fact there are many plausible realizations the net load random process can take on, whereas variability is defined by the time-varying behavior of each of 
the realizations, as they evolve over the operational horizon.

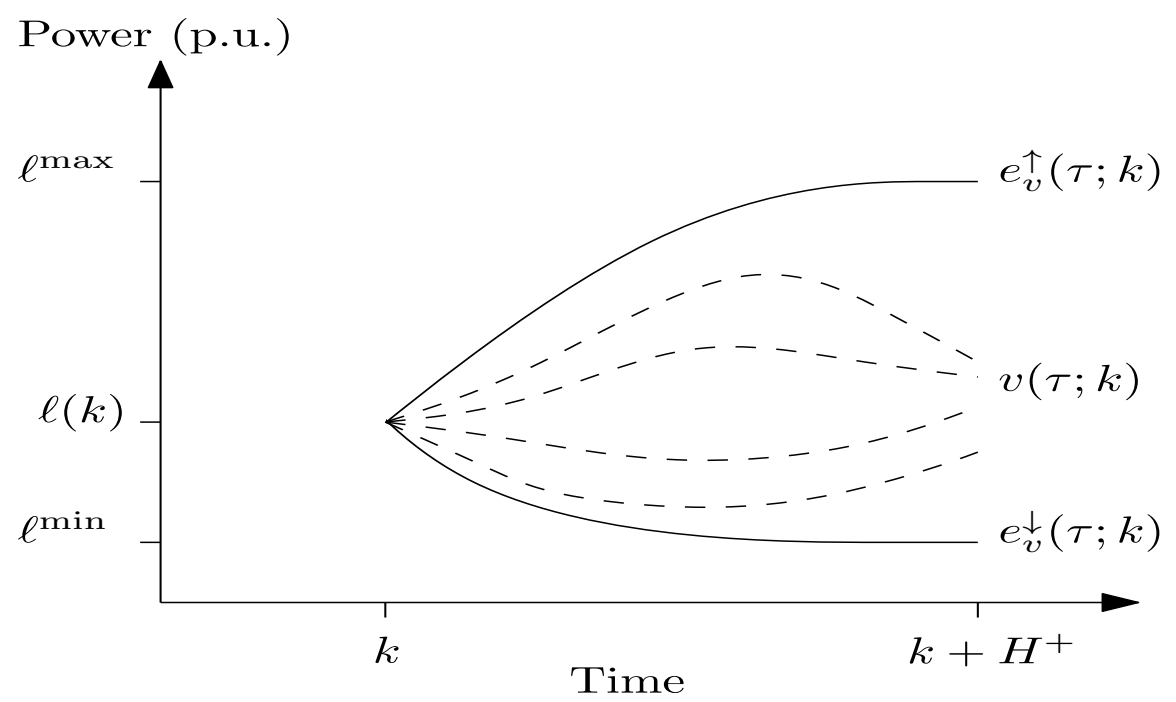

Fig. 5.1 Schematic illustration of net load variability and uncertainty.

The flexibility requirement envelope is defined such that it encloses all plausible realizations of the net load random process (i.e. uncertainty), as well as the $n^{\text {th }}$-order dynamics that capture the time-varying behavior of each of the realizations (i.e. variability). The envelope is represented by the trajectories $e_{v}^{\downarrow}(\tau ; k)$ and $e_{v}^{\uparrow}(\tau ; k)$ in Fig. 5.1. The envelope's trajectories, themselves, are realizations of the net load random process and exhibit maximum magnitude of deviation from $\ell(k)$, the net load forecast level.

\subsubsection{Relevance to Tracking Theory}

Fundamentally, reserve is deployed to nullify deviations in the system frequency by restoring and maintaining balance between supply and demand. Ideally, frequency deviations should never occur if the net load is tracked perfectly by the aggregate response of resources. That is, perfect tracking is achieved when the aggregate response of resources is capable of producing a trajectory equal to the net load realization over the operational horizon. Therefore, we premise here that power system flexibility is essentially a measure of trackability - the ability of the aggregate response of resources to track any realization of the net load random process over the operational horizon.

In order to achieve trackability of all net load realizations, the aggregate response of available power system resources must be capable of matching the whole range of $n^{t h}$-order 
derivatives pertaining to all realizations of the net load random process. For instance, consider two trajectories, $x_{1}(\tau)$ and $x_{2}(\tau)$. They are said to be equal over the horizon $\tau \in[0, H]$, if they start at the same level:

$$
x_{1}(0)=x_{2}(0)
$$

and their $n^{\text {th }}$-order derivatives are equal:

$$
\frac{d^{n}}{d \tau^{n}} x_{1}(\tau)=\frac{d^{n}}{d \tau^{n}} x_{2}(\tau) \quad \forall \tau \in(0, H)
$$

This information can be compactly summarized by the flexibility requirement envelope and the aggregate flexibility envelope of resources. Our assertion here is that enclosing the flexibility requirement envelope by the aggregate flexibility envelope of resources will achieve trackability of all net load realizations bounded by the flexibility requirement envelope, thus guaranteeing adequate system flexibility.

In comparison, the current approach in power system operation is that resources move from one operating point to another in an affine (average linear) ramping fashion. This can be regarded as a relaxation (or approximation) of the stringent trackability premise adopted here. Average ramping appears because resources are required to ramp linearly to the 10minute (primary and secondary) and the 60-minute (tertiary) reserve levels within 10 and 60 minutes, respectively, on average regardless of the intra-hourly behavior. Whether or not such practices remain viable under high variability and uncertainty - in modern power systems admitting increasing levels of RES - should be the subject of further research. For instance, one may ask what would be the impact of discarding higher-order dynamics on the ability of operating reserve to nullify frequency deviations in due time, so as not to violate the standard reliability criterion. The proposed flexibility assessment framework should facilitate answering such questions in the future. Finally, we underline that in practice perfect tracking will never be possible and, as a result, frequency excursions would continue to happen in real time operation. What we propose here, however, should contribute in reducing their prevalence and their magnitude through more appropriate representations of net load and power system resource dynamics. 


\subsubsection{State-Space Interpretation}

Time-invariant power system resources and stationary net load random processes are implicitly independent of time, thus permitting the use of state-space methods in defining the notion of flexibility. Equally important, it emphasizes on the time-independent characterization of flexibility, as opposed to the time-dependent categorization of operating reserves used currently. This constitutes a major departure from the current interpretations and understandings. However, we argue that this new interpretation should be beneficial in how it can describe more simply the dynamic capabilities of reserve resources, which are driven by the dynamic requirements of net load variability and uncertainty.

Consider, for instance, the state vector as a tuple of $n^{\text {th }}$-order derivatives - capacity being the zeroth-order - in $\mathbb{R}^{n}$. Looking at Fig. 5.2, the reachable set in $\mathbb{R}^{n}$ of a power system resource at $\tau$, where $\tau \in\left[0, H^{+}\right]$, can be constructed by considering all states visited from $k$ up till $k+\tau$, traversing all feasible trajectories forward in time. This assesses flexibility delivery. Likewise, the controllable set in $\mathbb{R}^{n}$ at $\tau$, where $\tau \in\left[-H^{-}, 0\right]$, can be constructed by considering all the states visited from $k$ up till $k-\tau$, traversing (extending) all feasible trajectories backward in time. This assesses flexibility recovery or regeneration. The same can be said for the net load random process by considering all its plausible realizations both forward and backward in time. Here, the net load random process is assumed to be stationary, in comparison to the time-invariance property of a power system resource.

Henceforth, trackability implies that at every $\tau$, where $\tau \in\left[0, H^{+}\right]$, the reachable set of the aggregate response of the available power system resources is a superset of the reachable set of the net load random process, and that at every $\tau$, where $\tau \in\left[-H^{-}, 0\right]$, the controllable set of the aggregate response of resources is a superset of the controllable set of the net load random process, given the same initial conditions at $\tau=0$.

\subsection{The Flexibility Envelope of a Power System Resource}

Before we begin, we let the index $k$ correspond to the actual operational time, whereas the index $\tau \in\left[-H^{-}, H^{+}\right]$corresponds to the envelope's evolution time (i.e. the receding horizon [65]). $H^{-}$is chosen such that the backward-in-time evolution of the envelope is sufficient to reach the minimum and maximum output levels, whereas $\mathrm{H}^{+}$is chosen such 


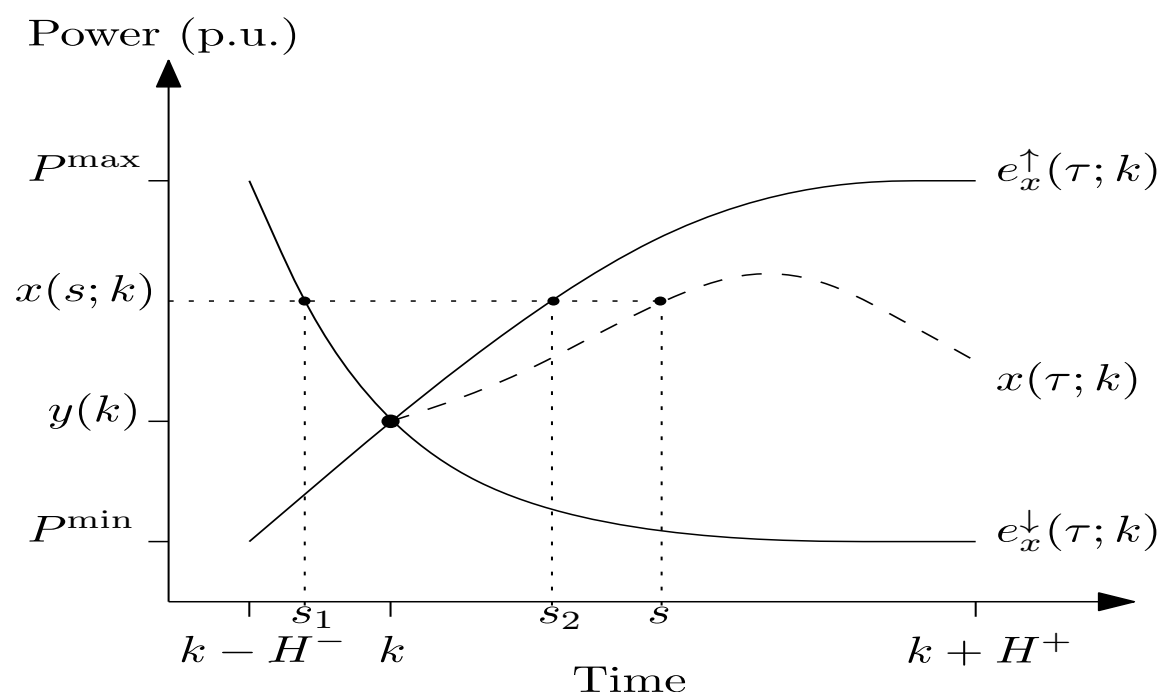

Fig. 5.2 Schematic illustration of the flexibility envelope of a resource.

that the forward-in-time evolution of the envelope is sufficient to reach the minimum and maximum output levels, as well.

Reserve is deployed when the actual output of a power system resource over the horizon deviates from the present output level, $y(k)$. Looking at Fig. 2.4, let $x(\tau ; k)$ represent a feasible output trajectory of a power system resource over the horizon $\tau \in\left[0, H^{+}\right]$, as seen from the present time $k$, where $x(0 ; k)=y(k)$. Here, we assume that $x(\tau ; k)$ is $n$ times continuously differentiable on the interval $\tau \in\left[0, H^{+}\right]$. Thus, the set of all feasible trajectories, $x(\tau ; k) \in \mathcal{X}$, represents the flexibility potential of a power system resource over the horizon $\tau \in\left[0, H^{+}\right]$.

There exist two feasible trajectories $e_{x}^{\uparrow}(\tau ; k) \in \mathcal{X}$ and $e_{x}^{\downarrow}(\tau ; k) \in \mathcal{X}$ that constitute the flexibility envelope of a power system resource over the horizon $\tau \in\left[0, H^{+}\right]$. Looking at Fig. 2.4, the envelope also extends backward in time from $\tau=0$ to $\tau=-H^{-}$. The half-envelope $e_{x}^{\uparrow}(\tau ; k)$ evolves monotonically upward whereas the half-envelope $e_{x}^{\downarrow}(\tau ; k)$ evolves monotonically downward as $\tau$ varies from $-H^{-}$to $H^{+}$. The envelope's evolution over $\tau \in\left[0, H^{+}\right]$assesses the ability to ramp away from the present output level $y(k)$, whereas the envelope's evolution over $\tau \in\left[-H^{-}, 0\right]$ assesses the ability to ramp back to the present output level $y(k)$. That is, ramping away from $y(k)$ constitutes flexibility generation/delivery, whereas ramping back to $y(t)$ constitutes flexibility regeneration/recovery.

The two half-envelopes must cross each other at $y(k)$, when $\tau=0$, thus satisfying the 
initial condition

$$
e_{x}^{\downarrow}(0 ; k)=e_{x}^{\uparrow}(0 ; k)=y(k)
$$

The envelope must also be able to reach and come back from the maximum/minimum output levels, thus satisfying the boundary conditions

$$
\begin{aligned}
& e_{x}^{\downarrow}\left(-H^{-} ; k\right)=e_{x}^{\uparrow}\left(H^{+} ; k\right)=P^{\max } \\
& e_{x}^{\uparrow}\left(-H^{-} ; k\right)=e_{x}^{\downarrow}\left(H^{+} ; k\right)=P^{\min }
\end{aligned}
$$

The flexibility envelope of a power system resource must enclose its flexibility potential $\mathcal{X}$. That is, for any feasible trajectory $x(\tau ; k) \in \mathcal{X}$, its $n^{t h}$-order derivatives are bounded, from above and from below, by the $n^{\text {th }}$-order derivatives of the upward $e_{x}^{\uparrow}(\tau ; k)$ and downward $e_{x}^{\downarrow}(\tau ; k)$ flexibility half-envelopes, respectively. To see this, as we refer to Fig. 2.4, we pick a point on the trajectory $x(\tau ; k)$ at $\tau=s$ and draw a horizontal line intersecting the downward and upward flexibility half-envelopes at $\tau=s_{1}$ and $\tau=s_{2}$, respectively. If the trajectory $x(\tau ; k)$ is moving downward at $\tau=s$, its $n^{\text {th }}$-order derivatives evaluated at $\tau=s$ are bounded from below by those of the downward flexibility half-envelope $e_{x}^{\downarrow}(\tau ; k)$, evaluated at $\tau=s_{1}$ :

$$
\left.\frac{d^{n}}{d \tau^{n}} x(\tau ; k)\right|_{\tau=s} \geq\left.\frac{d^{n}}{d \tau^{n}} e_{x}^{\downarrow}(\tau ; k)\right|_{\tau=s_{1}}
$$

On the other hand, if the trajectory $x(\tau ; k)$ is moving upward at $\tau=s$, its $n^{\text {th }}$-order derivatives evaluated at $\tau=s$ are bounded from above by those of the upward flexibility half-envelope $e_{x}^{\uparrow}(\tau ; k)$, evaluated at $\tau=s_{2}$ :

$$
\left.\frac{d^{n}}{d \tau^{n}} x(\tau ; k)\right|_{\tau=s} \leq\left.\frac{d^{n}}{d \tau^{n}} e_{x}^{\uparrow}(\tau ; k)\right|_{\tau=s_{2}}
$$

We stress that the comparison of the $n^{\text {th }}$-order derivatives is carried out by drawing a horizontal line intersecting the trajectory and the flexibility half-envelopes at the same output level. It is not carried out by drawing a vertical line intersecting the trajectory and the flexibility half-envelopes at the same time instant. This re-emphasizes the timeindependent characterization of flexibility that we seek to achieve for time-invariant power system planning. This is where we depart from the traditional time-based characterization 
of operating reserve. Finally, the envelope's equations, themselves, are feasible trajectories of the power system resource. Any two feasible trajectories that do not enclose the flexibility potential are not proper candidates for defining a resource's flexibility envelope.

\section{Example}

Consider a power system resource modeled as a first-order linear time-invariant system:

$$
\begin{array}{r}
10 \frac{d}{d \tau} x(\tau)+x(\tau)=f(\tau) \\
0 \leq f(\tau) \leq 10 \mathrm{MW}
\end{array}
$$

where $x(\tau)$ is the power output trajectory, and $f(\tau)$ is a trajectory of the resource's power output set point. Thus, given its capacity constraints, the flexibility potential of the resource, as represented by the shaded region in the phase-plane of Fig. 5.3, is bounded by the two maximal phase-plane trajectories

$$
\begin{aligned}
& \frac{d}{d \tau} e_{x}^{\uparrow}(\tau)=1-\frac{e_{x}^{\uparrow}(\tau)}{10} \\
& \frac{d}{d \tau} e_{x}^{\downarrow}(\tau)=-\frac{e_{x}^{\downarrow}(\tau)}{10} \\
& 0 \leq e_{x}^{\uparrow \downarrow}(\tau) \leq 10 \mathrm{MW}
\end{aligned}
$$

Note that $\tau$ appears only as a parameter in the equation, which implies that flexibility characteristics are implicitly independent of time.

By inspection of Fig. 5.3, a power system resource can operate at any point within the shaded parallelogram. For instance, the phase-plane representation says that a resource operating at a low output can ramp correspondingly faster than a resource already operating near its maximum power output. This kind of representation is commensurate of power system resources responding as first-order lags to step changes to their power output set points. Moreover, this representation is closer to the reality of physical power system resources (thermal generators, especially) as it accounts for the natural dynamic response of resources to changes in their power set points. ${ }^{1}$

\footnotetext{
${ }^{1}$ In the general case where the restrictions on the states of the system included orders of $n \geq 2$, one would establish a similar phase-space region within which the resource's state could evolve.
} 
Given, for instance, that $x(k)=3 \mathrm{MW}$ at $\tau=0$, we integrate along the two maximal phase-plane trajectories, both forward and backward in time, to yield the time-domain flexibility envelope of the power system resource shown in Fig. 5.4. To show how the flexibility envelope encloses all flexibility deployment trajectories by the resource, we solve (5.8) for randomly generated $f(\tau)$ trajectories, given $x(k)=3 \mathrm{MW}$ at $\tau=0$. As shown in Fig. 5.4, the simulated trajectories of flexibility deployment are enclosed, both in capacity and firstorder ramping, by the flexibility envelope of the resource. The simulated trajectories are jittery due to the first derivative of $f(\tau)$ being unconstrained. Thus, this leads to unconstrained second-order derivatives for $x(\tau)$, for which the lack of modeling the second-order derivative may overestimate the ramping capability of the flexibility resource in real time. Finally, when plotting the simulated trajectories in the phase-plane of Fig. 5.3, they should remain within the shaded parallelogram.

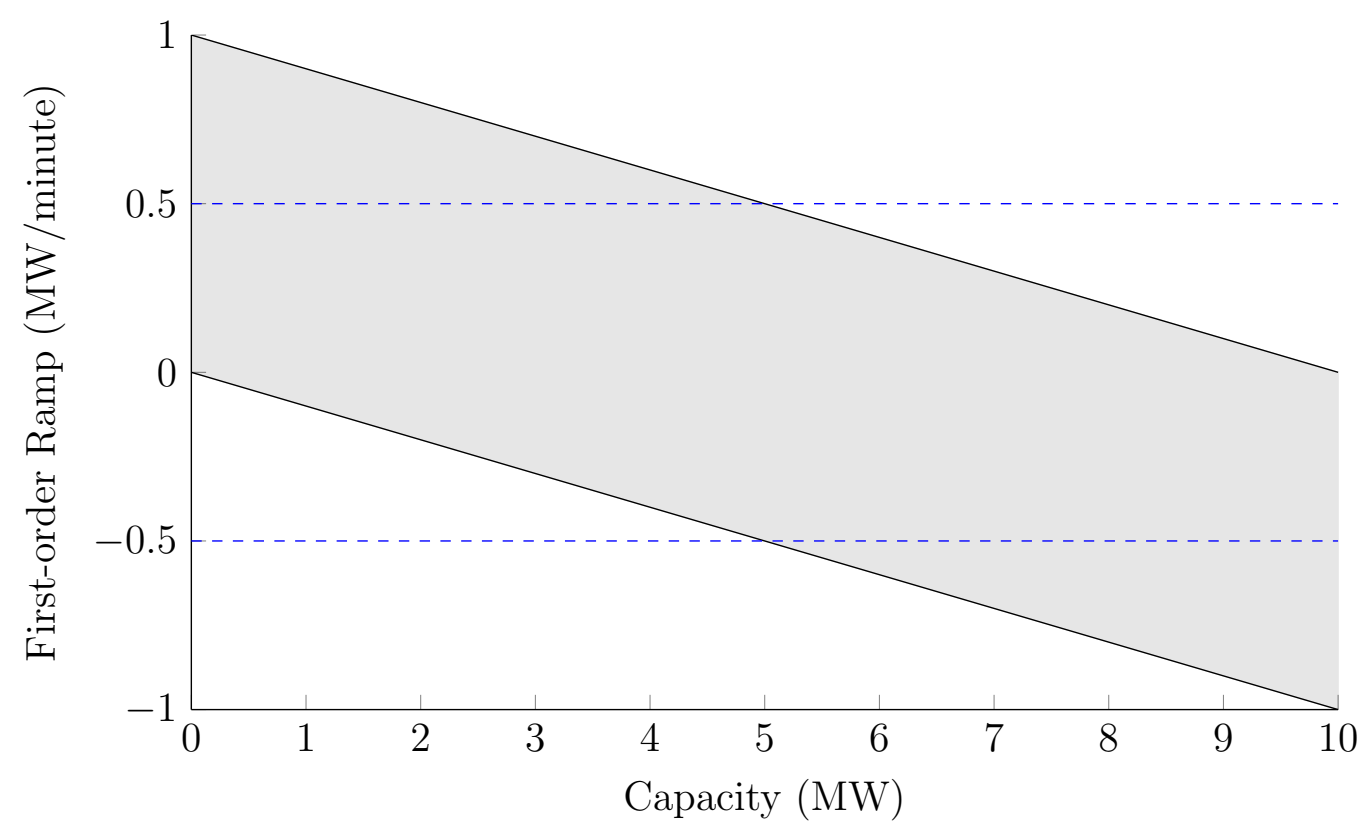

Fig. 5.3 Phase-plane characteristics of a power system resource, indicating the range of ramp rates the resource can sustain ( $y$-axis) depending on its output level ( $x$-axis).

In addition, the dashed envelope lines in Fig. 5.4 represent the same resource given a maximum constant (average) ramping assumption, which is also illustrated by the dashed phase-plane lines in Fig. 5.3. Such operations are deemed less flexible and could subject the resource to physical stress and potential damage when corresponding operating points lie 


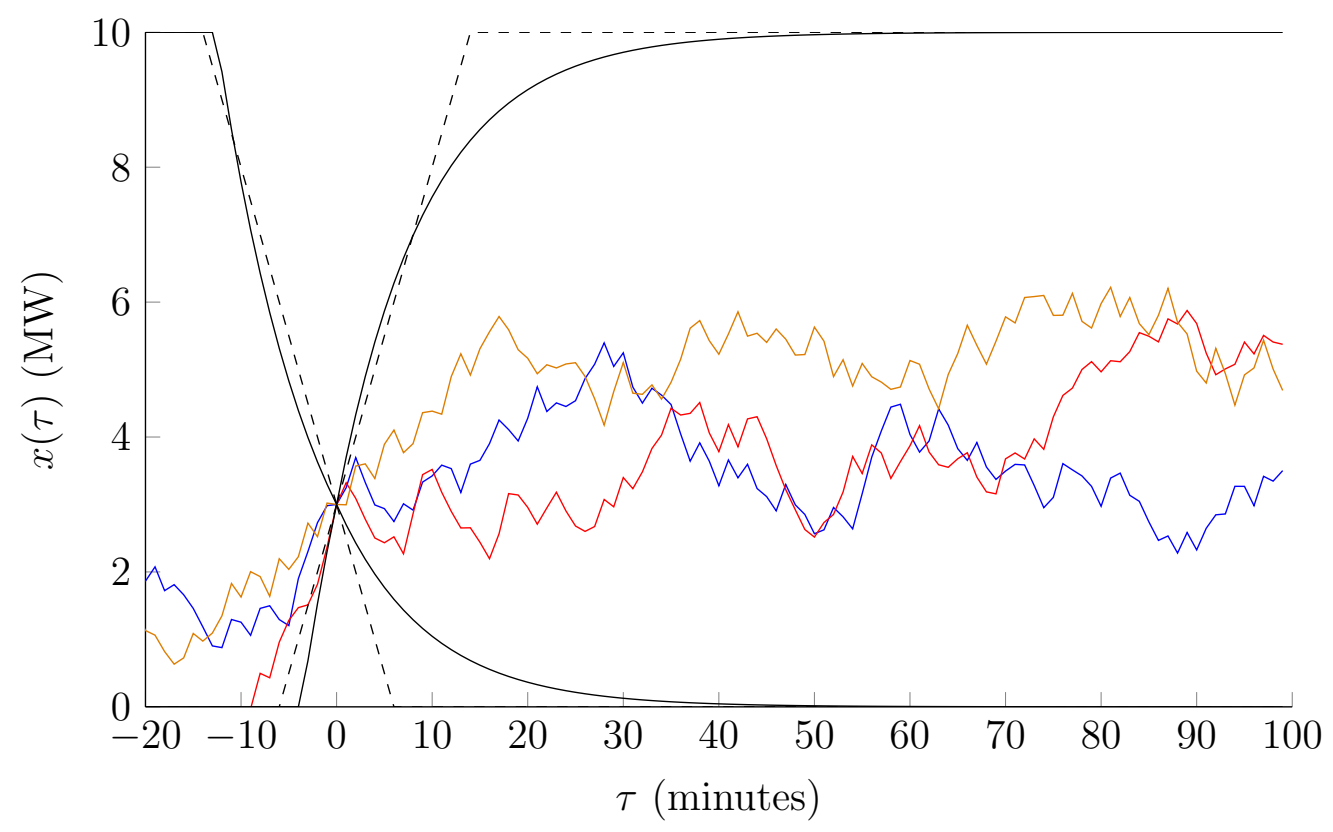

Fig. 5.4 Flexibility envelope of a power system resource.

outside of the shaded parallelogram of Fig. 5.3. We refer the interested reader to [66], which addressed extra wear and tear cost implications of generation ramping processes. Later on, we will show how the use of a dynamic ramp profile can, in fact, provide more flexibility and be more cost-effective than the use of a maximum constant ramp assumption.

\subsection{The Envelope of Flexibility Requirements}

Let $v(\tau ; k)$ represent a realization of the net load random process over the horizon $\tau \in$ $\left[0, H^{+}\right]$, as seen from the present time, such that $v(0 ; k)=\ell(k)$, where $\ell(k)$ is the net load persistence forecast at time $k$. All possible such realizations form the set $\mathcal{V}$ and are assumed to be $n$-times continuously differentiable. Comparing Fig. 5.5 to Fig. 2.4, $v(\tau ; k)$ replaces $x(\tau ; k)$, the subscript $v$ replaces the subscript $x, \ell^{\max }$ replaces $P^{\max }, \ell^{\min }$ replaces $P^{\min }$, and $\ell(k)$ replaces $y(k)$. Avoiding unnecessary repetition, defining the flexibility requirement envelope and envelope enclosure is similar to that of Section III. In other words, the net load is considered as another dynamical resource connected to the grid, albeit partially controllable. Furthermore, we re-emphasize that the flexibility requirement envelope encloses both variability and uncertainty arising from the net load random process. 


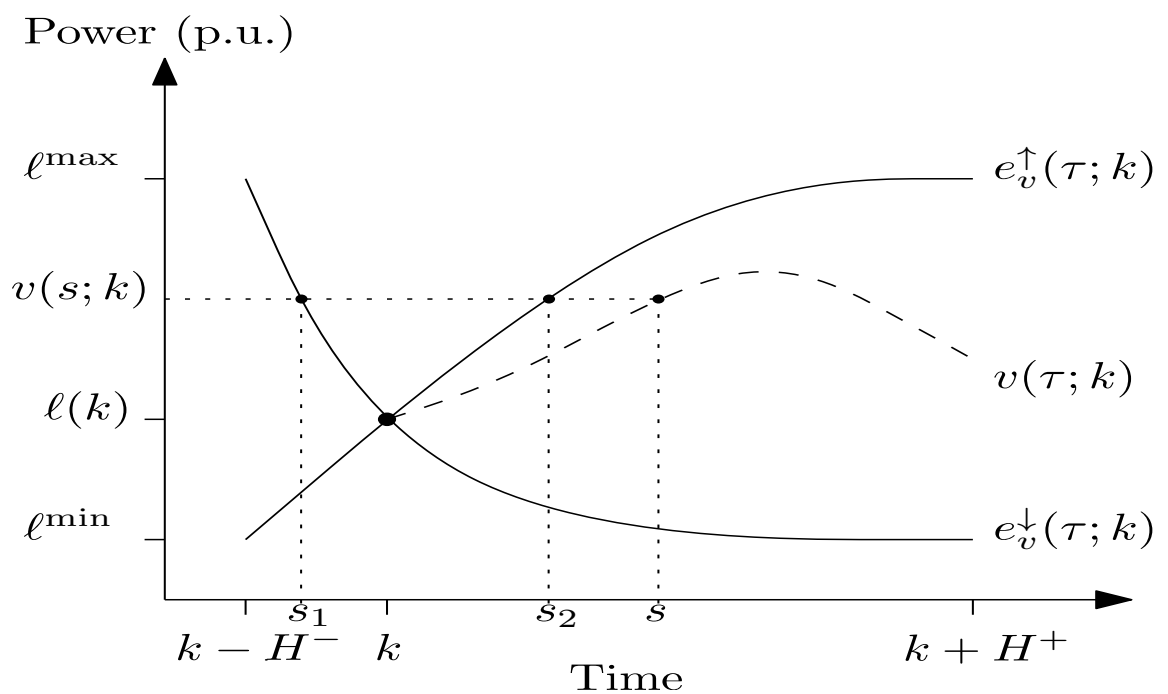

Fig. 5.5 Schematic illustration of the flexibility requirement envelope.

\section{Example}

In this example, we use the load and wind data for the BPA balancing area [59]. There are roughly 89000 samples which are used to compute the net load time series $\ell(k)$, where $k$ is the index of the $k^{t h}$ sample in the dataset. Next, the $n^{t h}$-order forward difference is used to approximate the $n^{\text {th }}$-order derivative at each sample:

$$
\ell^{(n)}(k) \approx \frac{\ell^{(n-1)}(k+1)-\ell^{(n-1)}(k)}{T}
$$

where $T=5$ minutes is the sampling period - the size of the sampling period affects the accuracy of the approximation, as the order of $n$ increases. Fig. 5.6 shows a plot of the tuple $\left(\ell(k), \ell^{(1)}(k), \ell^{(2)}(k)\right)$ using 1000 randomly chosen samples from the dataset, which constitutes a three-dimensional net load dynamical phase-plane.

Furthermore, we compute the $n^{\text {th }}$-order differences for $n=3,4$. Table 5.1 shows the minimum, maximum, mean, and variance of the dataset for $\ell^{(n)}(k)$, where $n=0, \ldots, 4$. The question becomes what is the cutoff order of $n$ beyond which the higher order derivatives can be discarded? To answer this question, next we introduce a preliminary method based on a simplified principal component analysis (PCA) [67]. The goal here is to eliminate the higher-order derivatives having the least impact on the variance in the dataset, where we take the variance in the dataset as a measure of variability and uncertainty in the net load. 


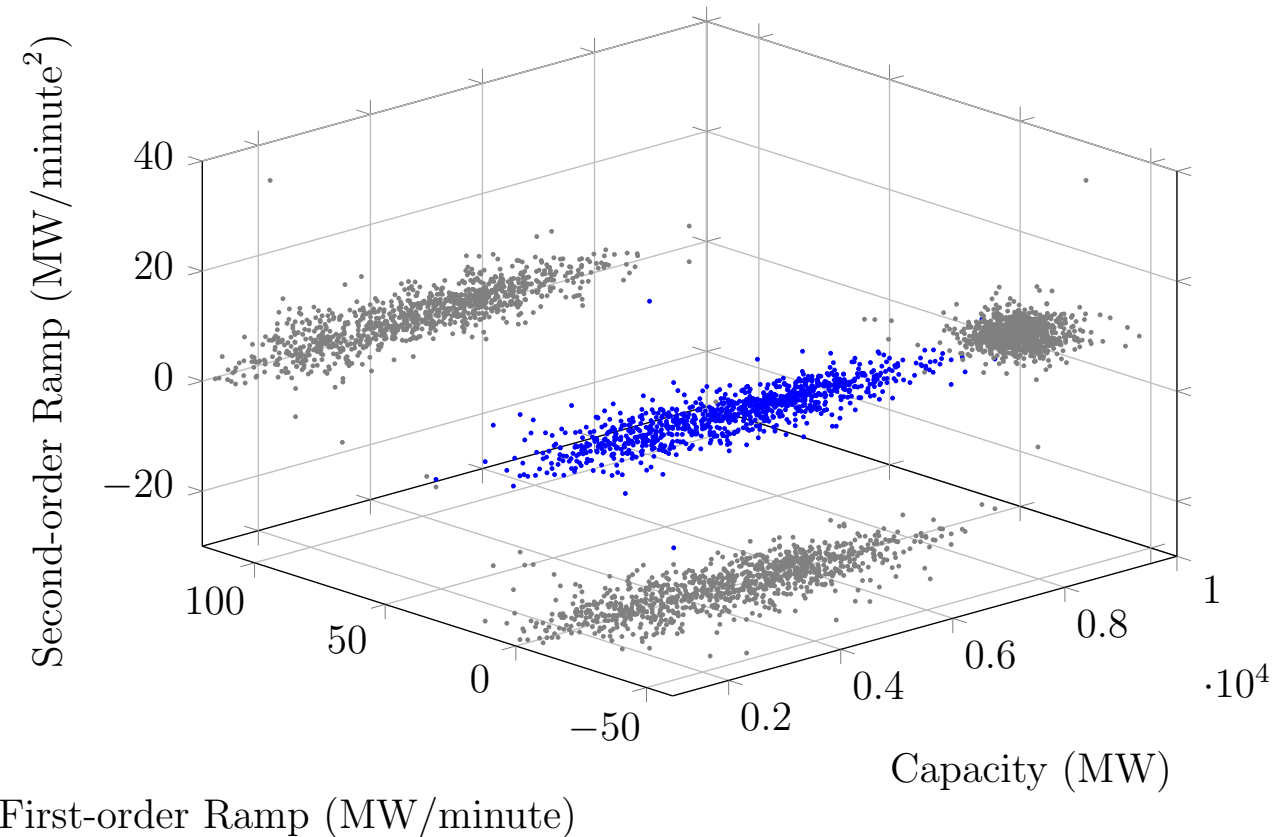

Fig. 5.6 Plot of $\left(\ell(k), \ell^{(1)}(k), \ell^{(2)}(k)\right)$ and their corresponding projections for 1000 randomly chosen samples from the BPA net load dataset.

Table 5.1 Characteristics of $n^{\text {th }}$-Order Differences (Values Are $\times 10^{2}$ )

\begin{tabular}{lccccc}
\hline & $\ell(k)$ & $\ell^{(1)}(k)$ & $\ell^{(2)}(k)$ & $\ell^{(3)}(k)$ & $\ell^{(4)}(k)$ \\
\hline Min & 5.10 & -3.68 & -1.46 & -0.437 & -0.129 \\
Max & 99.2 & 3.64 & 1.08 & 0.446 & 0.176 \\
Mean & 48.9 & $<10^{-4}$ & $<10^{-6}$ & $<10^{-6}$ & $<10^{-7}$ \\
Var. & $2.44 \times 10^{4}$ & 1.41 & 0.101 & 0.0125 & 0.00170 \\
\hline
\end{tabular}


PCA provides a systematic way of reducing the dimensionality of large datasets exhibiting many features (i.e., dimensions). In our case, the rows of the dataset represent the 89000 samples, whereas the columns of the dataset represent the capacity and $n^{\text {th }}$-order derivatives (i.e. the features or dimensions). Thus, PCA ranks the impact of the $n^{\text {th }}$-order derivatives on variance in the dataset, with respect to capacity. Out of the various approaches to PCA, here, we take the eigenvalue decomposition approach, which calculates the eigenvalues of the covariance matrix of the dataset. In the interest of space here, we only show the corresponding eigenvalues

$$
\lambda=\left(\begin{array}{c}
2.44 \times 10^{6} \\
1.46 \times 10^{2} \\
6.30 \times 10^{0} \\
2.52 \times 10^{-1} \\
6.12 \times 10^{-3}
\end{array}\right) .
$$

The eigenvectors of the covariance matrix constitute a new set of orthonormal dimensions that best describe the variance in the dataset, by reducing the noise (information-less) and redundancy (linear dependence) therein [68]. The eigenvectors having the highest corresponding eigenvalues form the principal components (dimensions) of the dataset, whereas the other dimensions may be removed without much loss of information.

The eigenvalues $\lambda$ in (5.14) suggest that the capacity dimension contributes the most to variance in the dataset, whereas the first-order ramp contributes the most to variance, with respect to the other higher-order ramps. Therefore, we choose $n=1$ as the cutoff order and we shall discard the rest for the remaining portions of our discussion. Note, however, that this is a feature of the current dataset being used here. Other net load datasets may warrant the use of more dimensions, yet this is probably unlikely given the time constants of most large wind turbines, for example. On other hand, this may not be the case in solar photovoltaics-dominated systems.

Fig. 5.7 shows a scatter plot in the phase plane of the capacity $(n=0)$ and first-order difference $(n=1)$ of the BPA dataset. In the spirit of the proposal here, one can fit a phase plane envelope to enclose the vast majority of observed capacity-ramp realizations. This envelope would then translate into the time domain into a flexibility requirement envelope which should enclose the vast majority of possible time domain realizations of the 
net load process. For the purpose of this example, the phase plane envelope was fitted to two quadratic polynomials (red lines) to cover the dataset scatter plot. The corresponding polynomials are

$$
\begin{aligned}
& \frac{d}{d \tau} e_{v}^{\uparrow}(\tau)=-1.73\left(e_{v}^{\uparrow}(\tau)-4500\right)^{2}+35 \\
& \frac{d}{d \tau} e_{v}^{\downarrow}(\tau)=1.94\left(5250-e_{v}^{\downarrow}(\tau)\right)^{2}-35 \\
& 1000 \mathrm{MW} \leq e_{v}^{\uparrow \downarrow}(\tau) \leq 9000 \mathrm{MW}
\end{aligned}
$$

In reality, more systematic approaches based on the empirical probability distribution of the dataset cluster are desired; this, however, is left to future research. Moreover, it remains that the resulting envelope corresponding to net load observations has to be updated with time to reflect changes in the power system's generation mix, the topology of its network and/or operational practices.

Given $\ell(k)=5000 \mathrm{MW}$ at $\tau=0$, the flexibility requirement envelope is generated by integrating along the phase-plane polynomials, starting at $\ell(k)=5000$ MW. Fig. 5.8 plots the flexibility requirement envelope (solid lines), as well as randomly chosen sample trajectories from the dataset passing through $5000 \mathrm{MW}$. As shown, the flexibility requirement envelope encloses the capacity and first-order ramping of the sample trajectories. Moreover, it takes about 400 minutes for the envelope to reach maximum variability.

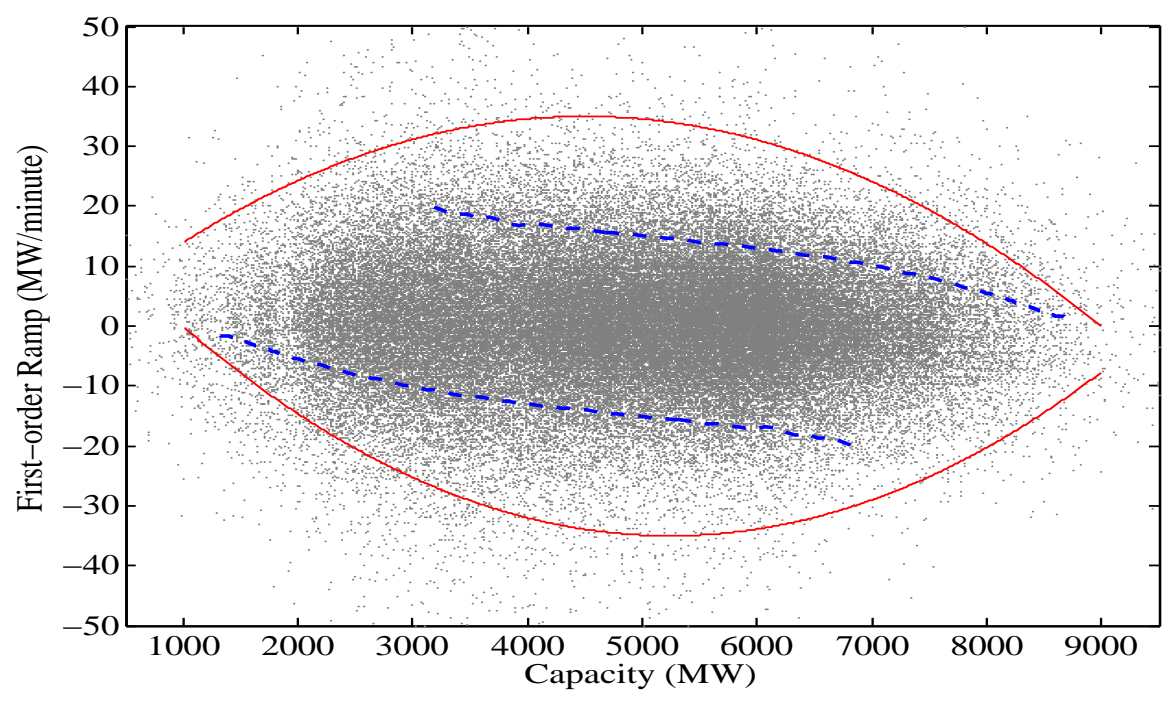

Fig. 5.7 Phase-plane characteristics of BPA's net load dataset. 


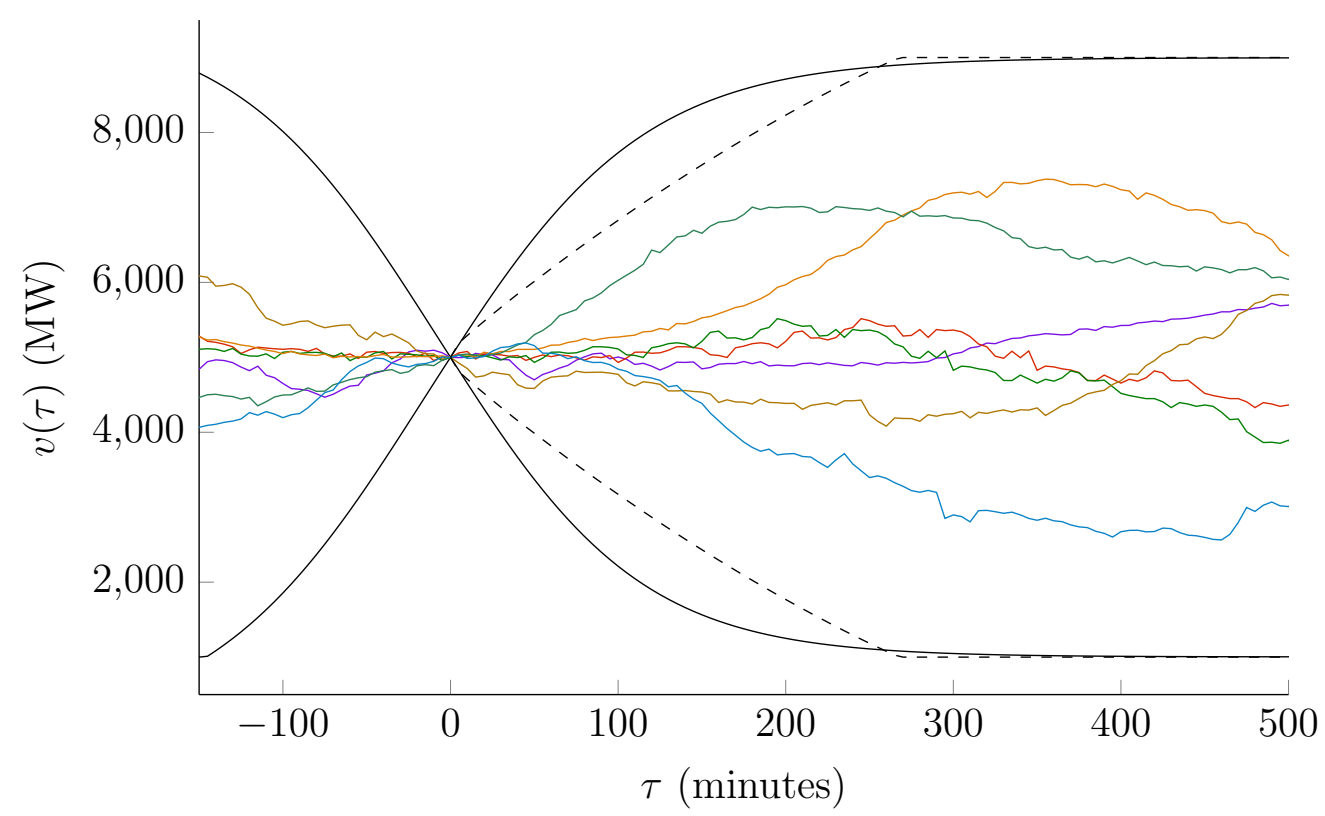

Fig. 5.8 Plot of the flexibility requirement envelope of BPA's net load dataset.

Moreover, the dashed envelope lines in Fig. 5.8 represent the flexibility envelope computed statistically from the net load time series as in Chapter 2. The envelope is also plotted (dashed lines) in the phase-plane of Fig. 5.7. As shown, the statistically averaged envelope from Chapter 2 is less stringent than the dynamical envelope proposed here. It also shows how the ramping rate of the net load is assumed to remain relatively constant on the envelope, since the dashed lines in Fig. 5.7 represent a near constant ramp-rate profile (as seen in Fig. 5.3).

\subsection{Flexibility Adequacy Assessment}

\subsubsection{Flexibility Aggregation}

Let $h(\tau ; k)$ represent the trajectory formed by aggregating power system resources, such that

$$
h(\tau ; k)=\sum_{i} x_{i}(\tau ; k)
$$

All feasible trajectories $h(\tau ; k)$ belong to the set $\mathcal{H}$ of aggregate resources trajectories for a given configuration. There exist two feasible aggregate trajectories $\left\{e_{v}^{\downarrow}(\tau ; k), e_{v}^{\uparrow}(\tau ; k)\right\} \subset \mathcal{H}$ 
constituting the aggregate flexibility envelope enclosing the set $\mathcal{H}$, in line with the envelope enclosure defined in Section 5.3.

\subsubsection{Envelope Enclosure}

Looking at Fig. 5.9, we are interested in evaluating how well the aggregate flexibility envelope of resources, $\left\{e_{h}^{\downarrow}, e_{h}^{\uparrow}\right\}$, encloses the flexibility requirement envelope, $\left\{e_{v}^{\downarrow}, e_{v}^{\uparrow}\right\}$. This is

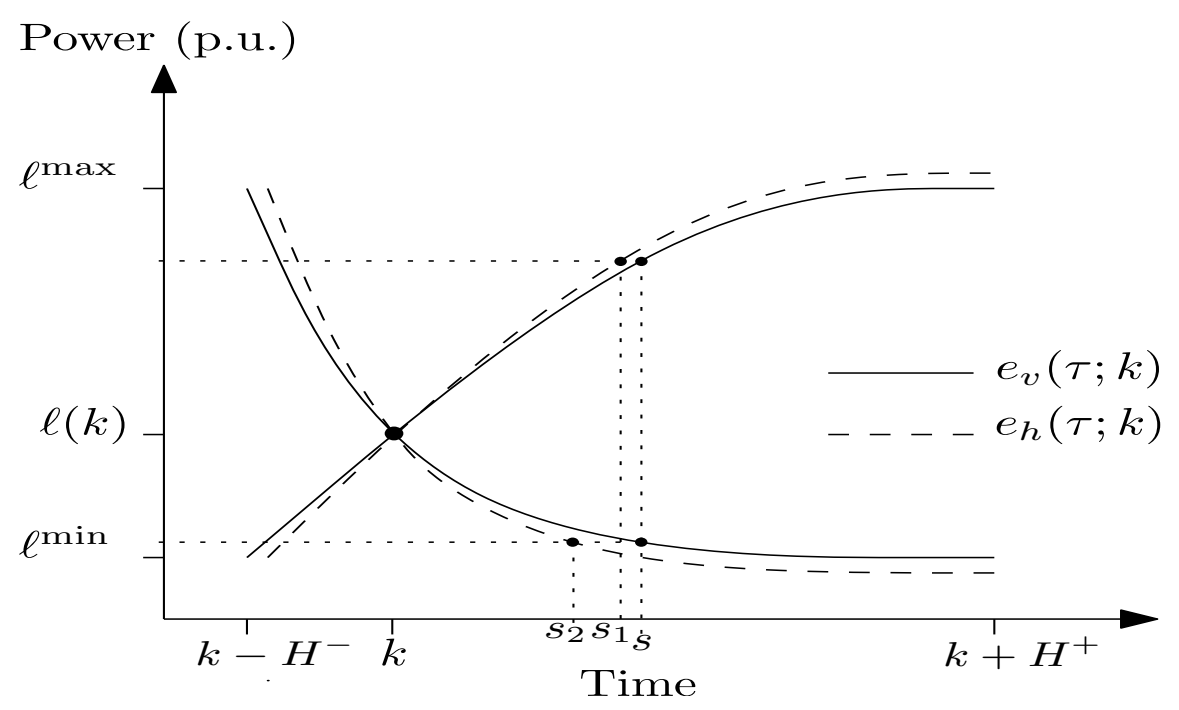

Fig. 5.9 Illustration of flexibility requirement envelope enclosure.

slightly different from envelope enclosure defined in Section 5.3, which seeks to enclose a set of trajectories by an envelope, whereas here we seek to enclose an envelope by another. To begin with, the two envelopes $\left\{e_{h}^{\downarrow}, e_{h}^{\uparrow}\right\}$ and $\left\{e_{v}^{\downarrow}, e_{v}^{\uparrow}\right\}$ must satisfy the initial condition

$$
e_{h}^{\downarrow}(0 ; k)=e_{h}^{\uparrow}(0 ; k)=e_{v}^{\downarrow}(0 ; k)=e_{v}^{\uparrow}(0 ; k)=\ell(k)
$$

which ensures power balance at the forecasted net load level. The envelopes must also satisfy the boundary conditions

$$
\begin{aligned}
e_{h}^{\downarrow}\left(-H^{-} ; k\right) & =e_{h}^{\uparrow}\left(H^{+} ; k\right) \geq \ell^{\max } \\
e_{v}^{\downarrow}\left(-H^{-} ; k\right) & =e_{v}^{\uparrow}\left(H^{+} ; k\right)=\ell^{\max } \\
e_{h}^{\downarrow}\left(H^{+} ; k\right) & =e_{h}^{\uparrow}\left(-H^{-} ; k\right) \leq \ell^{\min } \\
e_{v}^{\downarrow}\left(H^{+} ; k\right) & =e_{v}^{\uparrow}\left(-H^{-} ; k\right)=\ell^{\min }
\end{aligned}
$$


which ensure adequate capacity (i.e. $n=0$ ).

To ensure adequate $n^{\text {th }}$-order ramping capability, enclosing the flexibility requirement envelope by the aggregate flexibility envelope of power system resources entails the $n^{\text {th }}$-order derivatives of the upward aggregate flexibility half-envelope $e_{h}^{\uparrow}(\tau ; k)$ bound from above the $n^{\text {th }}$-order derivatives of the upward flexibility requirement half-envelope $e_{v}^{\uparrow}(\tau ; k)$; see Fig. 5.9. This is done by drawing a horizontal line intersecting both upward half-envelopes at the same output level at $\tau=s$ and $\tau=s_{1}$. The $n^{\text {th }}$-order derivatives evaluated at $\tau=s$ and $\tau=s_{1}$ are such that

$$
\left.\frac{d^{n}}{d \tau^{n}} e_{h}^{\uparrow}(\tau ; k)\right|_{\tau=s_{1}} \geq\left.\frac{d^{n}}{d \tau^{n}} e_{v}^{\uparrow}(\tau ; k)\right|_{\tau=s}
$$

for any $n \geq 1$. This enclosure also entails the $n^{\text {th }}$-order derivatives of the downward aggregate flexibility half-envelope $e_{h}^{\downarrow}(\tau ; k)$ bound from below the $n^{t h}$-order derivatives of the downward flexibility requirement half-envelope $e_{v}^{\downarrow}(\tau ; k)$. Likewise, this is done by drawing a horizontal line intersecting both downward half-envelopes at the same output level at $\tau=s$ and $\tau=s_{2}$. The $n^{\text {th }}$-order derivatives evaluated at $\tau=s$ and $\tau=s_{2}$ are such that

$$
\left.\frac{d^{n}}{d \tau^{n}} e_{h}^{\downarrow}(\tau ; k)\right|_{\tau=s_{2}} \leq\left.\frac{d^{n}}{d \tau^{n}} e_{v}^{\downarrow}(\tau ; k)\right|_{\tau=s}
$$

for any $n \geq 1$.

Again, the comparison is carried out by drawing a horizontal line intersecting the two envelopes at the same output level and comparing the $n^{\text {th }}$-order derivatives at the intersection points. This enables us to seek time-independent evaluation of flexibility adequacy in time-invariant power system planning, in contrast to the traditional time-based evaluation of operating reserve.

\subsubsection{Flexibility Adequacy of Envelope Enclosure}

Now, assuming adequate flexibility already exists in the power system, for every net load realization, $v(\tau ; k) \in \mathcal{V}$, the aggregate response of flexibility resources can generate an equal trajectory, $h(\tau ; k) \in \mathcal{H}$, such that

$$
h(\tau ; k)=v(\tau ; k) \quad \forall \tau \in\left[0, H^{+}\right]
$$


This implies that $\mathcal{V} \subseteq \mathcal{H}$ which, in turn, leads to the aggregate flexibility envelope of resources enclosing the flexibility requirement envelope. This is because the trajectories constituting the flexibility requirement envelope are elements in the set $\mathcal{V}$ which is a subset of the set $\mathcal{H}$.

Conversely, enclosing the flexibility requirement envelope by the aggregate flexibility envelope of resources implies that for every net load realization $v(\tau ; k)$, the aggregate response of resources is capable of generating an equal trajectory $h(\tau ; k)$. This can be achieved by tracking the flexibility requirement envelope during planning, which ensures the aggregate flexibility envelope is at least equal to the flexibility requirement envelope, as per the trackability premise in (5.1)-(5.2). The converse is more interesting from a flexibility planning perspective for guaranteeing adequate system flexibility moving forward.

\subsection{Dynamical Planning with Envelopes}

\subsubsection{Min-Max Approach to Envelope Tracking}

The flexibility requirement envelope consists of two net load realizations which have the maximum deviations (in the $n^{\text {th }}$-order derivatives) but the lowest probabilities of occurring among all other net load realizations enclosed within. In other words, they represent the limit behavior (or limit trajectories) of the net load random process. As per the converse statement stated earlier, tracking the two limit trajectories, while subjecting the resources' trajectories to their flexibility envelopes, is sufficient to ensure envelope enclosure and, consequently, ensure adequate system flexibility.

Dynamically tracking the flexibility requirement envelope constitutes a min-max optimal control problem. From a min-max perspective, we seek "the minimum over the control input of the maximum over the disturbance" [60]. Employing this approach, we want to minimize the cost of tracking the two limit trajectories of the net load random process, while satisfying constraints on the dynamics of the flexibility resources. This ensures robustness against the additive disturbances arising from the net load random process, as long as the maximum over the disturbance (i.e. the envelope) can be tracked. 


\subsubsection{Optimal Envelope Tracking Problem}

We are interested in minimizing a cost functional, subject to envelope enclosure over the horizon $\tau \in\left[-H^{-}, H^{+}\right]$. The present time $k$ occurs at $\tau=0$ which is the point of intersection of the two envelopes and represents the power balance constraint at present time. Essentially, we want to preposition resources for future dispatch in the most economical way, such that they can collectively track the flexibility requirement envelope.

Usually, tracking-control problems involve minimizing the cost associated with a sumof-squares tracking error. Here, we take a different approach that is more suitable for power systems planning. We make use of slack variables to reflect the cost/flexibility trade-off associated with planning resources in the face of inadequate system flexibility. The slack variable at present time $k$ corresponds to load shedding less RES curtailment, whereas everywhere else on $\left[-H^{-}, H^{+}\right]$, the slack variables can be used by the planner to relax the need to strictly track the flexibility requirement envelope. The planner must find the optimal cost/flexibility trade-off depending on the slack variables' constraints and their associated costs. In particular, if they are priced much higher than actual flexibility resources, they should be used as a last resort only. Specific constraints on the envelope's slack variables may come from a cost/benefit [54] or from reliability-based [17] analysis of reserve requirements. Yet, the idea of perfect tracking is raised only rhetorically here to define the notion of flexibility precisely. In real-time operations, there will be imprecise (imperfect) tracking leading to frequency deviations and reflecting certain degree of residual inflexibility in the system, time delays in implementing control decisions, etc.

To simplify the planning problem formulation, the slack variables are considered to be the $(I+1)^{t h}$ flexibility resource, with its own flexibility envelope, where $I$ is the actual number of flexibility resources present in the system. Henceforth, the optimal flexibility envelope tracking problem formulation becomes

$$
\min _{\boldsymbol{x}^{\downarrow}, \boldsymbol{x}^{\uparrow}, \boldsymbol{u}} \int_{-H^{-}}^{H^{+}} \sum_{i=1}^{I+1} C_{i}\left(\boldsymbol{x}_{i}^{\downarrow}(\tau ; k), \boldsymbol{x}_{i}^{\uparrow}(\tau ; k), u_{i}\right) d \tau
$$

subject to: 
Power Balance (Initial Conditions) at $k(\tau=0)$

$$
\begin{aligned}
& \sum_{i=1}^{I+1} u_{i} \cdot x_{i}^{\uparrow}(0 ; k)=e_{v}^{\uparrow}(0 ; k)=\ell(k) \\
& \sum_{i=1}^{I+1} u_{i} \cdot x_{i}^{\downarrow}(0 ; k)=e_{v}^{\downarrow}(0 ; k)=\ell(k)
\end{aligned}
$$

Envelope Tracking $\forall \tau \in\left[-H^{-}, H^{+}\right]$

$$
\begin{aligned}
& \sum_{i=1}^{I+1} u_{i} \cdot x_{i}^{\downarrow}(\tau ; k)=e_{v}^{\downarrow}(\tau ; k) \\
& \sum_{i=1}^{I+1} u_{i} \cdot x_{i}^{\uparrow}(\tau ; k)=e_{v}^{\uparrow}(\tau ; k)
\end{aligned}
$$

Flexibility Constraints $\forall \tau \in\left[-H^{-}, H^{+}\right], i=1, \ldots, I+1$, and $n=0, \ldots, N$

$$
\begin{aligned}
\frac{d^{n}}{d \tau^{n}} x_{i}^{\downarrow}(\tau ; k) & \geq u_{i} \cdot \frac{d^{n}}{d \tau^{n}} e_{x, i}^{\downarrow}(\tau ; k) \\
\frac{d^{n}}{d \tau^{n}} x_{i}^{\uparrow}(\tau ; k) & \leq u_{i} \cdot \frac{d^{n}}{d \tau^{n}} e_{x, i}^{\uparrow}(\tau ; k)
\end{aligned}
$$

Boundary Conditions $\forall i=1, \ldots, I+1$

$$
\begin{array}{r}
x_{i}^{\uparrow}\left(-H^{-} ; k\right)=x_{i}^{\downarrow}\left(H^{+} ; k\right) \\
x_{i}^{\downarrow}\left(-H^{-} ; k\right)=x_{i}^{\uparrow}\left(H^{+} ; k\right)
\end{array}
$$

where $u_{i}$ is the binary commitment variable (on/off) of resource $i$, and the state vectors, $\boldsymbol{x}_{i}^{\downarrow}(\tau ; k), \boldsymbol{x}_{i}^{\uparrow}(\tau ; k)$, in $(5.27)$

$$
\begin{aligned}
& \boldsymbol{x}_{i}^{\downarrow}(\tau ; k)=\left[x_{i}^{\downarrow}(\tau ; k), \frac{d}{d \tau} x_{i}^{\downarrow}(\tau ; k), \ldots, \frac{d^{N}}{d \tau^{N}} x_{i}^{\downarrow}(\tau ; k)\right]^{T} \\
& \boldsymbol{x}_{i}^{\uparrow}(\tau ; k)=\left[x_{i}^{\uparrow}(\tau ; k), \frac{d}{d \tau} x_{i}^{\uparrow}(\tau ; k), \ldots, \frac{d^{N}}{d \tau^{N}} x_{i}^{\uparrow}(\tau ; k)\right]^{T}
\end{aligned}
$$

can be penalized to reflect the cost of flexibility as quantified by the $n^{t h}$-order derivatives.

It is worth noting that in some markets flexibility has already been priced in the form 
of first-order ramping products [69]. This begs the question whether, someday, we are going to package the higher $n^{\text {th }}$-order derivatives as flexibility products. After all, the first-order ramping is the first derivative from a capacity (as in megawatts) market perspective, whereas it is the second derivative from an energy (as in megawatt-hours) market perspective.

\subsection{Application Example}

The following example illustrates operational planning with dynamical flexibility envelopes. The premise is that individual resources must be prepositioned appropriately ahead of time, such that their total scheduled output meets the power balance constraint, while their aggregate flexibility envelope encloses the flexibility requirement envelope over the operational horizon. Envelope enclosure over the forward horizon assesses flexibility deployment, whereas enclosure over the backward horizon assesses flexibility recovery, as discussed earlier. Two flexibility resource portfolios will be compared: one portfolio contains resources having a dynamic ramping profile, whereas the other contains resources with a constant maximum ramp profile. The unit commitment problem is first solved, followed by simulated economic dispatch to benchmark the operating performance of both portfolios. We will show the former portfolio with dynamic ramping resources to be more flexible and cost-effective.

\subsubsection{Problem Setup}

Net Load Model: The net load is modeled as a first-order linear time-invariant (LTI) system. Let $v(\tau ; k)$ be the net load output, such that

$$
\tau_{v} \frac{d}{d \tau} v(\tau ; k)+v(\tau ; k)=f_{v}(\tau)
$$

where $\tau_{v}=15$ minutes is the system's net load time constant, $\tau$ is the horizon time variable, $k$ is the decision-making period, and $f_{v}$ is a normally-distributed random input with $\mu=100 \mathrm{MW}$ and $\sigma=50 \mathrm{MW}$. Fig. 5.10 shows the phase plane region of the net load, after simulating (5.38) for 1000005 -minute periods, starting at $v(0 ; k)=100 \mathrm{MW}$.

To plot the upward segment, $e_{v}^{\uparrow}(\tau ; k)$, and the downward segment, $e_{v}^{\downarrow}(\tau ; k)$, of the flexibility requirement envelope, we set $f(\tau)=f_{v}^{\max }$ and $f(\tau)=f_{v}^{\min }, \forall \tau$, respectively. Fig. 5.10 


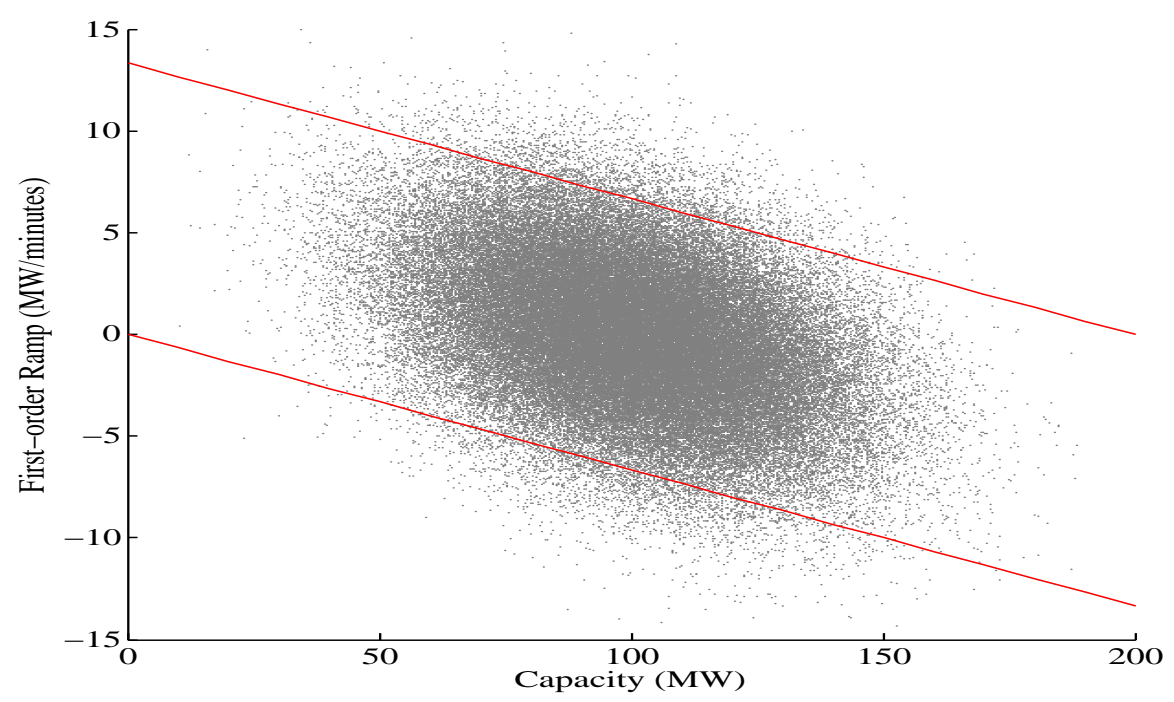

Fig. 5.10 Phase-plane characteristics of the net load. Solid lines represent the flexibility requirement envelope in the phase plane.

shows also plots the phase-plane trajectories of the flexibility requirement envelope, where $f^{\text {min }}$ and $f^{\max }$ are taken to be the $95^{\text {th }}$ percentiles of $f_{v}$ (i.e., $\mu \pm 2 \sigma=100 \pm 100 \mathrm{MW}$ ). Fig. 5.11 plots the flexibility requirement envelope in the time domain, as well as sample net load trajectories for $v(0 ; \tau)=100 \mathrm{MW}$.

Portfolio 1: Dynamic Ramp Profile: Consider ten identical resources modeled as first-order LTI systems. Let $x_{i}(\tau ; k)$ be the power output of resource $i$, such that

$$
\tau_{x, i} \frac{d}{d \tau} x_{i}(\tau ; k)+x_{i}(\tau ; k)=f_{x, i}(\tau)
$$

where $P_{i}^{\min } \leq f_{x, i}(\tau) \leq P_{i}^{\max }(\mathrm{MW})$ is the decision input, and $\tau_{x, i}$ minutes is the time constant of resource $i$. Hence, the resources' maximum ramp rates are dynamic and depend on the power output level. Table 5.2 shows the output characteristics and operational costs of the resources, where $a_{i}$ is their no-load cost and $b_{i}$ is their incremental cost.

Table 5.2 Operational Characteristics and Costs of Resources-Portfolio 1

\begin{tabular}{cccccc}
\hline & $\begin{array}{c}\tau_{x, i} \\
\text { (minutes) }\end{array}$ & $\begin{array}{c}P_{i}^{\min } \\
(\mathrm{MW})\end{array}$ & $\begin{array}{c}P_{i}^{\max } \\
(\mathrm{MW})\end{array}$ & $\begin{array}{c}a_{i} \\
(\$)\end{array}$ & $\begin{array}{c}b_{i} \\
(\$ / \mathrm{MW})\end{array}$ \\
\hline$i$ & 15 & 0 & 30 & 200 & $i$ \\
\hline
\end{tabular}




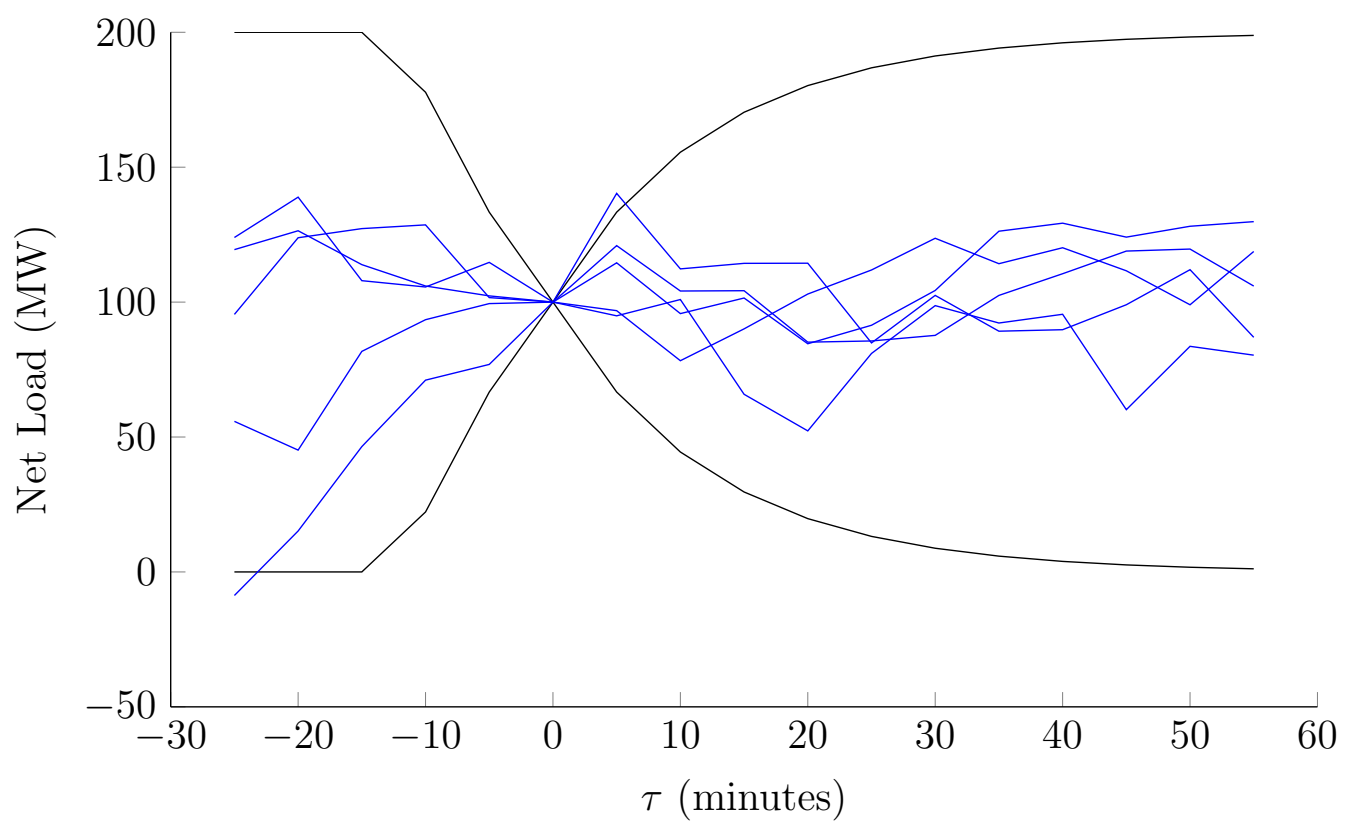

Fig. 5.11 Time-domain plot of the flexibility requirement envelope and some sample net load random process realizations.

Portfolio 2: Linear Ramp Profile: Consider now ten identical resources having a constant maximum ramp rate (independent of the resource current power output)

$$
R_{i}^{\max }=\frac{P_{i}^{\max }}{2 \tau_{x, i}}=1(\mathrm{MW} / \text { minute })
$$

where $P_{i}^{\max }$ and $\tau_{x, i}$ were defined in the previous subsection. Table 5.3 shows the output characteristics and operational costs of the resources. In other words, these are the same resources as in portfolio 1 but given a constant maximum ramp rate $R_{i}^{\max }$, rather than being dynamic as a function of the power output level.

Table 5.3 Operational Characteristics and Costs of Resources-Portfolio 2

\begin{tabular}{cccccc}
\hline & $\begin{array}{c}R_{i}^{\max } \\
(\mathrm{MW} / \text { minute })\end{array}$ & $\begin{array}{c}P_{i}^{\min } \\
(\mathrm{MW})\end{array}$ & $\begin{array}{c}P_{i}^{\max } \\
(\mathrm{MW})\end{array}$ & $\begin{array}{c}a_{i} \\
(\$)\end{array}$ & $\begin{array}{c}b_{i} \\
(\$ / \mathrm{MW})\end{array}$ \\
\hline$i$ & 1 & 0 & 30 & 200 & $i$ \\
\hline
\end{tabular}




\subsubsection{Unit Commitment Problem}

Unit commitment is performed for a single hourly period for the two resource portfolios described above. The horizon $\tau \in[-60,60]$ minutes is discretized every $T=5$ minutes. The problem, a mixed-integer linear program, is formulated in according to the min-max philosophy laid out in Section 5.6. It is worth underlining that the unit commitment formulation, described next, is novel in the sense that it models flexibility resources' ramping dynamically using linear constraints only. In current practices, dynamical ramping is thought to pose a challenge, due to potentially causing non-trivial nonlinearities in the unit commitment formulation. There have been limited studies to tackle this issue - see [70] for example. The key to the simplicity of our approach is a by-product of the use of envelopes to model dynamic ramping processes.

The mixed-integer linear programming formulation for the flexibility envelope constrained unit commitment problem consists of:

$$
\begin{aligned}
& \min \left(\sum_{i=1}^{10} a_{i} u_{i}+b_{i} x_{i}(0)\right)+c_{s}\left(S^{\uparrow}+S^{\downarrow}\right) \\
& +\sum_{m=1}^{12}\left(\sum_{i=1}^{10} b_{i} x_{i}^{\downarrow}(-m)+c_{s} e_{S}^{\downarrow}(-m)\right) \\
& +\sum_{m=1}^{12}\left(\sum_{i=1}^{10} b_{i} x_{i}^{\uparrow}(-m)+c_{s} e_{S}^{\uparrow}(-m)\right) \\
& +\sum_{m=1}^{12}\left(\sum_{i=1}^{10} b_{i} x_{i}^{\downarrow}(m)+c_{s} e_{S}^{\downarrow}(m)\right) \\
& +\sum_{m=1}^{12}\left(\sum_{i=1}^{10} b_{i} x_{i}^{\uparrow}(m)+c_{s} e_{S}^{\uparrow}(m)\right)
\end{aligned}
$$

Here, the objective is to minimize the unit commitment cost for the single hourly period. The cost function comprises of a penalty term associated with the resources' commitment status and the actual scheduled output levels of the resources at $\tau=0$ in (5.41). The potential cost of flexibility deployment of the resources over $\tau \in[-60,0) \cup(0,60]$ minutes is handled in (5.42)-(5.45). The terms $S^{\uparrow}, S^{\downarrow}, e_{S}^{\uparrow}$, and $e_{S}^{\downarrow}$ are infinitely-flexible resources (i.e., slack variables), to help balance the cost/benefit of planning flexibility, as well as insuring feasibility in cases where flexibility is scarce. They are priced at a much higher rate than 
the other resources, $c_{s}=\$ 1000 / \mathrm{MW}$, and should be used as last resort.

This is subject to

$$
\begin{aligned}
\sum_{i=1}^{10} x_{i}(0)+S^{\uparrow}-S^{\downarrow} & =\ell(0) \\
S^{\uparrow}, S^{\downarrow} & \geq 0
\end{aligned}
$$

where (5.46) and (5.47) ensure the power balance for the hourly net load forecast level $\ell(0)$. Constraints (5.48) and (5.50) ensure tracking of the flexibility requirement envelope backward in time, whereas constraints (5.49) and (5.51) ensure tracking of the flexibility requirement envelope forward in time.

$$
\begin{array}{ll}
\sum_{i=1}^{10} x_{i}^{\downarrow}(-m)=e_{v}^{\downarrow}(-m)+e_{S}^{\downarrow}(-m) ; & m=1, \ldots, 12 \\
\sum_{i=1}^{10} x_{i}^{\downarrow}(m)=e_{v}^{\downarrow}(m)+e_{S}^{\downarrow}(m) ; & m=1, \ldots, 12 \\
\sum_{i=1}^{10} x_{i}^{\uparrow}(-m)=e_{v}^{\uparrow}(-m)+e_{S}^{\uparrow}(-m) ; & m=1, \ldots, 12 \\
\sum_{i=1}^{10} x_{i}^{\uparrow}(m)=e_{v}^{\uparrow}(m)+e_{S}^{\uparrow}(m) ; & m=1, \ldots, 12
\end{array}
$$

The right-hand sides of (5.48)-(5.51) are determined by (5.52)-(5.56). These represent the upward and downward evolution of the flexibility requirement envelope, both forward and backward in time, starting from $\ell(0)$ and through each sub-hourly time interval $m=$ $1, \ldots, 12$. Note here that none of these are optimization variables; they only parametrize 
the flexibility requirement envelope.

$$
\begin{aligned}
& e_{v}^{\uparrow}(m)=\min \left\{200, e_{v}^{\uparrow}(m-1)+T \frac{200-e_{v}^{\uparrow}(m-1)}{\tau_{v}}\right\} \\
& e_{v}^{\downarrow}(m)=\max \left\{0, e_{v}^{\downarrow}(m-1)-T \frac{e_{v}^{\uparrow}(m-1)}{\tau_{v}}\right\} \\
& e_{v}^{\uparrow}(-m)=\max \left\{0, e_{v}^{\uparrow}(-(m-1))-T \frac{200-e_{v}^{\uparrow}(-m)}{\tau_{v}}\right\} \\
& e_{v}^{\downarrow}(-m)=\min \left\{200, e_{v}^{\downarrow}(-(m-1))+T \frac{e_{v}^{\uparrow}(-m)}{\tau_{v}}\right\} \\
& e_{v}^{\uparrow}(0)=e_{v}^{\downarrow}(0)=\ell(0)
\end{aligned}
$$

Next, constraints (5.57) and (5.58) limit the available flexible capacity, to ensure that the forward- and backward-in-time envelopes reach the same minimum and maximum capacities. Along with constraints (5.62), (5.66), (5.70), and (5.74), they represent envelope boundary conditions for each resource $i$

$$
\begin{array}{rlrl}
P_{i}^{\min } u_{i} & \leq x_{i}^{\min } \leq x_{i}(0) ; & & i=1, \ldots, 10 \\
x_{i}(0) & \leq x_{i}^{\max } \leq P_{i}^{\max } u_{i} ; & i=1, \ldots, 10
\end{array}
$$

Finally, to perform unit commitment with Portfolio 1, which has dynamic ramping rate limitations, the following constraints are used. Constraints (5.59)-(5.61) and (5.63)-(5.65) bound the backward-in-time potential flexibility deployment of a resource by its flexibility envelope, whereas constraints (5.67)-(5.69) and (5.71)-(5.73) bound the forward-in-time potential flexibility deployment of a resource by its flexibility envelope.

Up and Backward for $i=1, \ldots, 10$

$$
\begin{array}{ll}
x_{i}^{\downarrow}(-1) \geq x_{i}(0) & \\
x_{i}^{\downarrow}(-(m+1)) \geq x_{i}^{\downarrow}(-m) ; & m=1, \ldots, 11 \\
x_{i}^{\downarrow}(-(m+1))-x_{i}^{\downarrow}(-m) \leq T \frac{x_{i}^{\downarrow}(-(m+1))-P_{i}^{\min }}{\tau_{x, i}} ; & m=0, \ldots, 11 \\
x_{i}^{\downarrow}(-12)=x_{i}^{\max } &
\end{array}
$$


Down and Backward $i=1, \ldots, 10$

$$
\begin{array}{ll}
x_{i}^{\uparrow}(-1) \leq x_{i}(0) & \\
x_{i}^{\uparrow}(-(m+1)) \leq x_{i}^{\uparrow}(-m) ; & m=1, \ldots, 11 \\
x_{i}^{\uparrow}(-m)-x_{i}^{\uparrow}(-(m+1)) \leq T \frac{P_{i}^{\max }-x_{i}^{\uparrow}(-(m+1))}{\tau_{x, i}} ; & m=0, \ldots, 11 \\
x_{i}^{\uparrow}(-12)=x_{i}^{\min } &
\end{array}
$$

Down and Forward $i=1, \ldots, 10$

$$
\begin{array}{ll}
x_{i}^{\downarrow}(1) \leq x_{i}(0) & \\
x_{i}^{\downarrow}(m+1) \leq x_{i}^{\downarrow}(m) ; & m=1, \ldots, 11 \\
x_{i}^{\downarrow}(m)-x_{i}^{\downarrow}(m+1) \leq T \frac{x_{i}^{\downarrow}(m)-P_{i}^{\min }}{\tau_{x, i}} ; & m=0, \ldots, 11 \\
x_{i}^{\downarrow}(12)=x_{i}^{\min } &
\end{array}
$$

Up and Forward $i=1, \ldots, 10$

$$
\begin{array}{ll}
x_{i}^{\uparrow}(1) \geq x_{i}(0) & \\
x_{i}^{\uparrow}(m+1) \geq x_{i}^{\uparrow}(m) & m=1, \ldots, 11 \\
x_{i}^{\uparrow}(m+1)-x_{i}^{\uparrow}(m) \leq T \frac{P_{i}^{\max }-x_{i}^{\uparrow}(m)}{\tau_{x, i}} & m=0, \ldots, 11 \\
x_{i}^{\uparrow}(12)=x_{i}^{\max } &
\end{array}
$$

We note that the dynamic maximum ramping limitations are found here in (5.61), (5.65), (5.69), and (5.73).

Similarly, to perform unit commitment with Portfolio 2, which has constant maximum ramping rate limitations, the same constraints as in (5.59)-(5.74) are used, with the exception that the ramp rate limitations in (5.61), (5.65), (5.69), and (5.73) respectively, which 
are replaced with

$$
\begin{aligned}
x_{i}^{\downarrow}(-(m+1))-x_{i}^{\downarrow}(-m) & \leq T \cdot R_{i}^{\max } \\
x_{i}^{\uparrow}(-m)-x_{i}^{\uparrow}(-(m+1)) & \leq T \cdot R_{i}^{\max } \\
x_{i}^{\downarrow}(m)-x_{i}^{\downarrow}(m+1) & \leq T \cdot R_{i}^{\max } \\
x_{i}^{\uparrow}(m+1)-x_{i}^{\uparrow}(m) & \leq T \cdot R_{i}^{\max }
\end{aligned}
$$

for all $i=1, \ldots, 10$ and $m=0, \ldots, 11$.

\subsubsection{Unit Commitment Results and Discussion}

The mathematical program is solved repeatedly for a range of $\ell(0)$ values, to compare the unit commitment results of the two power system resource portfolios. Fig. 5.12 shows the unit commitment results of both portfolios. The first subplot compares the scheduling costs without the no-load costs, whereas the second subplot compares the scheduling costs with the no-load costs. Together, they illustrate Portfolio 1 to be more cost-effective than Portfolio 2. The third subplot compares the number of committed resources, where Portfolio 1 resulted in fewer resources committed in comparison to Portfolio 2. The fourth subplot shows usage of slack variables to help with balancing the net load forecast in constraint (5.46). Portfolio 1 is capable of meeting the net load forecast without using the slack variables, whereas Portfolio 2 had to resort to using the slack variables for certain net load forecast levels. These slack variable uses reflect the need to deploy load shedding $\left(S^{\uparrow}\right)$ or renewable energy curtailment $\left(S^{\downarrow}\right)$, in the case of insufficient supply of flexibility by the committed resources. Finally, the fifth subplot shows slack deployment over the operational horizon to help with meeting (tracking) the flexibility requirement envelope in constraints (5.48)-(5.51). This reflects potential future needs to deploy load shedding or renewable energy curtailment in real-time operation. Portfolio 1 was better at reducing the usage of these slack variables, compared to Portfolio 2. Overall, the results in Fig. 5.12 indicate that the dynamic ramp profile (i.e., in Portfolio 1) is more flexible than the constant maximum ramp profile (i.e., in Portfolio 2) with reductions in scheduling costs, load shedding, and renewable energy curtailment. As discussed earlier, the constant maximum ramp profile may further increase the long-run operational costs, due to the costs associated with rotor fatigue [66]. 

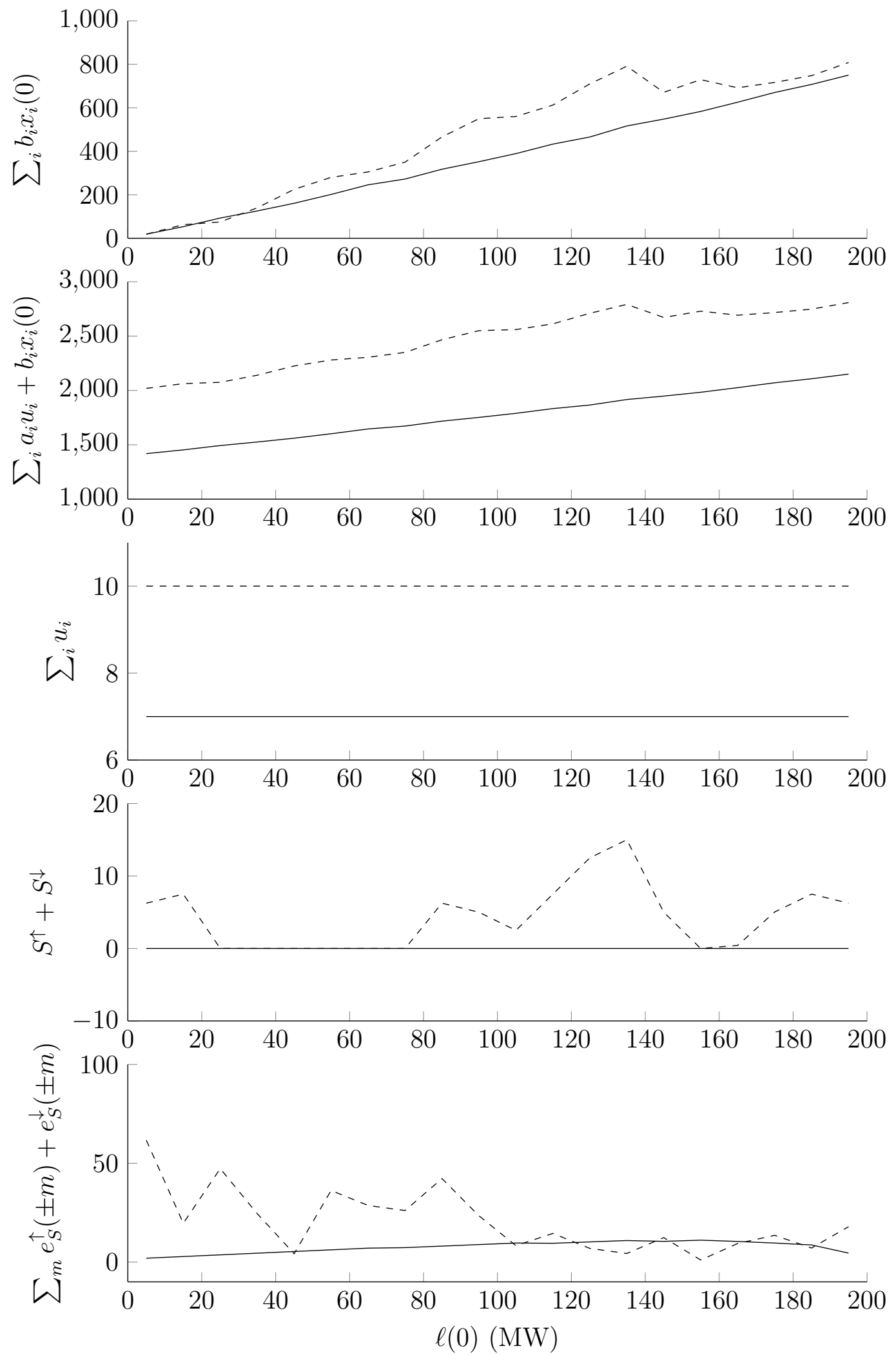

Fig. 5.12 Unit commitment solved repeatedly for a range of $\ell(0)$ values. Results for Portfolio 1 (solid). Results for Portfolio 2 (dashed). 


\subsubsection{Economic Dispatch Problem}

The goal next is to compare the performance of the two portfolios in real-time operations. Therefore, we fix the forecast $\ell(k)$ to $100 \mathrm{MW}$ and perform unit commitment to obtain resources' commitment for this particular forecast level. Then, a receding-horizon economic dispatch is performed assuming the fixed unit commitment schedule to assess how the prior unit commitment decisions can lead to better or worse outcomes in real-time dispatch operations.

The unit commitment is carried out for the same hourly forecast of $\ell(0)=100 \mathrm{MW}$ as in the previous sub-section, and generation dispatch is carried out, neglecting the no-load costs, every 5 minutes of the hour. At every sub-hourly time step the realization of the randomly-varying net load is observed, and generation set points are updated ensuring both the satisfaction of the power balance at the current time and envelope enclosure going forward.

Specifically, the net load is initialized with $\ell(k)=100 \mathrm{MW}$ and $J$ random trajectories of the net load are generated by randomizing $f_{v}$ every 5 minutes for 12 sub-hourly time steps. $f_{v}$ is randomized according to the Gaussian distribution, as described earlier. Each net load realization and its corresponding dispatch decisions represent how the system would have been run in that hour.

To assess the quality of flexibility provided by each portfolio, we compute the expected energy not served $\left(\mathrm{EENS}_{J}\right)$ and the expected energy curtailed $\left(\mathrm{EEC}_{J}\right)$ from all $J$ realizations of the net-load random process:

$$
\begin{aligned}
\operatorname{EENS}_{J} & =\frac{1}{J} \frac{T}{60} \sum_{j=1}^{J} \sum_{m=1}^{12} S_{j}^{\uparrow}(m) \\
\operatorname{EEC}_{J} & =\frac{1}{J} \frac{T}{60} \sum_{j=1}^{J} \sum_{m=1}^{12} S_{j}^{\downarrow}(m)
\end{aligned}
$$

where $S_{j}^{\downarrow}(m)$ and $S_{j}^{\uparrow}(m)$ correspond to renewable power curtailment and load shedding, respectively, of the economic dispatch decisions at time steps $m=1, \ldots, 12$ for net-load realizations $j=1, \ldots, J$. The number of realizations $J$ must be large enough for EENS and EEC to converge reasonably well. 


\subsubsection{Economic Dispatch Results and Discussion}

Reasonable convergence was achieved within $J=500$ simulation runs. Table 5.4 shows the converged EENS and EENS values, as well as the total expected dispatch cost over one hour of operations, ECost. Comparing results, it is evident that Portfolio 1-dispatching

Table 5.4 Expected Energy Not Served (EENS), Expected Energy Curtailed (EEC), and Total Expected Cost (EC)

\begin{tabular}{lcc}
\hline & Portfolio 1 & Portfolio 2 \\
\hline EENS (MWh) & 0.1110 & 0.0348 \\
EEC (MWh) & 0.1282 & 5.3570 \\
EC $(\$)$ & 4513 & 6500 \\
\hline
\end{tabular}

seven committed resources only - is more flexible and cost-effective than Portfolio 2 - which is dispatching ten committed resources. The only case where Portfolio 2 outperforms Portfolio 1 is when comparing EENS (about $69 \%$ more), but this was achieved at a much higher expected cost (EC) (about 30\% more). Therefore, the evidence is such that the consideration of dynamically-varying maximum ramping by power system resources is advantageous. Nonetheless, fine-tuning the flexibility/cost trade-off via the dispatch and the envelope slack variables is necessary for the decision-maker to obtain acceptable performance. For example, performance with respect to EENS for Portfolio 1 could be improved by increasing the penalty for load shedding; obviously, this would come at an increase in expected cost.

\subsection{Summary}

In this chapter, we attempted to modernize and extend the concept of power system flexibility beyond the traditional time-based operating reserve paradigm. In contrast to other literature on flexibility planning, which rely on capacity and average ramping assumptions, the methodology in this chapter is precise and is motivated by the notion of trajectory tracking and its relevance to $n^{\text {th }}$-order dynamics. We used dynamical envelopes to bound all feasible trajectories of resources as well as all plausible realizations of the net load. The envelopes defined operating reserve dynamics and are thought to replace traditional reserve 
types. Furthermore, we also asserted that the aggregate flexibility envelope of resources must enclose the flexibility requirement envelope arising from net load, to ensure adequate system flexibility. Flexibility planning with envelopes was also formulated and illustrated by unit commitment and receding-horizon economic dispatch examples, which showed how the trajectorial approach proposed in this chapter is more cost effective than others assuming constant maximum ramping. For all these reasons, we argue strongly in favor of reexamining how operating reserves are defined and scheduled. This chapter has offered a solid base to initiate this reflection across the industry. 


\section{Chapter 6}

\section{Conclusion}

\subsection{Dissertation Overview}

This dissertation addressed operational challenges associated with the increasing penetration of renewable energy sources in power systems. The main challenges are increased variability and uncertainty beyond what current practices are capable of managing economically. Therefore, we proposed the concept of flexibility envelopes to fully capture this variability and uncertainty during planning. The new concept required that resources are to be scheduled, such that their aggregate flexibility envelope encloses the flexibility requirement envelope arising from the net load variability and uncertainty, while also meeting the net load forecast level.

Chapter 1 reviewed the state of the art on current operational planning practices. The main conclusion was that current practices were based on the traditional operating reserve paradigm which unsatisfactorily captured the net load variability and uncertainty characteristics, whereas the frequency content of the net load periodogram indicated that those characteristics spanned multiple timescales.

Chapter 2 provided a systematic way of defining and calculating the flexibility requirement envelope, by statistically analyzing empirical net load data. Chapter 2 also provided a systematic way of defining and calculating the flexibility envelope of a power system resource. Planning with flexibility envelopes was formulated as a linear program, whereby the resources had to be scheduled, such that their projected aggregate flexibility envelope enclosed the project flexibility requirement envelope, to guarantee adequate system flexibility. The example provided illustrated the effectiveness of the envelopes concept 
at capturing the net load variability and uncertainty, as well as improving the system's flexibility performance.

One important implication of the contrast between traditional reserve types and the proposed flexibility envelope is that traditional reserve definitions are becoming gradually obsolete and incapable of representing operating reserve requirements in power systems admitting high levels of renewable energy sources. On the other hand, the flexibility requirement envelope is able to capture such characteristics more effectively. Therefore, we argue in favor of replacing these definitions by the proposed envelopes concept, which also brings unity among operational practices across the power industry. This is also in line with ramping products being recently introduced in several energy markets, since the envelope's shape exhibits a ramping profile and can be procured through such ramping products.

Chapter 3 expanded on the envelopes concept proposed in Chapter 2, to include energy flexibility. The flexibility envelopes were re-modeled in terms of energy capacity and ramping, rather than power capacity and ramping only. This redefined the notion of operating reserve from an energy-centric perspective, which is novel to the state of the art and current operating reserve practices. The energy-centric paradigm is more suitable for optimizing power systems with energy storage, demand response, and hydrothermal generation resources. The effectiveness of the energy-centric paradigm was illustrated by a planning example of a power system with energy storage resources.

Chapter 4 proposed a new stochastic approach that circumvents the challenges of scenario-based stochastic planning, whose two main disadvantages are the exorbitant computational costs arising from the curse of dimensionality and the computational complexity of scenario construction and reduction techniques. In this chapter, we specifically proposed probabilistic envelope enclosure, by calculating probability-weighted flexibility requirement envelopes, where the system's resources are required to enclose these envelopes. We showed, via a planning example, the potential benefits of such approach by reducing the total dispatch cost and improving system technical performance. If one thinks of the probabilityweighted flexibility requirement envelopes as a form of scenario reduction, then those envelopes and their corresponding probability weights are very easy to calculate statistically from net load empirical data. Thus, the proposed probabilistic envelopes approach is much more computationally tractable than stochastic planning.

Chapter 5 made a substantial but much needed leap in power system planning practices, thus opening new research avenues for the state of the art to keep pushing forward. 
It argued for the need to move beyond the current static planning practices based on capacity and average ramping considerations. This is because sustainable power systems are expected to become more dynamical, with the increased penetration of renewable energy, demand response, energy storage, and flexible generation. Therefore, Chapter 5 proposed a dynamical optimization approach via the flexibility envelopes, to represent higher-order dynamics of the power balance between resources and the net load. Consequently, the traditional reserve paradigm was replaced by the notion of dynamical operating reserve, exhibiting higher order dynamics. Furthermore, one implication, market-wise, of the work in Chapter 5 is generalizing the aforementioned ramping products to higher order dynamics. For example, it argues for the possibility of introducing acceleration products in the electricity market.

\subsection{Recommendations for Future Work}

Several recommendation for future work have already been mentioned throughout the dissertation. Here, we re-articulate those recommendation, along with several others, that could potentially contribute to advancing the state of the art further.

1. Incorporate transmission constraints into planning with flexibility envelopes. This would require modeling nodal envelopes, at each node (bus) in the power grid. In this dissertation, the flexibility envelopes of individual resources were lumped together, without taking into account the interconnections between nodes. Moreover, the net load was treated as coming from a single bus in this dissertation, thus giving rise to a single flexibility requirement envelope.

2. Formulate unit commitment and longer-term planning with the proposed envelopes concept. The planning examples provided focused on receding-horizon economic dispatch. On a longer-term planning scale, it would be interesting to see how the flexibility envelopes approach can be adapted to nontrivial constraints relating to generation reliability and long term investment decisions.

3. Conduct a detailed integration study on a realistic power system admitting high penetration level of renewable energy sources, to benchmark the effectiveness of the proposed approaches in real life situations. Besides time limitations, a detailed and 
practical integration study would have been less effective at illustrating the conceptual developments made in this dissertation. Thus, we opted for simplified examples, to help illustrate the many novel concepts interlinked in this dissertation.

4. Investigate the effectiveness of the probabilistic envelopes approach, in comparison to stochastic planning. Chapter 4 provided benchmark results for the probabilistic envelopes approach, with comparison to the deterministic envelops approach from Chapter 1, and without comparing its performance to stochastic optimization. This is an ongoing research task at the moment.

5. Apply the energy-centric approach and the dynamical approach to microgrid optimization, where energy can be limited and resources can behave more dynamically.

6. Apply the energy-centric approach to multi-carrier energy scenarios, such as the cooptimization of the power grid and the natural gas system.

7. Investigate the impact of higher-order dynamics on a power system's security, instead of just capacity and average ramping.

8. Model the higher-order dynamics of the net load variability and uncertainty for wind and solar penetration. 


\section{References}

[1] M. Milligan, E. Ela, J. Hein, T. Schneider, G. Brinkman, and P. Denholm, "Bulk electric power systems: Operations and transmission planning," tech. rep., NREL, Golden, CO, 2012.

[2] E. Ela and B. Kirby, "ERCOT event on february 26, 2008: Lessons learned," tech. rep., NREL, Golden, CO, 2008.

[3] F. Bouffard and M. Ortega-Vazquez, "The value of operational flexibility in power systems with significant wind power generation," in Proc. 2011 IEEE Power and Energy Society General Meeting, (Detroit, MI), pp. 1-5.

[4] North American Electric Reliability Corporation, "Accommodating high levels of variable generation," tech. rep., NERC, Princeton, NJ, 2009.

[5] International Energy Agency, "Harnessing variable renewables: A guide to the balancing challenge," tech. rep., OECD/IEA, Paris, France, 2011.

[6] E. Ela, M. Milligan, B. Parsons, D. Lew, and D. Corbus, "The evolution of wind power integration studies: Past, present, and future," in Proc. 2009 IEEE Power Energy Society General Meeting, (Calgary, AB), pp. 1-8.

[7] E. Lannoye, D. Flynn, and M. O'Malley, "The role of power system flexibility in generation planning," in Proc. 2011 IEEE Power and Energy Society General Meeting, (Detroit, MI), pp. 1-6.

[8] M. Carrión and J. Arroyo, "A computationally efficient mixed-integer linear formulation for the thermal unit commitment problem," IEEE Trans. Power Syst., vol. 21, pp. 1371-1378, Aug 2006.

[9] J. Kiviluoma, P. Meibom, A. Tuohy, N. Troy, M. Milligan, B. Lange, M. Gibescu, and M. O'Malley, "Short-term energy balancing with increasing levels of wind energy," IEEE Trans. Sustain. Energy, vol. 3, pp. 769-776, Oct. 2012. 
[10] F. Bouffard and F. D. Galiana, "Stochastic security for operations planning with significant wind power generation," IEEE Trans. Power Syst., vol. 23, pp. 306-316, May 2008.

[11] D. Chen and M. York, "Predictive economic dispatch with time," in Proc. 2011 IEEE Power and Energy Society General Meeting, (Detroit, MI), pp. 1-8.

[12] S. Mei, Y. Wang, and Z. Sun, "Robust economic dispatch considering renewable generation," in Proc. 2011 IEEE PES Innovative Smart Grid Technologies Asia, (Perth, WA), pp. 1-5.

[13] Y. G. Rebours, D. S. Kirschen, M. Trotignon, and S. Rossignol, "A survey of frequency and voltage control ancillary services - Part I: Technical features," IEEE Trans. Power Syst., vol. 22, pp. 350-357, Feb. 2007.

[14] S. T. Lee and Z. A. Yamayee, "Load-following and spinning-reserve penalties for intermittent generation," IEEE Trans. Power App. Syst., vol. PAS-100, pp. 1203-1211, Mar. 1981.

[15] L. Xie, P. M. S. Carvalho, L. Ferreira, J. Liu, B. H. Krogh, N. Popli, and M. D. Ilic, "Wind integration in power systems: Operational challenges and possible solutions," Proc. IEEE, vol. 99, pp. 214-232, Jan. 2011.

[16] E. Lannoye, D. Flynn, and M. O'Malley, "Evaluation of power system flexibility," IEEE Trans. Power Syst., vol. 27, no. 2, pp. 922-931, 2012.

[17] R. Doherty and M. O'Malley, "A new approach to quantify reserve demand in systems with significant installed wind capacity," IEEE Trans. Power Syst., vol. 20, pp. 587595, May 2005.

[18] N. Troy, D. Flynn, and M. O'Malley, "The importance of sub-hourly modeling with a high penetration of wind generation," in Proc. 2012 IEEE Power and Energy Society General Meeting, (San Diego, CA), pp. 1-6.

[19] J. Ma, V. Silva, R. Belhomme, D. S. Kirschen, and L. F. Ochoa, "Evaluating and planning flexibility in sustainable power systems," IEEE Trans. Sustain. Energy, vol. 4, pp. 200-209, Jan. 2013.

[20] E. Ela and M. O'Malley, "Studying the variability and uncertainty impacts of variable generation at multiple timescales," IEEE Trans. Power Syst., vol. 27, pp. 1324-1333, Aug. 2012.

[21] North American Electric Reliability Corporation, "Special report. flexibility requirements and potential. metrics for variable generation: Implications for system planning studies," tech. rep., NERC, Princeton, NJ, 2010. 
[22] Y. Makarov, C. Loutan, and P. de Mello, "Operational impacts of wind generation on California power systems," IEEE Trans. Power Syst., vol. 24, pp. 1039-1050, May 2009.

[23] Y. Dvorkin, D. S. Kirschen, and M. A. Ortega-Vazquez, "Assessing flexibility requirements in power systems," IET Gener. Transm. Distrib., vol. 8, pp. 1820-1830, Nov. 2014.

[24] A. Ulbig and G. Andersson, "On operational flexibility in power systems," in Proc. 2012 IEEE Power and Energy Society General Meeting, (San Diego, CA), pp. 1-8.

[25] T. Zheng, J. Zhao, F. Zhao, and E. Litvinov, "Operational flexibility and system dispatch," in Proc. 2012 IEEE Power and Energy Society General Meeting, (San Diego, $\mathrm{CA})$.

[26] J. Apt, "The spectrum of power from wind turbines," J. Power Sources, vol. 169, no. 2, pp. 369-374, 2007.

[27] M. A. Ortega-Vazquez and D. S. Kirschen, "Should the spinning reserve procurement in systems with wind power generation be deterministic or probabilistic?," in Proc. 2009 Int. Conf. Sustainable Power Generation and Supply, (Nanjing, P. R. China), pp. 1-9.

[28] K. Hedman, R. O'Neill, E. Fisher, and S. Oren, "Optimal transmission switching sensitivity analysis and extensions," in Proc. 2009 IEEE Power Energy Society General Meeting, (Calgary, AB), pp. 1-8.

[29] E. Lannoye, D. Flynn, and M. O'Malley, "Transmission, variable generation, and power system flexibility," IEEE Trans. Power Syst., vol. 30, pp. 57-66, Jan. 2015.

[30] A. J. Lamadrid and T. Mount, "Ancillary services in systems with high penetrations of renewable energy sources, the case of ramping," Energy Economics, vol. 34, no. 6, pp. 1959-1971, 2012.

[31] J. Baker, "New technology and possible advances in energy storage," Energy Policy, vol. 36, no. 12, pp. 4368-4373, 2008.

[32] P. J. Hall and E. J. Bain, "Energy-storage technologies and electricity generation," Energy Policy, vol. 36, no. 12, pp. 4352-4355, 2008.

[33] C. Jin, J. Tang, and P. Ghosh, "Optimizing electric vehicle charging with energy storage in the electricity market," IEEE Trans. Smart Grid, vol. 4, pp. 311-320, March 2013. 
[34] R. Diao, S. Lu, M. Elizondo, E. Mayhorn, Y. Zhang, and N. Samaan, "Electric water heater modeling and control strategies for demand response," in Proc. 2012 IEEE Power and Energy Society General Meeting, (San Diego, CA), pp. 1-8.

[35] L. Soder, "Reserve margin planning in a wind-hydro-thermal power system," IEEE Trans. Power Syst., pp. 564-571, May 1993.

[36] F. D. Galiana, F. Bouffard, J. M. Arroyo, and J. F. Restrepo, "Scheduling and pricing of coupled energy and primary, secondary, and tertiary reserves," Proc. IEEE, vol. 93, pp. 1970-1983, Nov. 2005.

[37] European Network of Transmission System Operators for Electricity, "Supporting document for the network code on load-frequency control and reserves," tech. rep., ENTSO-E, Brussels, Belgium, 2013.

[38] F. Bouffard, F. D. Galiana, and A. Conejo, "Market-clearing with stochastic securityPart I: formulation," IEEE Trans. Power Syst., vol. 20, pp. 1818-1826, Nov. 2005.

[39] S. M. Chan, D. C. Powell, M. Yoshimura, and D. H. Curtice, "Operations requirements of utilites with wind power generation," IEEE Trans. Power App. Syst., vol. PAS-102, pp. 2850-2860, Sept. 1983.

[40] R. Doherty and M. O'Malley, "Quantifying reserve demands due to increasing wind power penetration," in Proc. 2003 IEEE PowerTech, vol. 2, (Bologna, Italy), pp. 1-5.

[41] M. A. Matos and R. J. Bessa, "Setting the operating reserve using probabilistic wind power forecasts," IEEE Trans. Power Syst., vol. 26, pp. 594-603, May 2011.

[42] M. A. Ortega-Vazquez and D. S. Kirschen, "Estimating the spinning reserve requirements in systems with significant wind power generation penetration," IEEE Trans. Power Syst., vol. 24, pp. 114-124, Feb. 2009.

[43] E. Lannoye, D. Flynn, and M. O'Malley, "Power system flexibility assessment - state of the art," in Proc. 2012 IEEE Power and Energy Society General Meeting, (San Diego, CA), pp. 1-6.

[44] C. De Jonghe, B. F. Hobbs, and R. Belmans, "Optimal generation mix with shortterm demand response and wind penetration," IEEE Trans. Power Syst., vol. 27, pp. 830-839, May 2012.

[45] E. Lannoye, D. Flynn, and M. O'Malley, "Assessment of power system flexibility: A high-level approach," in Proc. 2012 IEEE Power and Energy Society General Meeting, (San Diego, CA), pp. 1-8. 
[46] M. Schulze, L. Friedrich, and M. Gautschi, "Modeling and optimization of renewables: applying the energy hub approach," in Proc. 2008 IEEE Sustainable Energy Technologies, (Singapore), pp. 83-88.

[47] M. Schulze and P. C. Del Granado, "Optimization modeling in energy storage applied to a multi-carrier system," in Proc. 2010 IEEE Power and Energy Society General Meeting, (Minneapolis, MN), pp. 1-7.

[48] T. Krause, F. Kienzle, Y. Liu, and G. Andersson, "Modeling interconnected national energy systems using an energy hub approach," in Proc. 2011 IEEE PowerTech, (Trondheim, Norway).

[49] S. Pazouki, M. R. Haghifam, and J. Olamaei, "Economical scheduling of multi carrier energy systems integrating renewable, energy storage and demand response under energy hub approach," in Proc. 2013 IEEE Smart Grid Conference, (Tehran, Iran).

[50] Y. V. Makarov, P. V. Etingov, J. Ma, Z. Huang, and K. Subbarao, "Incorporating uncertainty of wind power generation forecast into power system operation, dispatch, and unit commitment procedures," IEEE Trans. Sustain. Energy, vol. 2, pp. 433-442, Oct. 2011.

[51] M. S. Nazir and F. Bouffard, "Intra-hour wind power characteristics for flexible operations," in Proc. 2012 IEEE Power and Energy Society General Meeting, (San Diego, CA), pp. 1-8.

[52] D. Lee and R. Baldick, "Analyzing the variability of wind power output through the power spectral density," in Proc. 2012 IEEE Power and Energy Society General Meeting, (San Diego, CA), pp. 1-8.

[53] W. Lin, J. Wen, S. Cheng, and W.-J. Lee, "An investigation on the active-power variations of wind farms," IEEE Trans. Ind. Appl., vol. 48, no. 3, pp. 1087-1094, 2012.

[54] M. A. Ortega-Vazquez and D. S. Kirschen, "Optimizing the spinning reserve requirements using a cost/benefit analysis," Power Syst. IEEE Trans., vol. 22, pp. 24-33, Feb. 2007.

[55] Y. Dvorkin, H. Pandzic, M. A. Ortega-Vazquez, and D. S. Kirschen, "A hybrid stochastic/interval approach to transmission-constrained unit commitment," IEEE Trans. Power Syst., pp. 1-11, 2014.

[56] L. Wu, M. Shahidehpour, and Z. Li, "Comparison of scenario-based and interval optimization approaches to stochastic SCUC," IEEE Trans. Power Syst., vol. 27, pp. 913921, May 2012. 
[57] J. M. Morales, A. J. Conejo, and J. Perez-Ruiz, "Economic valuation of reserves in power systems with high penetration of wind power," IEEE Trans. Power Syst., vol. 24, pp. 900-910, May 2009.

[58] E. Ela, M. Milligan, and B. Kirby, "Operating reserves and variable generation operating reserves and variable generation," tech. rep., Golden, Colorado, 2011.

[59] Bonneville Power Authority (BPA), "2014 total load \& wind generation in the BPA control area," http://transmission.bpa.gov/BUSINESS/OPERATIONS/WIND/.

[60] A. Bemporad, F. Borrelli, and M. Morari, "Min-max control of constrained uncertain discrete-time linear systems," IEEE Trans. Autom. Cont., vol. 48, pp. 1600-1606, Sept. 2003.

[61] W. Lin, J. Wen, S. Cheng, and W.-J. Lee, "An investigation on the active-power variations of wind farms," IEEE Trans. Ind. App., vol. 48, pp. 1087-1094, May 2012.

[62] M. Milligan, P. Donohoo, D. Lew, and E. Ela, "Operating reserves and wind power integration: an international comparison," in Proc. 2010 Int. Workshop on Large-Scale Integration of Wind Power into Power Systems, (Quebec, QC).

[63] P. Meibom, R. Barth, B. Hasche, H. Brand, C. Weber, and M. O'Malley, "Stochastic optimization model to study the operational impacts of high wind penetrations in Ireland," IEEE Trans. Power Syst., vol. 26, pp. 1367-1379, Aug 2011.

[64] G. Morales-España, A. Ramos, and J. Garcia-Gonzalez, "An MIP formulation for joint market-clearing of energy and reserves based on ramp scheduling," IEEE Trans. Power Syst., vol. 29, pp. 476-488, Jan 2014.

[65] K. W. Hyun, "From infinite horizon to receding horizon for controls, estimations and optimizations," in Proc. 2007 IEEE Chinese Control Conf., (Hunan, P. R. China), pp. $12-20$.

[66] C. Wang and S. M. Shahidehpour, "Ramp-rate limits in unit commitment and economic dispatch incorporating rotor fatigue effect," IEEE Trans. Power Syst., vol. 9, pp. 1539-1545, Aug 1994.

[67] I. T. Jolliffe, Principal Component Analysis. Springer Series in Statistics, Springer New York, 2006.

[68] J. Shlens, "A tutorial on principal component analysis," arXiv preprint arXiv:1404.1100, 2014. 
[69] B. Wang and B. F. Hobbs, "A flexible ramping product: Can it help real-time dispatch markets approach the stochastic dispatch ideal?," Elect. Power Syst. Res., vol. 109, pp. 128-140, 2014.

[70] T. Li and M. Shahidehpour, "Dynamic ramping in unit commitment," IEEE Trans. Power Syst., vol. 22, pp. 1379-1381, Aug 2007. 